UNIVERSIDADE DE SÃO PAULO

INSTITUTO DE FÍSICA

\title{
MODELOS ESTATÍSTICOS DE CAMPO MÉDIO PARA VIDROS DE SPINS E FLUIDOS COMPLEXOS
}

\author{
Danilo Barbosa Liarte
}

Tese de doutorado apresentada ao Instituto de Física da Universidade de São Paulo para obtenção do título de doutor em ciências.

Orientador: Prof. Dr. Carlos Seihiti Orii Yokoi

\section{BANCA EXAMINADORA}

Prof. Dr. Carlos Seihiti Orii Yokoi (IFUSP)

Prof. Dr. Antonio Martins Figueiredo Neto (IFUSP)

Profa. Dra. Vera Bohomoletz Henriques (IFUSP)

Prof. Dr. Jeferson Arenzon (UFRGS)

Prof. Dr. Jürgen Fritz Stilck (UFF)

São Paulo, SP

Julho de 2011 
FICHA CATALOGRAFICA

Preparada pelo Serviço de Biblioteca e Informação do Instituto de Física da Universidade de São Paulo

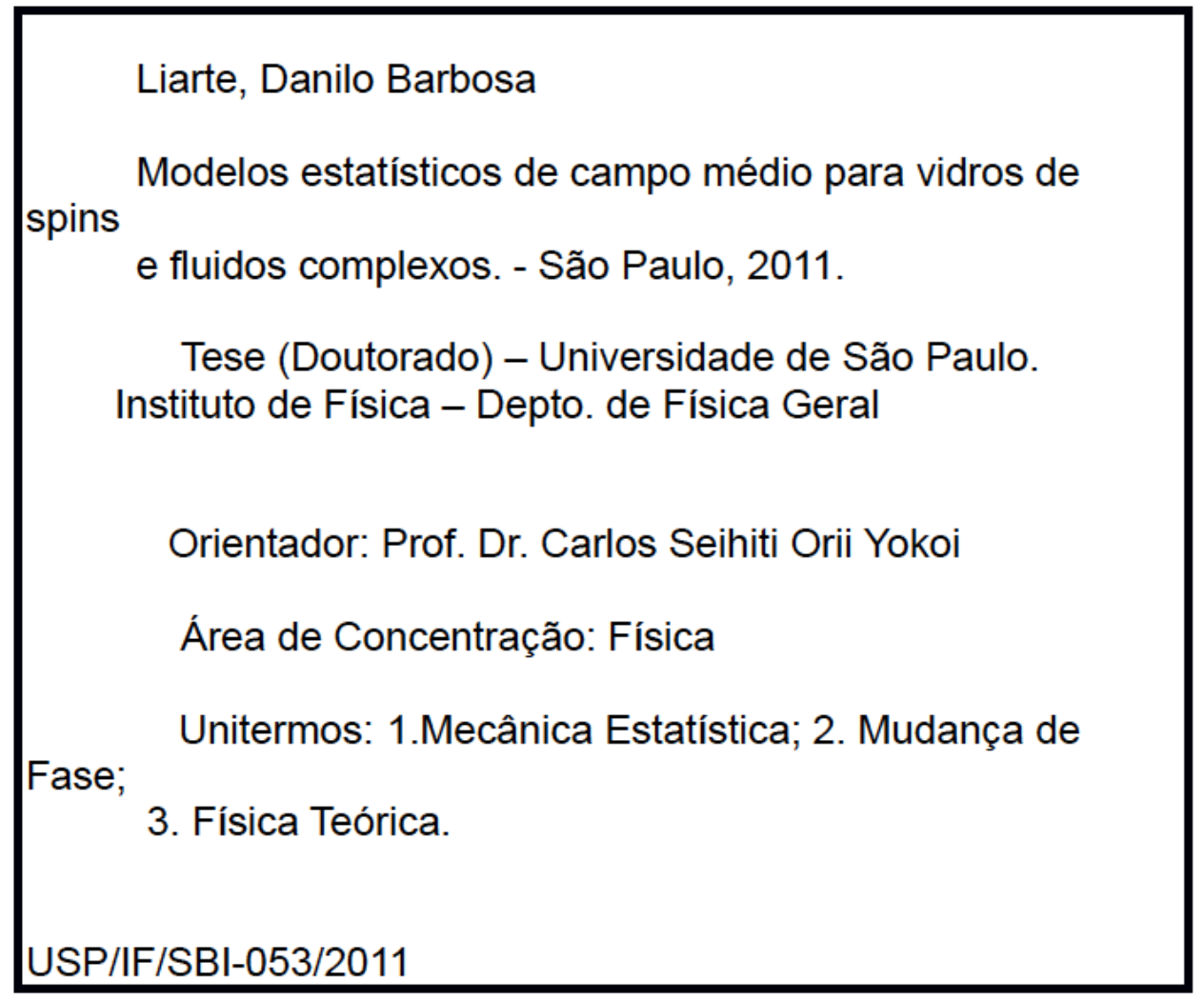


"The Scientist does not study nature because it is useful to do so. He studies it because he takes pleasure in it; and he takes pleasure in it because it is beautiful. If nature were not beautiful, it would not be worth knowing and life would not be worth living... I mean the intimate beauty which comes from the harmonious order of its parts and which a pure intelligence can grasp."

(Fonte: S. Chandrasekhar, "Beauty and the quest for beauty in science", Physics Today, December 2010. Tradução de um texto de Henri Poincaré, em "Science et méthode".) 



\section{Agradecimentos}

À minha família, em especial aos meus pais Antônio e Francisca, meus irmãos Sélia, Alan, Jardilson, Daniel e David, minha irmã na criação Francisca, meus tantos sobrinhos e minha querida noiva, Thais, pelo incomensurável apoio, carinho e exemplo, fontes de contínua inspiração.

Ao meu orientador, Prof. Carlos Yokoi, ao meu coorientador, Prof. Silvio Roberto Salinas, e ao Prof. José Pimentel de Lima, educadores formidáveis a quem devo orientação, supervisão, assim como tantas conversas estimulantes.

Aos amigos e colegas, dentro e fora da vida acadêmica.

A vários dos professores da Universidade Federal do Piauí e da Universidade de São Paulo, pelo auxílio na formação acadêmica.

Aos Profs. Carlos Eugênio, Tânia Tomé e Vera Henriques, pelas sugestões e dúvidas apresentadas no meu exame de qualificação.

Aos Profs. Antonio Figueiredo, Jeferson Arenzon, Jürgen Stilck e Vera Henriques, pelas sugestões e dúvidas apresentadas na minha defesa de tese de doutorado.

Ao Prof. Mário Tamashiro, membro suplente da comissão examinadora, pelas valiosas sugestões.

Aos amigos Alexei, Masayuki e Silas, com quem discuti e aprendi vários conceitos em matemática.

Ao $\mathrm{CNPq}$, pelo apoio financeiro. 



\section{Resumo}

Estudamos nesta tese três sistemas desordenados distintos das áreas de vidros de spins e fluidos complexos, por meio de modelos estatísticos no contexto da aproximação de campo médio. Analisamos os efeitos da inclusão de graus de liberdade elásticos sobre o diagrama de fases do modelo de Sherrington-Kirkpatrick, que é a versão de campo médio de um modelo popular de vidros de spins, paradigmas de sistemas com desordem temperada. Analisamos em seguida alguns problemas típicos da física dos fluidos complexos. Investigamos o diagrama de fases de um modelo de Maier-Saupe (MS), que é uma espécie de arquétipo das transições nemáticas, numa versão de rede muito simples, denominada modelo de Maier-Saupe-Zwanzig (MSZ), com a introdução de uma variável binária de desordem para representar uma mistura de discos e cilindros. Mostramos que o aparecimento de uma fase nemática biaxial, termodinamicamente estável, que tem sido intensamente procurada na literatura, depende da forma de tratamento das variáveis de desordem. Finalmente, utilizamos o modelo MSZ, na presença de termos elásticos não lineares e de elementos de desordem, a fim de reproduzir diversas características do comportamento termodinâmico dos elastômeros nemáticos, novos materiais poliméricos, com propriedades dos cristais líquidos nemáticos e das borrachas, tema de grande interesse na física da matéria mole. 



\section{Abstract}

We study three distinct disordered systems in the areas of spin glasses and complex fluids, by means of mean-field statistical models. We first analyze the effects of compressibility on the phase diagram of the Sherrington-Kirkpatrick model, a mean-field version of a popular model of spin glasses, which are paradigmatic examples of systems with quenched disorder. We then analyze some typical problems in the area of physics of complex fluids. We investigate the phase diagram of a Maier-Saupe model (MS), which is a sort of archetype of nematic transitions, in a simple lattice version called Maier-SaupeZwanzig model (MSZ), with the introduction of a binary variable of disorder to mimic a mixture of rod-like and plate-like mesogens. We show that the emergence of a stable nematic biaxial phase, which has been intensely pursued in the literature, depends on the form of treatment of the disorder variables. Finally, we use the MSZ model, in the presence of non-linear elastic terms and elements of disorder, to reproduce several aspects of the thermodynamic behavior of nematic elastomers, new polymeric materials with the properties of liquid crystals and rubber, and of great importance in the area of soft-matter physics. 



\section{Sumário}

$\begin{array}{ll}\text { Prefácio } & 1\end{array}$

1 Introdução 3

2 Modelo de Ising e métodos da teoria de campo médio $\quad 7$

2.1 Definição do modelo e revisão de resultados . . . . . . . . . . . . . 8

2.2 Teoria de campo médio . . . . . . . . . . . . . . . . . . . . . . . 11

2.3 Resultados termodinâmicos . . . . . . . . . . . . . . 16

2.4 Teoria de Landau, expoentes críticos e o critério de Ginzburg . . . . . . . . 22

2.5 Modelo de Ising com campos aleatórios . . . . . . . . . . . . . . . 25

3 Efeitos de compressibilidade no modelo de vidros de spins de Sherrington-

$\begin{array}{ll}\text { Kirkpatrick } & 29\end{array}$

3.1 O sistema: Vidros de spins . . . . . . . . . . . . . . . . . . . . . . 30

3.2 O problema físico . . . . . . . . . . . . . . . . . 34

3.3 Modelo de Sherrington-Kirkpatrick compressível . . . . . . . . . . . . 36

3.4 Método das réplicas . . . . . . . . . . . . . . . . . 37

3.5 Solução com simetria de réplicas e análise do comportamento crítico . . . . 40

3.6 Diagrama de fases e considerações finais . . . . . . . . . . . . . . . . . 44

4 Ordenamento nemático biaxial em misturas de discos e cilindros 49

4.1 Cristais líquidos nemáticos . . . . . . . . . . . . . . . 50

4.2 O problema físico . . . . . . . . . . . . . . . . 54

4.3 Formulação estatística em duas temperaturas . . . . . . . . . . . . 57 
4.4 Modelo de Maier-Saupe-Zwanzig desordenado . . . . . . . . . . . . . . . 61

4.5 Teoria de Landau-De Gennes . . . . . . . . . . . . . . . . . . . . 64

4.6 Considerações finais . . . . . . . . . . . . . . . . . . . . . . . 67

5 Transições de fases em elastômeros nemáticos $\quad 71$

5.1 Elastômeros e elastômeros nemáticos . . . . . . . . . . . . . . . . . 72

5.2 O problema físico . . . . . . . . . . . . . . . . . . 75

5.3 Modelo de rede para um elastômero nemático . . . . . . . . . . . . . . 78

5.4 Cálculo da energia livre . . . . . . . . . . . . . . . . . . . . . . 82

5.5 Resultados termodinâmicos . . . . . . . . . . . . . . . . . 86

5.5.1 Resultados na ausência de desordem $(\gamma=0) \ldots . . . . . .887$

5.5.2 Efeitos da desordem $(\gamma>0) \ldots \ldots . \ldots 88$

5.5.3 Resultados para desordem isotrópica $(c=1 / 3, \gamma>0) \ldots . . . .90$

5.6 Considerações finais . . . . . . . . . . . . . . . . . . . . . . . . 91

A Estabilidade da solução com simetria de réplicas $\quad 95$

B Solução trigonométrica da equação cúbica 99

$\begin{array}{ll}\text { Referências Bibliográficas } & 101\end{array}$ 


\section{Lista de Figuras}

2.1 Ernst Ising (à esquerda) e Wilhelm Lenz (à direita). Fonte: (Brush, 1967). 8

2.2 Ilustração de um sistema de spins do tipo Ising numa rede quadrada. Em geral, os íons magnéticos podem formar domínios de vários tamanhos, ou seja, conjuntos de spins vizinhos com o mesmo sinal (domínios negativos estão delimitados por linhas pontilhadas). Tais domínios possuem um papel importante na análise da criticalidade e comportamento de escala do sistema. $\quad 9$

$2.3 m \times \tanh (m / T)$ e $m \times m$ (gráfico à esquerda); $m \times g$ (gráfico à direita) para $T=1.25$ e $H=0 \ldots \ldots \ldots \ldots \ldots \ldots$. . . . . . . . . . . . . . . . 17

$2.4 m \times \tanh (m / T)$ e $m \times m$ (gráfico à esquerda); $m \times g$ (gráfico à direita) para $T=1$ e $H=0 \ldots \ldots \ldots \ldots \ldots \ldots$

$2.5 m \times \tanh (m / T)$ e $m \times m$ (gráfico à esquerda); $m \times g$ (gráfico à direita) para $T=0.75$ e $H=0$.

2.6 Parâmentro de ordem $m(\Gamma)$ como função da temperatura $T$ para o modelo de Ising a campo nulo na versão de Curie-Weiss (à esquerda). Os pontos do gráfico à direita são extraídos de medidas experimentais para o níquel, extraída de (Kittel, 1996). . . . . . . . . . . . . . 18

2.7 À esquerda: $m \times \tanh [(m+H) / T]$ e $m \times m$ para $H=0.05$ e $T=1.25 . \grave{A}$ direita: magnetização como função da temperatura para $H=0.05$. . . .

2.8 Magnetização versus campo magnético (gráfico à esquerda) e energia livre versus magnetização (gráfico à direita) para $T=1.25 \ldots$. . . . . . . . . . . 19

2.9 Magnetização versus campo magnético (gráfico à esquerda) e energia livre versus magnetização (gráfico à direita) para $T=1$. 
2.10 Magnetização versus campo magnético (gráfico à esquerda), magnetização corrigida versus campo magnético (gráfico interno à esquerda), energia livre versus magnetização (gráfico à direita) e energia livre versus campo

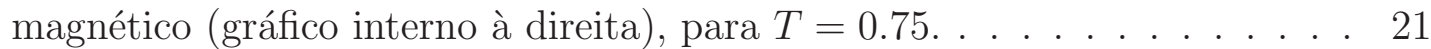

2.11 Diagrama de fases campo versus temperatura do modelo de Ising. Os termos Ferro e Para indicam ordenamento ferromagnético e paramagnético respectivamente. A linha $H=0$ no intervalo $(0,1)$ caracteriza uma transição de primeira ordem entre as fases ferromagnéticas positiva e negativa. $\mathrm{O}$ ponto $\mathrm{C}$ é o ponto crítico do sistema. . . . . . . . . . . . . . 22

2.12 Susceptibilidade magnética a campo nulo como função da temperatura para o modelo de Curie-Weiss.

3.1 Susceptibilidade magnética como uma função da temperatura, a campo nulo e não-nulo, para o AuFe. Extraído de (Cannella e Mydosh, 1972). . 31

3.2 Sir Samuel Edwards (foto à esquerda) e Philip Anderson (foto à direita). Fonte: www.wikipedia.com (maio de 2011) . . . . . . . . . . . . . . . . 32

3.3 Conjunto de variáveis (A, B e C) com interações frustradas. . . . . . . . . 33

3.4 Gráficos de $q^{1 / 2}$ e $m$ como função da temperatura para $a=1.5, b=0.5$, $j=1.75$ (à esquerda) e $j=2.25$ (à direita).

3.5 Diagrama de fases $J_{0} / J \times k_{B} T / J$ do modelo de Sherrington-Kirkpatrick compressível a campo nulo. . . . . . . . . . . . . . . . . . . . . . 45

3.6 Diagrama de fases $x \times T$ de um modelo de Sherrington-Kirkpatrick compressível a campo nulo em duas sub-redes.

4.1 a) Forma anisotrópica cilíndrica e discótica atribuída aos mesógenos. b) Ilustração de agregados moleculares anfifílicos presentes na composição de cristais líquidos liotrópicos. . . . . . . . . . . . . . . . . . 5 5

4.2 a) $\mathrm{O}$ ordenamento orientacional é favorecido tanto por interações estéricas de volume excluído quando interações de Van der Waals. b) Descrição do parâmetro de ordem nemático. c) Ilustração da transição entre as fases nemática e isotrópica em $T=T_{\mathrm{NI}} \ldots \ldots \ldots \ldots \ldots \ldots$ 
4.3 Ordenamento nemático uniaxial de cilindros (a), discos (b), e ordenamento nemático biaxial de misturas de discos e cilindros (c). . . . . . . . . . . 53

4.4 Diagrama de fases de uma mistura ternária de água deuterada, decanol e laurato de potássio. Fonte: (Yu e Saupe, 1980). . . . . . . . . . . . 55

4.5 Diagrama de fases temperatura versus concentração para misturas de discos e cilindros para variáveis de desordem temperadas (à esquerda), e recozidas (à direita). ISOTROPIC, BIAXIAL, $\mathrm{N}_{\mathrm{d}}$ e $\mathrm{N}_{\mathrm{c}}$ denotam as fases isotrópica, nemática biaxial, nemática uniaxial discótica e nemática uniaxial cilíndrica, respectivamente. Fonte: (Carmo et al., 2010). . . . . . . . . . . . . . . . 57

4.6 O tempo estimado para as variáveis associadas à diluição da rede alcançarem equilíbrio à temperatura $T_{f}$ é de aproximadamente $10^{-9} \sim 10^{-11} \mathrm{~s}$. De acordo com medidas de NMR e ESR, as variáveis de spins são termalizadas à temperatura $T_{s}$ entre $10^{-4}$ e $1 \mathrm{~s}$. As duas temperaturas precisam de minutos ou mesmo horas para se igualarem. A hipótese de duas temperaturas é, portanto, razoável para um regime onde $\tau_{f} \ll \tau_{s} \ll t_{1}$. . . . . . . . . 59

5.1 a) A aproximação da chama de uma vela a uma liga de borracha provoca uma contração na liga. b) Esboço de um possível motor térmico, alimentado por um desequilíbrio de massas gerado pelas contrações da borracha iluminada num único lado. Fonte: (Feynman, 1963). . . . . . . . . . . . . . 73

5.2 Representação de uma rede de polímeros com conexões cruzadas (à esquerda). No lado direito ilustramos uma única cadeia polimérica entre dois pontos de junção. . . . . . . . . . . . . . . . . . . . . .

5.3 Polímeros nemáticos com mesógenos anexados à cadeia principal (a); à cadeia lateral (b); à cadeia lateral em forma de T (c) . . . . . . . . . . . . 75

5.4 Comportamento de elastômeros nemáticos sujeitos a um aumento da tensão a temperatura fixa (a), e a uma diminuição da temperatura a tensão fixa

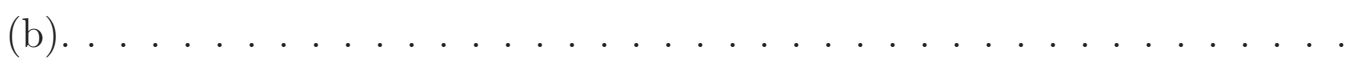

5.5 Parâmetro de ordem nemático $Q$ e anisotropia efetiva $r$ como função da temperatura reduzida $T / T_{\mathrm{NI}}$. Fonte: (Clarke et al., 2001). . . . . . . . . . 76 
5.6 Curvas experimentais para elastômeros nemáticos. (a) Tensão nominal como função da deformação. (b) Parâmetro de ordem nemático como função da tensão nominal. Fonte: (Fridrikh e Terentjev, 1999). . . . . . . . 78

5.7 Resultados experimentais para a tensão $\sigma$ e o parâmetro de ordem nemático $S$ como função da deformação $\epsilon$ para uma amostra formada no estado isotrópico (a) e nemático (b). Fonte: (Urayama et al., 2009). . . . . . . . . 78

5.8 Conexões cruzadas geram eixos de anisotropia (linhas tracejadas) que forçam um alinhamento dos diretores nemáticos locais em ambos os casos: (a) com um mesógeno fazendo a ponte entre duas cadeias poliméricas; (b) sem a presença de mesógenos no ponto de junção. . . . . . . . . . . . . . . . . . . 82

5.9 Parâmetro de ordem $S$ (curva inferior) e densidade de energia livre $f$ (curva superior) como funções da temperatura para $\gamma=0$ e tensão aplicada $\sigma=0.02 .87$

5.10 Parâmetro de ordem nemático (a) e fator de distorção (b) como função da temperatura, para $\gamma=0$ e vários valores da tensão aplicada $\sigma$. . . . . 88

5.11 Parâmetro de ordem nemático como uma função da temperatura para $\sigma=$ 0. (a) $\gamma=0.2$ e vários valores de $c$. (b) $c=0.4$ e vários valores de $\gamma . \quad$. . 89

5.12 A curva para $\sigma=0$ a direita da qual não existe transição de primeira ordem. A linha pontilhada vertical corresponde a $c=1 / 3 \ldots$. . . . . . . . 89

5.13 Fator de distorção $\lambda$ como uma função da temperatura para $\sigma=0, c=1 / 3$

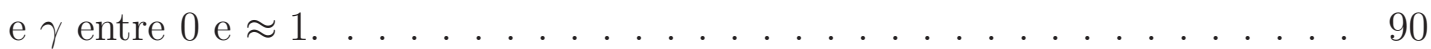

5.14 Curvas tensão versus deformação para $c=1 / 3$. (a) Isotermas para $\gamma=0.6$.

(b) Curvas iso- $\gamma$ para $T=0.8 \ldots \ldots \ldots$. . . . . . . . . . . . . . 91

5.15 Curvas tensão versus deformação para $T=0.95$. (a) $\gamma=0.45$ e $c=0.35$.

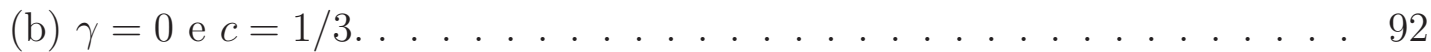




\section{Prefácio}

Parece-me que qualquer obra literária com mais de cem páginas merece uma explicação menos técnica sobre a organização e a estrutura do texto. A começar, gostaria que meu título não causasse a mesma impressão pedante que me causa o título do clássico de Per Bak: "How nature works". Minha tese é composta de três problemas que, embora fundamentalmente distintos, são abordados com o mesmo conjunto de métodos que se tornaram padrão na Física Estatística de sistemas desordenados. Condensar três trabalhos num único título, haja vista os objetivos não repousem sobre as técnicas, é uma tarefa

difícil, quando não impossível. É por essa mesma razão que escrevi uma introdução sucinta, talvez demasiamente geral, embora tenha me demorado no início de cada capítulo para discutir conceitos e motivações. Infelizmente, o leitor interessado em aspectos gerais terá que ler a introdução principal (Capítulo 1) e as introduções e motivações de cada trabalho nas seções iniciais dos Capítulos 3, 4 e 5.

Escrever números decimais em português tem sido uma fonte de problemas há vários anos. Embora meu lado nacionalista reconheça a importância de escrever em português correto, meu lado lógico me condena ao citar $T=2,5$ no texto e mostrar $T=2.5$ num gráfico. Modificar algumas dezenas de gráficos, produzidos em programas americanos, parece-me um trabalho "fora de propósito", o que me levou a usar pontos em todo o texto e esperar por uma absolvição dos mais críticos. Penso que outros problemas de escrita mais sérios podem ofuscar este detalhe. Por outro lado, tentei, sempre que possível, adaptar termos e expressões comuns em inglês, mas de ocorrência menos comum em português. O resultado poderá soar um desastre para os especialistas de cada tema, de forma que apreciaria qualquer recomendação nesse sentido. 
Ao terminar a redação, sete páginas de referências me pareceram um exagero mesmo para uma tese de quatro anos de doutoramento. Com tantas referências, minha intenção não se restringia a evitar mal-entendidos, como, por exemplo, deixar de dar os devidos créditos à fonte que deu origem a uma ideia ou resultado. Meu objetivo repousa antes numa forma de respeito e consideração aos autores. A esses, em especial aos que se dedicaram a escrever livros, que demandam mais trabalho, são menos influentes em critérios de avaliação, e provavelmente mais úteis, dedico uma frase de Henry David Thoreau, em "Walden":

"However much we may admire the orator's occasional bursts of eloquence, the noblest written words are commonly as far behind or above the fleeting spoken language as the firmament with its stars is behind the clouds. There are the stars, and they who can may read them. They are not exhalations like our daily colloquies and vaporous breath. What is called eloquence in the forum is commonly found to be rhetoric in the study. The orator yields to the inspiration of a transient occasion, and speaks to the mob before him, to those who can hear him; but the writer, whose more equable life is his occasion, and who would be distracted by the event and the crowd which inspire the orator, speaks to the intellect and heart of mankind, to all in any age who can understand him." 


\section{Capítulo 1}

\section{Introdução}

Roger Penrose, em seu livro "The road to reality", discute o conceito de um "universo platônico de formas matemáticas", constituído por um conjunto abstrato de regras de lógica, aparentemente independentes da mente particular de cada indivíduo, e relacionado ao nosso universo real por uma espécie de aproximação. Sob esse ponto de vista, o que chamamos leis fundamentais da física não são mais que modelos, habitantes do universo platônico de Penrose, de fenômenos do mundo real, aproximado. Nosso conhecimento e elegância de escrita (talvez ignorância) chegaram a tal ponto que essas leis podem ser condensadas numas poucas linhas, embora suas implicações, assim como a própria linguagem matemática em que são escritas, possam preencher um sem-número de bibliotecas. Permeando essas leis estão as teorias da Mecânica Estatística e da Termodinâmica. A Mecânica Estatística, em particular, tem como objetivo principal elucidar propriedades macroscópicas de sistemas de muitos corpos partindo de interações microscópicas fundamentais, valendo-se de ideias e conceitos de probabilidade e estatística, assim como das leis fundamentais da física. Infelizmente, uma abordagem direta, partindo dessas leis, quase nunca é factível, de forma que se torna necessário simplificar, reduzir, e remodelar os princípios básicos aos quais estamos habituados. Tal redução dá origem ao que chamamos modelos estatísticos, que compõem o esqueleto desta tese. Que o reducionismo inerente a esse tipo de tratamento não destrua o comportamento do sistema como um todo é questionável, mas deixemos de lado uma discussão mais filosófica em favor de uma abordagem mais prática e objetiva. 
Modelos estatísticos obtiveram sucesso impressionante na descrição de propriedades físicas de materiais nos estados sólido e gasoso. No estado gasoso, provavelmente devido à fraca interação entre átomos, moléculas, ou componentes do sistema ${ }^{1}$. No caso de sólidos, o sucesso se deve à estrutura cristalina sobre a qual átomos ou moléculas se organizam, caracterizada por simetria translacional. São as fortes interações e a ausência de simetria translacional que tornam a descrição teórica de líquidos tão difícil. Sistemas desordenados, em geral sólidos ou líquidos com impurezas, enfrentam problemas similares, presentes mesmo em abordagens numéricas, tornadas populares pelo considerável (ainda crescente) avanço da capacidade de processamento dos computadores atuais. O fato é que, sob o ponto de vista analítico, pouco se sabe sobre líquidos e sistemas desordenados além dos resultados obtidos por meio de aproximações efetivas, ou de campo médio.

Para descrever sistemas desordenados precisamos de, pelo menos, dois conjuntos de variáveis, que chamamos variáveis dinâmicas e variáveis de desordem. Variáveis dinâmicas descrevem estados estatísticos nos ensembles de Gibbs, como spins de um sistema magnético. Variáveis de desordem podem ser classificadas em dois grupos, de acordo com a relação entre seus tempos de relaxação e os das variáveis dinâmicas associadas. Variáveis dinâmicas em sistemas cuja desordem é temperada (quenched) relaxam de forma muito mais rápida que as variáveis aleatórias de desordem. Nesses sistemas, a energia necessária para alterar uma configuração de desordem é muito maior do que $k T_{c}$, onde $k$ é a constante de Boltzmann e $T_{c}$ é a temperatura de transição de fase (Thorpe, 1978), de forma que a desordem pode ser considerada fixa para escalas de tempo factíveis experimentalmente. Por outro lado, em sistemas de desordem recozida (annealed) os tempos de relaxação das variáveis dinâmicas e de desordem são comparáveis. Neste caso, ao contrário do caso temperado, a desordem não é fixa, mas evolui, para cada temperatura, até que o sistema atinja a configuração de menor energia livre. Alguns sistemas podem ainda ser descritos tanto por variáveis temperadas quanto recozidas, ou mesmo estar localizados numa situação intermediária, como acontece a alguns polímeros heterogêneos quimicamente desordenados (Patel e Fredrickson, 2003), ou superfícies desordenadas de macromoléculas carregadas (Mamasakhlisov, Naji, e Podgornik, 2008).

\footnotetext{
${ }^{1}$ A descrição de sistemas gasosos com interações de longo alcance permanece um considerável desafio.
} 
Estudamos nessa tese três sistemas desordenados distintos, por meio de modelos estatísticos, no contexto da aproximação de campo médio. Nosso interesse repousará principalmente sobre as transições de fases e fenômenos críticos desses sistemas, que são provavelmente o objeto de estudo mais intenso na área de Mecânica Estatística. Para introduzir conceitos essenciais da teoria de campo médio, apresentamos no Capítulo 2 uma revisão de alguns resultados do modelo de Ising, na versão de Curie-Weiss. No primeiro problema, apresentado no Capítulo 3 e resumidamente em (Liarte, Salinas, e Yokoi, 2009), analisamos os efeitos da compressibilidade sobre o diagrama de fases do modelo de Sherrington-Kirkpatrick, que é a versão de campo médio de um modelo popular de vidros de spins, arquétipos de sistemas com desordem temperada. Analisamos em seguida alguns problemas típicos da física dos fluidos complexos. Investigamos, no Capítulo 4 e na referência (Carmo, Liarte, e Salinas, 2010), o diagrama de fases de um modelo de Maier-Saupe (MS), que é uma espécie de arquétipo das transições nemáticas, numa versão de rede muito simples, denominada modelo de Maier, Saupe e Zwanzig (MSZ), com a introdução de uma variável binária de desordem a fim de simular uma mistura de discos e cilindros. Mostramos que o aparecimento de uma fase nemática biaxial, termodinamicamente estável, que tem sido intensamente procurada na literatura, depende da forma de tratamento das variáveis de desordem. Finalmente, utilizamos o modelo MSZ, na presença de termos elásticos não lineares e de elementos de desordem, a fim de reproduzir diversas características do comportamento termodinâmico dos elastômeros nemáticos, novos materiais poliméricos, com propriedades dos cristais líquidos nemáticos e das borrachas, tema de grande interesse na física da matéria mole. Esses resultados são apresentados no Capítulo 5 e resumidamente em (Liarte, Salinas, e Yokoi, 2011). 



\section{Capítulo 2}

\section{Modelo de Ising e métodos da teoria}

\section{de campo médio}

O modelo de Ising, ou modelo de Lenz-Ising ${ }^{1}$, possui grande parcela de responsabilidade pelo nível de desenvolvimento atingido na área de modelagem na Mecânica Estatística do século XX. Tanta popularidade decorre, provavelmente, de sua universalidade. De fato, um sem-número de artigos é publicado diariamente onde a simetria do modelo de Ising é incorporada a modelos para os mais diversos sistemas. Talvez a analogia mais conhecida seja com o gás de rede proposto por T. D. Lee e C. N. Yang para descrever a transição entre as fases líquida e gasosa de um fluido simples (Lee e Yang, 1952). Outros exemplos em que a energia de interação é uma variante da energia de Ising vão desde os chamados vidros de spins (Sherrington e Kirkpatrick, 1975) a alguns sistemas de interesse biológico (Reis, 2011). Não obstante a ausência de uma solução analítica exata para o modelo em três dimensões ${ }^{2}$, é relativamente simples obter resultados aproximados que forneçam um bom panorama qualitativo dos sistemas modelados por tais interações. É com essa perspectiva que utilizaremos o modelo de Ising como laboratório para introduzir alguns métodos e análises da teoria de campo médio. Vários cálculos desta tese são variações ou adaptações de cálculos bem-conhecidos, como os apresentados neste capítulo. Na seção

\footnotetext{
${ }^{1} \mathrm{O}$ modelo foi sugerido para descrever transições ferromagnéticas em 1920 por Wilhelm Lenz, dois anos antes de se tornar orientador de Ernst Ising (Figura 2.1), cuja dissertação está resumida num artigo publicado em 1925 (Brush, 1967).

${ }^{2} \mathrm{O}$ modelo bidimensional com a adição de um termo de interação com um campo magnético uniforme também não possui solução exata.
} 
2.1, definiremos o modelo e revisaremos alguns resultados principais para dimensão finita ${ }^{3}$. Na seção 2.2 abordaremos a teoria de campo médio, em especial a versão de Curie-Weiss para o modelo de Ising. Alguns resultados termodinâmicos são apresentados na seção 2.3. Uma discussão sobre fenomenologia de Landau, expoentes críticos e correções para o campo médio é apresentada na seção 2.4. Por fim, na seção 2.5 introduzimos desordem por meio do modelo de Ising com campos aleatórios.
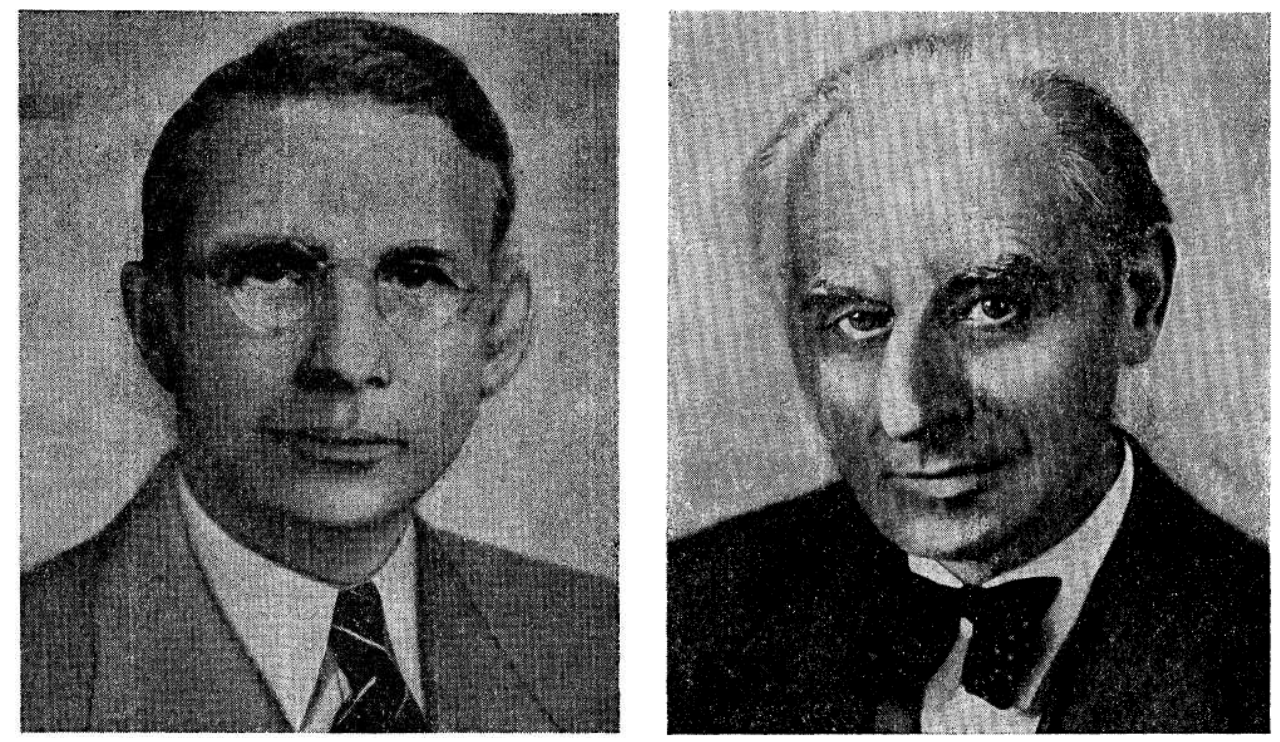

Figura 2.1: Ernst Ising (à esquerda) e Wilhelm Lenz (à direita). Fonte: (Brush, 1967).

\subsection{Definição do modelo e revisão de resultados}

Para extrairmos sentido da palavra simetria, enfatizada no primeiro parágrafo deste capítulo, precisamos discutir o que entendemos por modelo de Ising. Considere uma coleção de $N$ momentos magnéticos, como spins eletrônicos, distribuídos numa rede cristalina. Se as interações forem anisotrópicas, tal que apenas a interação das componentes dos spins numa dada direção seja relevante, podemos descrever a configuração de cada sítio da rede por uma variável binária, podendo assumir os valores +1 ou -1 em unidades apropriadas $^{4}$. Uma configuração de um tal sistema numa rede quadrada está ilustrada na

\footnotetext{
${ }^{3}$ Usamos o termo dimensão finita para refletir também o caráter de alcance finito (short-range) das interações, em contraste com a teoria de alcance infinito (infinite-range) de Curie-Weiss.

${ }^{4}$ Utilizaremos up e down, ou $+\mathrm{e}-$ com os mesmos significados.
} 
Figura 2.2. O modelo de Ising pode ser definido pela hamiltoniana

$$
\mathcal{H}=-J \sum_{(i, j)} \sigma_{i} \sigma_{j}-H \sum_{i=1}^{N} \sigma_{i},
$$

onde $J>0$ é um termo de interação de troca que favorece alinhamento paralelo entre os spins $\sigma_{i}= \pm 1,1 \leq i \leq N, H$ representa o campo magnético externo ${ }^{5}$, e a primeira soma em (2.1) deve ser restrita a pares de sítios vizinhos (contados uma vez).

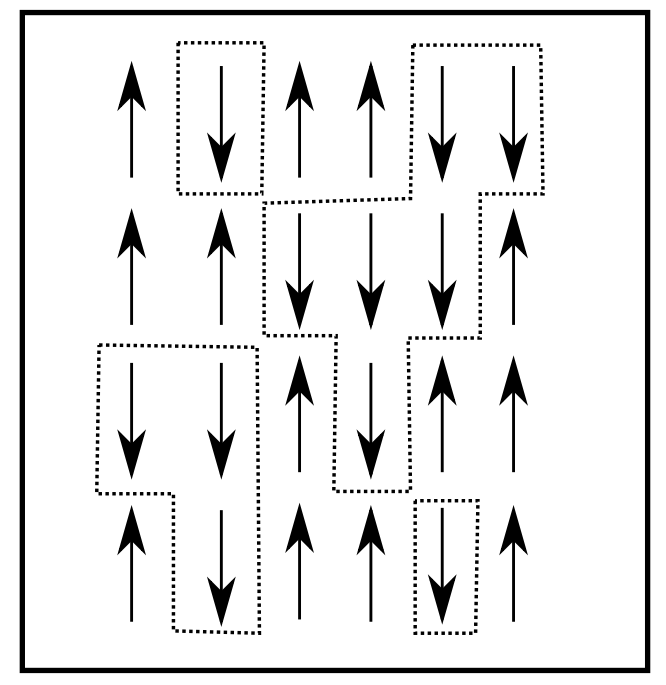

Figura 2.2: Ilustração de um sistema de spins do tipo Ising numa rede quadrada. Em geral, os íons magnéticos podem formar domínios de vários tamanhos, ou seja, conjuntos de spins vizinhos com o mesmo sinal (domínios negativos estão delimitados por linhas pontilhadas). Tais domínios possuem um papel importante na análise da criticalidade e comportamento de escala do sistema.

A hamiltoniana $\mathcal{H}$ dada por (2.1) é composta de dois termos. O primeiro, relacionado à interação intermolecular dentro do magneto, satisfaz a propriedade

$$
\mathcal{H}_{0}\left(\sigma_{1}, \cdots, \sigma_{N}\right)=\mathcal{H}_{0}\left(-\sigma_{1}, \cdots,-\sigma_{N}\right)
$$

decorrente da simetria por uma inversão temporal, que em última análise implica que $\mathcal{H}$ é invariante se os sinais dos campos e momentos magnéticos forem invertidos. Um modelo descrito por variáveis binárias e com interações com a simetria mencionada acima é chamado modelo de Ising, em geral, independente da forma explícita (2.1).

\footnotetext{
${ }^{5}$ Note que em (2.1) o campo magnético tem unidade de energia. É comum encontrar a expressão $-g \mu_{0} H \sigma_{z}$ para a energia de interação com o campo, onde $g$ é o fator de forma do átomo e $\mu_{0}$ é uma unidade padrão de momento magnético (geralmente o magneton de Bohr).
} 
Temperatura e campo magnético são grandezas facilmente controladas no laboratório, o que torna a energia livre $G=U-T S$ o potencial termodinâmico adequado para descrever magnetos simples, onde $U, T$ e $S$ são a energia interna, temperatura e entropia, respectivamente. O estado de equilíbrio, relacionado a um mínimo de $G$, deve emergir da competição entre as contribuições da energia e da entropia. A campo nulo e baixas temperaturas, o termo de energia é dominante, de forma que o estado de equilíbrio é caracterizado por um alinhamento paralelo entre os spins. Por outro lado, como a entropia é uma função monótona crescente da temperatura ${ }^{6}$, espera-se que sua contribuição se torne dominante para valores altos de $T$. Pode-se sugerir então que haja uma temperatura crítica $T=T_{c}$, separando uma fase ordenada, com magnetização $m>0$ (ferromagnética), a baixas temperaturas, de uma fase desordenada, com $m=0$ (paramagnética), a altas temperaturas ${ }^{7}$.

É importante questionar sobre a utilidade do modelo de Ising na descrição de materiais reais, haja vista que os momentos magnéticos dos elétrons podem, em geral, estar orientados ao longo de qualquer direção espacial. Um contato direto com experimentos só pode ser justificado para materiais com alto grau de anisotropia na interação entre os spins. Esse parece ser o caso do cloreto de ferro $\left(\mathrm{FeCl}_{2}\right)$ e do carbonato de ferro $\left(\mathrm{FeCO}_{3}\right)$, por exemplo ${ }^{8}$. Quando tal condição é satisfeita, espera-se que as previsões quantitativas do modelo de Ising em três dimensões para os expoentes críticos desses materiais sejam verificadas experimentalmente.

Ising de fato resolveu o modelo em uma dimensão espacial, mas errou ao prever por meio de argumentos heurísticos que não haveria transição de fases para dimensão $d=3$ (Brush, 1967). Soluções exatas para $d>1$ continuaram um desafio a físicos e matemáticos por quase 20 anos, quando Lars Onsager, um químico, anunciou sua solução do modelo de Ising bidimensional numa conferência da Academia de Ciências de Nova York, em 1942. Os detalhes foram publicados dois anos depois no periódico Physical Review (Onsager,

\footnotetext{
${ }^{6}$ Como deve ser, admitindo que as leis da termodinâmica de equilíbrio sejam válidas.

${ }^{7}$ A existência de uma temperatura crítica finita $\left(T_{c}>0\right)$ não é óbvia. O modelo de Ising em uma dimensão, por exemplo,

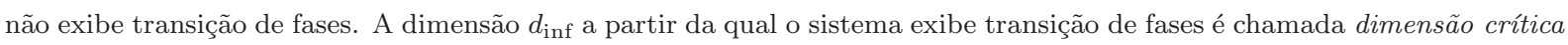
inferior do modelo. Para o modelo de Ising $d_{\text {inf }}=2$.

${ }^{8}$ Veja o capítulo 1, página 15 , de (Baxter, 1989) para referências.
} 
1944). Atualmente, cerca de 70 anos após o anúncio de Onsager, o modelo de Ising bidimensional com campo ou o modelo para $d=3$ continuam um cálice sagrado para vários físicos, suscitando discussões ante declarações audaciosas na literatura ${ }^{9}$.

Face a uma escassez de resultados exatos, expansões a baixas e altas temperaturas talvez sejam as melhores aproximações adotadas em estudos analíticos do modelo de Ising (Baxter, 1989). Trabalhos recentes fornecem resultados quantitativamente relevantes mesmo em regiões muito próximas ao ponto crítico. Por outro lado, um enorme volume de trabalho é necessário na obtenção dessas séries. É possível que o conjunto de conceitos e métodos reunidos sob o termo grupos de renormalização forneça a melhor relação custobenefício para obtenção analítica das propriedades críticas de modelos estatísticos. Vale notar, porém, que grupos de renormalização não constituem uma "caixa preta" onde se processa uma hamiltoniana e se colhem expoentes críticos como resultados. Exceto nos casos mais simples, é preciso adaptar as técnicas, e mesmo recorrer a uma série de aproximações com pouco ou nenhum controle para tratar as transformações de bloco envolvidas. Isso ocorre mesmo segundo o procedimento sistemático da expansão em $\epsilon$. Abordaremos na próxima seção um outro tipo de aproximação, talvez a mais popular, comumente denominada aproximação de campo médio. Essa classe de aproximações é relativamente simples, e fornece um bom panorama qualitativo do sistema abordado, embora em geral falhe na região crítica ${ }^{10}$.

\subsection{Teoria de campo médio}

Em favor de teorias de campo médio, Leo Kadanoff escreve que "precisão qualitativa é frequentemente boa o suficiente para várias aplicações úteis" (Kadanoff, 2009). Em alguns casos, como na descrição de supercondutores do tipo I (Cardy, 1996) e na aproximação de potencial coerente para vidros elásticos (He e Thorpe, 1985), mesmo concordância quantitativa pode ser observada. Quando reunida a uma análise dos resultados de grupos de renormalização, teorias de campo médio podem fornecer informações sobre a natureza

\footnotetext{
${ }^{9}$ Veja, por exemplo o artigo de Zhang (Zhang, 2007) e comentários posteriores (Wu, McCoy, Fisher, e Chayes, 2008).

${ }^{10} \mathrm{Um}$ outro tipo de abordagem teórica, baseado em simulações numéricas, tem desempenhado um papel cada vez mais importante na descrição de transições de fases e fenômenos críticos.
} 
das fases separadas por uma transição, em regiões do diagrama de fases distantes do ponto crítico, onde sua aplicabilidade é garantida. Tais informações não podem ser extraídas de um estudo de grupos de renormalização apenas.

De volta ao modelo de Ising, o problema estatístico reside no cálculo da função de partição

$$
Z=\operatorname{Tr} \exp \left(\beta J \sum_{(i, j)} \sigma_{i} \sigma_{j}+\beta H \sum_{i=1}^{N} \sigma_{i}\right)
$$

onde $\beta=1 / k T$ como usual, $T$ é a temperatura e $k$ é a constante de Boltzmann. A notação Tr indica uma soma sobre todos os valores dos spins $\sigma_{i}$. Note que $Z$ é uma função analítica da temperatura para $N$ finito. Assim, é interessante ressaltar que transições de fases, sendo descritas teoricamente por divergências de alguma derivada da energia livre associadas a regiões onde $Z$ não é analítica, devem estar intimamente ligadas ao limite termodinâmico.

O cálculo de $Z$ em (2.3) é trivial na ausência de interação entre os spins $(J=0)$, condição mais adequada na descrição de um paramagneto. A função de partição é o produto de $N$ funções de partição de uma partícula

$$
Z_{1}=\sum_{\sigma= \pm 1} \exp (\beta H \sigma)=2 \cosh (\beta H)
$$

e a magnetização $m$ é dada por

$$
m=\langle\sigma\rangle=\frac{\sum_{\sigma= \pm 1} \sigma e^{\beta H \sigma}}{\sum_{\sigma= \pm 1} e^{\beta H \sigma}}=\tanh (\beta H),
$$

onde $\langle\cdots\rangle$ denota uma média térmica usual. Para $J \neq 0$ o cálculo é muito mais complicado. Uma abordagem simples consistiria em linearizar a interação entre os spins sem destruir a ideia física principal. Isto é, em materiais ferromagnéticos, a temperaturas suficientemente baixas, deve haver magnetização não nula mesmo quando $H \rightarrow 0$.

O termo ubíquo aproximação de campo médio enquadra um sem-número de métodos distintos. Entre eles, uma maneira simples que expõe o caráter das aproximações envolvidas consiste em desprezar termos de segunda ordem em $\delta \sigma=\sigma-m$ na identidade (Cardy, 1996)

$$
\sigma_{i} \sigma_{j}=\left[m+\left(\sigma_{i}-m\right)\right]\left[m+\left(\sigma_{j}-m\right)\right]
$$


A função de partição fica dada então por

$$
\begin{aligned}
Z & =\operatorname{Tr} \exp \left(\beta J \sum_{(i, j)}\left[m+\left(\sigma_{i}-m\right)\right]\left[m+\left(\sigma_{j}-m\right)\right]+\beta H \sum_{i=1}^{N} \sigma_{i}\right) \\
& \approx \operatorname{Tr} \exp \left(-\frac{N \beta J^{\prime}}{2} m^{2}+\beta\left(J^{\prime} m+H\right) \sum_{i=1}^{N} \sigma_{i}\right),
\end{aligned}
$$

onde $J^{\prime}=z J$, sendo $z$ a coordenação da rede, isto é, o número de sítios vizinhos mais próximos. O termo $\delta \sigma \delta \sigma^{\prime}$ está relacionado a correlações espaciais entre spins vizinhos mais próximos, e deve possuir, em geral, importância fundamental na vizinhança do ponto crítico. Apenas em modelos onde o comprimento de correlação ${ }^{11}$ é suficientemente pequeno podemos esperar algum tipo de concordância quantitativa. Por outro lado, o problema agora é factível, uma vez que a energia efetiva proveniente dessa aproximação não possui interação entre os spins. Ao invés de perseguir esses resultados agora, tendo exposto o caráter das aproximações envolvidas e as ideias principais do método, vamos nos concentrar num outro tipo de abordagem, também de campo médio, para resolver o modelo de Ising. Para isso introduziremos o chamado modelo de Curie-Weiss (Salinas e Wreszinski, 1993; Salinas, 1997). Tal modelo pode ser obtido a partir de uma deformação do modelo de Ising, estendendo as interações de curto alcance a interações de alcance infinito, independentes da distância entre sítios, onde o parâmetro de troca deve escalar adequadamente com o tamanho do sistema (número de partículas) de forma a assegurar o limite termodinâmico ${ }^{12}$. Apenas após obter os resultados é possível verificar que essa abordagem reproduz os resultados de campo médio. A opção por estudar o modelo de Ising por meio do modelo de Curie-Weiss repousa no fato de que os cálculos, nesse caso,

\footnotetext{
${ }^{11} \mathrm{~A}$ correlação $g_{i j}$ entre os spins dos sítios $i$ e $j$ é definida por

$$
g_{i j}=\left\langle\sigma_{i} \sigma_{j}\right\rangle-\left\langle\sigma_{i}\right\rangle\left\langle\sigma_{j}\right\rangle
$$

Para hamiltonianas invariantes translacionais $g_{i j}$ deve depender apenas da distância $\boldsymbol{r}_{i j}$ entre os sítios $i$ e $j$, ou seja, $g_{i j}=g\left(\boldsymbol{r}_{i j}\right)$ onde $g(\boldsymbol{r})$ é a função de correlação. Longe do ponto crítico, $g(\boldsymbol{r})$ deve decair exponencialmente a zero para $r \gg 1$, segundo a forma

$$
g(x \boldsymbol{k}) \sim x^{-\tau} e^{-x / \xi}, \quad \text { quando } x \rightarrow \infty
$$

onde $\boldsymbol{k}$ é um vetor unitário fixo, $\tau$ é um número e $\xi$ é o comprimento de correlação na direção $\boldsymbol{k}$. A divergência do comprimento de correlação pode ser usada para caracterizar, de fato, um ponto crítico (Baxter, 1989).

${ }^{12}$ Em sistemas mais complicados, a dependência adequada do parâmetro de troca em $N$ só pode ser verificada ao final dos cálculos.
} 
são exatos, ou seja, não envolvem aproximações ${ }^{13}$.

O modelo de Curie-Weiss é definido pela hamiltoniana

$$
\mathcal{H}=-\frac{J}{N} \sum_{1 \leq i<j \leq N} \sigma_{i} \sigma_{j}-H \sum_{i=1}^{N} \sigma_{i}
$$

ou ainda,

$$
\begin{aligned}
\mathcal{H} & =-\frac{J}{2 N} \sum_{i, j=1}^{N} \sigma_{i} \sigma_{j}+\frac{J}{N} \sum_{i=1}^{N} \sigma_{i}^{2}-H \sum_{i=1}^{N} \sigma_{i} \\
& =-\frac{J}{2 N}\left(\sum_{i=1}^{N} \sigma_{i}\right)^{2}-H \sum_{i=1}^{N} \sigma_{i}+\mathrm{O}(1) .
\end{aligned}
$$

A contribuição de termos de ordem menor do que $N$ na hamiltoniana é nula no limite termodinâmico $(N \rightarrow \infty)$, de forma que omitiremos $\mathrm{O}(1)$ daqui por diante. Omitiremos também o intervalo das somas sobre as partículas onde não houver possibilidade de confusão. É possível entender a escolha da escala com $N$ das interações entre os spins observando que, a temperaturas muito baixas, os spins se encontram no estado ferromagnético, com quase todos os spins alinhados paralelamente. Tomando $\sigma_{i}=+1$ para todo $i$, verifica-se que o parâmetro de troca deve escalar com $1 / N$ para que a energia interna, associada a uma média da hamiltoniana, seja extensiva. A função de partição é dada por

$$
Z=\operatorname{Tr} \exp \left[\frac{\beta J}{2 N}\left(\sum_{i=1}^{N} \sigma_{i}\right)^{2}+\beta H \sum_{i=1}^{N} \sigma_{i}\right] .
$$

O termo quadrático em (2.12) pode ser linearizado por meio da identidade gaussiana

$$
\exp \left(\lambda a^{2}\right)=\sqrt{\frac{N}{2 \pi}} \int_{-\infty}^{+\infty} d x \exp \left(-\frac{1}{2} N x^{2}+\sqrt{2 N \lambda} a x\right),
$$

$\operatorname{com} a=\sum_{i} \sigma_{i}, \lambda=\beta J / 2 N$ e $x=\sqrt{\beta J} y$. Obtemos então

$$
Z=\sqrt{\frac{\beta J N}{2 \pi}} \int_{-\infty}^{+\infty} d y \exp \left(-\frac{1}{2} N \beta J y^{2}\right) \operatorname{Tr} \exp \left[\beta(J y+H) \sum_{i} \sigma_{i}\right],
$$

que é semelhante à equação (2.7), com a vantagem de não envolver nenhuma aproximação. Podemos agora usar o resultado (2.4) com $H \rightarrow J y+H$ para escrever a soma sobre os

\footnotetext{
${ }^{13}$ É importante fazer uma distinção semântica entre as palavras exato e rigoroso. Exato, significando não aproximado, nesse contexto, contrasta com rigoroso, relacionado ao rigor matemático.
} 
estados dos spins. Assim,

$$
\begin{aligned}
Z & =\sqrt{\frac{\beta J N}{2 \pi}} \int_{-\infty}^{+\infty} d y \exp \left(-\frac{1}{2} N \beta J y^{2}\right)\{2 \cosh [\beta(J y+H)]\}^{N} \\
& =\sqrt{\frac{\beta J N}{2 \pi}} \int_{-\infty}^{+\infty} d y \exp \left\{-\frac{1}{2} N \beta J y^{2}+N \ln 2 \cosh [\beta(J y+H)]\right\} .
\end{aligned}
$$

O argumento da integral em (2.15) é do tipo $\exp [-N \psi(y)]$, onde

$$
\psi(y)=\beta J y^{2} / 2-\ln 2 \cosh \beta(J y+H) .
$$

É possível calcular a integral acima por meio do método de integração assintótica de Laplace (Bruijn, 1981). A ideia é que, para $N$ grande, o valor da integral é dominado pelo valor máximo do integrando, ou seja, pelo ponto onde $\psi(y)$ é mínimo. Logo, para $N \gg 1$

$$
Z \approx \exp \left[-N \psi\left(y^{*}\right)\right]
$$

onde $y^{*}$ é escolhido tal que

$$
\left.\frac{d \psi}{d y}\right|_{y=y^{*}}=0,
$$

ou seja,

$$
y^{*}=\tanh \beta\left(J y^{*}+H\right) .
$$

A energia livre ${ }^{14}$ é dada por

$$
\begin{aligned}
g(T, H) & =-k T \lim _{N \rightarrow \infty} \frac{1}{N} \ln Z=k T \psi\left(y^{*}\right) \\
& =\frac{1}{2} J y^{* 2}-k T \ln 2 \cosh \beta\left(J y^{*}+H\right),
\end{aligned}
$$

onde $y^{*}$ satisfaz a equação (2.19). As equações (2.19) e (2.20) são exatamente as equações que obteríamos se tivéssemos continuado a análise da equação (2.7). Ou seja, observamos a posteriori que a abordagem de Curie-Weiss é do tipo campo médio, desde que $y^{*}$, introduzido numa manipulação matemática, corresponda, de fato, à magnetização. Por uma análise termodinâmica apenas, a magnetização $m$ deve ser dada por

$$
\begin{aligned}
m & =-\frac{\partial g}{\partial y^{*}} \frac{\partial y^{*}}{\partial H}-\frac{\partial g}{\partial H} \\
& =k T \beta \tanh \beta\left(J y^{*}+H\right)=y^{*},
\end{aligned}
$$

\footnotetext{
${ }^{14}$ A nomenclatura $g(T, H)$ para a energia livre magnética é apropriada dada a analogia com a função de Gibbs de um fluido puro, que depende de $p$ e $T$. Isto decorre devido à forma que escolhemos para a hamiltoniana (Salinas, 1997).
} 
onde usamos a equação (2.19) em ambas as linhas de (2.21).

\subsection{Resultados termodinâmicos}

Para simplificar a análise dos resultados, vamos escrever as energias ${ }^{15}$ em unidades de $J$ e a temperatura em unidades de $J / k$. As equações (2.19) e (2.20) ficam dadas por

$$
\begin{gathered}
m=\tanh \left(\frac{m+H}{T}\right), \\
g=\frac{1}{2} m^{2}-T \ln 2 \cosh \left(\frac{m+H}{T}\right) .
\end{gathered}
$$

A campo magnético nulo $(H=0)$, a equação $(2.22)$ pode ter até três soluções para $m$. Apresentamos gráficos de $m \times m$ e de $m \times \tanh (m / T)$ para $T=1.25, T=1$ e $T=0.75$ no lado esquerdo das figuras 2.3, 2.4 e 2.5, respectivamente. A energia livre (2.23) aparece no lado direito das mesmas figuras. Note que $g(m)$ só tem sentido do ponto de vista de equilíbrio termodinâmico no ponto de mínimo. Para $T=1.25$ (Figura 2.3), a equação (2.22) possui apenas uma solução, correspondente a magnetização nula no ponto A (gráfico à esquerda), onde a energia livre apresenta um mínimo (gráfico à direita). Para $T=1$ (Figura 2.4), os gráficos permanecem semelhantes aos do caso anterior, com a diferença que a curva $\tanh (m / T)$ é tangente à curva $m$ em $m=0$ e a energia livre se torna "achatada" na vizinhança deste ponto. Para $T=0.75$ (Figura 2.5), existem três soluções para a equação (2.22), correspondentes ao ponto A (magnetização nula), $\Gamma$ e $\Gamma^{*}$ (magnetização não nula), sendo $m\left(\Gamma^{*}\right)=-m(\Gamma)$. A energia livre apresenta três pontos extremos, dois mínimos em $\Gamma$ e $\Gamma^{*}$ e um máximo em A.

Agora é possível traçar algumas conclusões a respeito do modelo de Ising a campo nulo na versão de Curie-Weiss. A altas temperaturas, como se espera, o sistema está na fase paramagnética, com uma única solução estável em $m=0$. A transição ocorre em $T=1$, isto é, quando $\tanh (m / T)$ se torna tangente à reta em $m=0$. De fato, para $m$ pequeno ${ }^{16}$, uma expansão da equação (2.22) resulta

$$
\left(\frac{1}{T}-1\right) m+\mathrm{O}\left(m^{2}\right)=0
$$

\footnotetext{
${ }^{15}$ Lembramos que o campo magnético tem unidade de energia.

${ }^{16} \mathrm{Na}$ vizinhança do ponto crítico.
} 

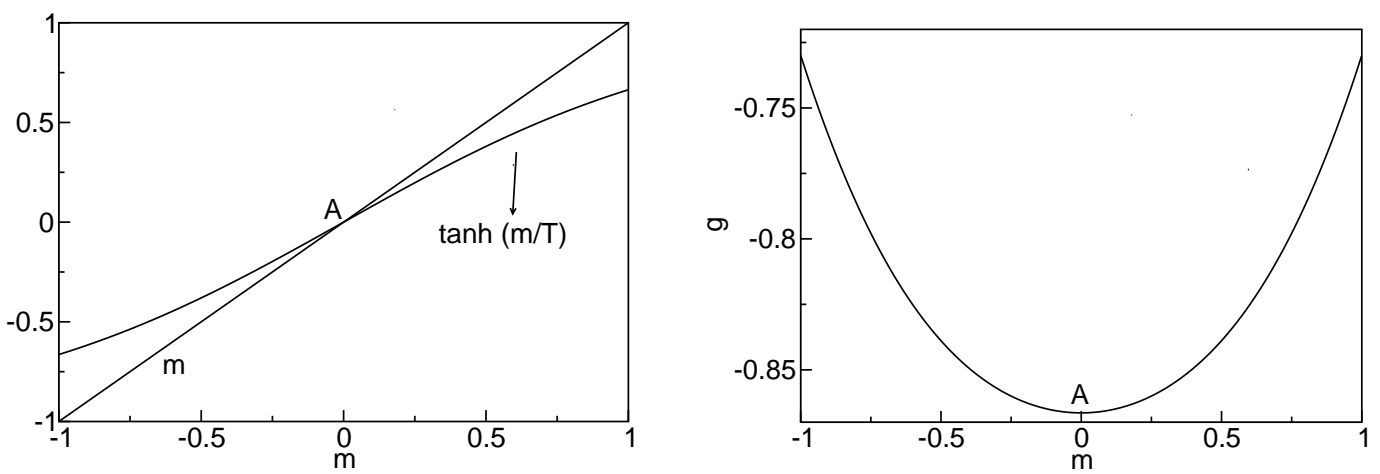

Figura 2.3: $m \times \tanh (m / T)$ e $m \times m$ (gráfico à esquerda); $m \times g$ (gráfico à direita) para $T=1.25$ e $H=0$.
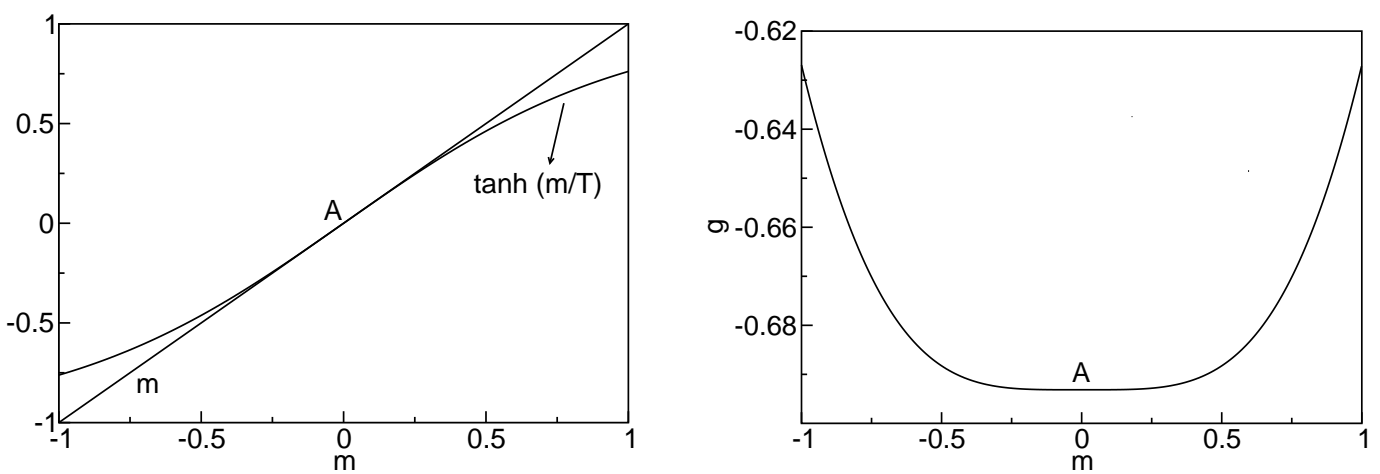

Figura 2.4: $m \times \tanh (m / T)$ e $m \times m$ (gráfico à esquerda); $m \times g$ (gráfico à direita) para $T=1$ e $H=0$.
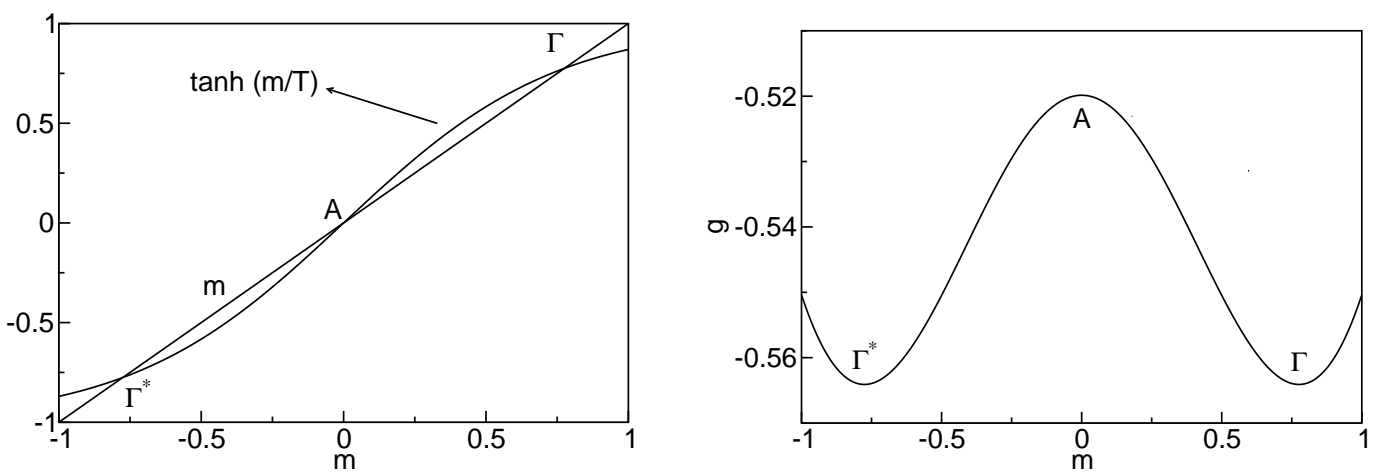

Figura 2.5: $m \times \tanh (m / T)$ e $m \times m$ (gráfico à esquerda); $m \times g$ (gráfico à direita) para $T=0.75$ e $H=0$.

de forma que, para $m \neq 0$ tão pequeno quanto se queira, devemos ter $T=T_{c}=1$. A baixas temperaturas $(T<1)$, o sistema está na fase ferromagnética, com dois mínimos 
equivalentes da energia livre nos pontos $\Gamma$ e $\Gamma^{*}$. A solução paramagnética em A, neste caso, é um máximo de $g$. É possível caracterizar a transição por meio do que chamamos parâmetro de ordem, em geral uma grandeza que é nula na fase desordenada e não nula na fase ordenada do sistema. A magnetização a campo nulo é uma boa escolha para o modelo de Ising. Uma vez que $g(\Gamma)=g\left(\Gamma^{*}\right)$, podemos optar por qualquer uma das duas soluções estáveis. No lado esquerdo da Figura 2.6, apresentamos o parâmetro de ordem do modelo de Ising, escolhendo a solução positiva $m(\Gamma)$, como função da temperatura. Note que $m(\Gamma) \rightarrow 1$ para $T \rightarrow 0$ e que $m(\Gamma) \rightarrow 0$ para $T \rightarrow 1^{-}$, e que o gráfico se assemelha aos resultados experimentais para o níquel (gráfico à direita ${ }^{17}$ ).
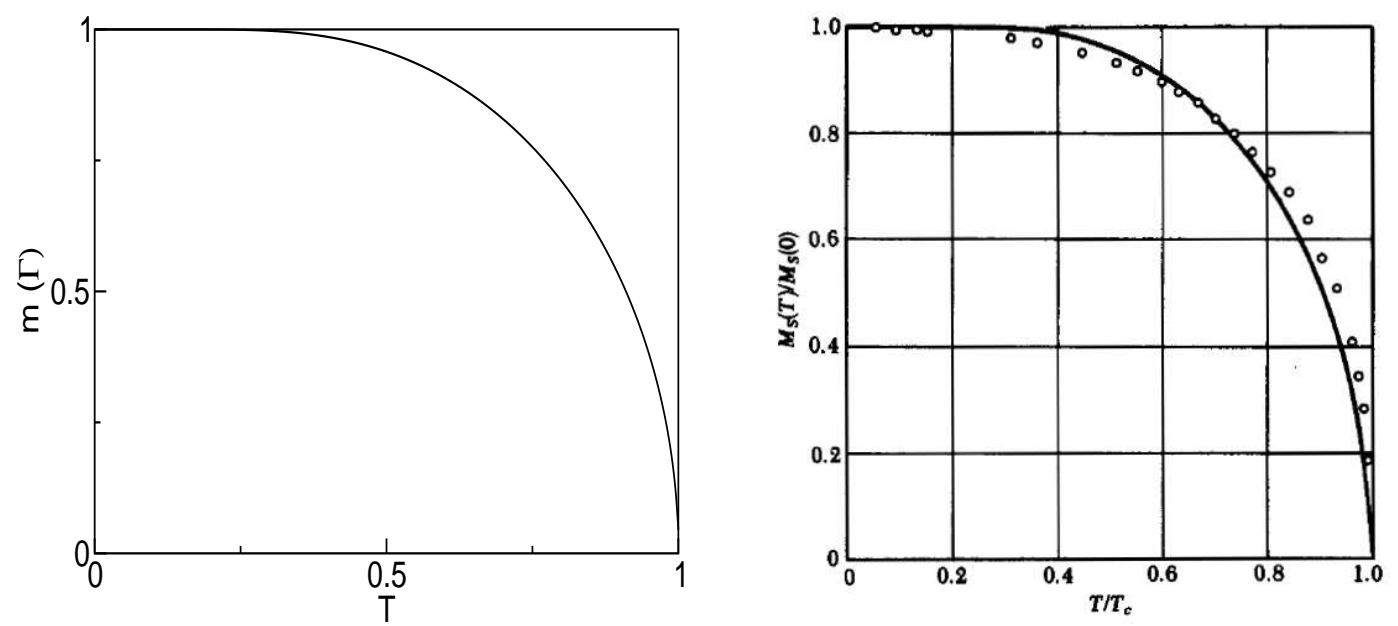

Figura 2.6: Parâmentro de ordem $m(\Gamma)$ como função da temperatura $T$ para o modelo de Ising a campo nulo na versão de Curie-Weiss (à esquerda). Os pontos do gráfico à direita são extraídos de medidas experimentais para o níquel, extraída de (Kittel, 1996).

A interação com o campo magnético destrói a transição ${ }^{18}$, como se observa na Figura (2.7). Há agora apenas uma solução em $\mathrm{B}$ com $m \neq 0$ (gráfico à esquerda) e $m$ tende a zero assintoticamente com o aumento da temperatura (gráfico à direita).

No lado esquerdo das figuras 2.8-2.10 apresentamos gráficos da magnetização versus campo magnético para $T=1.25$ (Figura 2.8), $T=1$ (Figura 2.9) e $T=0.75$ (Figura 2.10). A energia livre é apresentada no lado direito como função da magnetização. Acima

\footnotetext{
${ }^{17}$ Veja o Capítulo 15 de (Kittel, 1996).

${ }^{18} \mathrm{Na}$ realidade, podem existir até três soluções numa vizinha limitada de $H=0$, duas das quais podendo ser descartadas segundo a construção de áreas iguais de Maxwell. Umas dessas soluções é instável.
} 

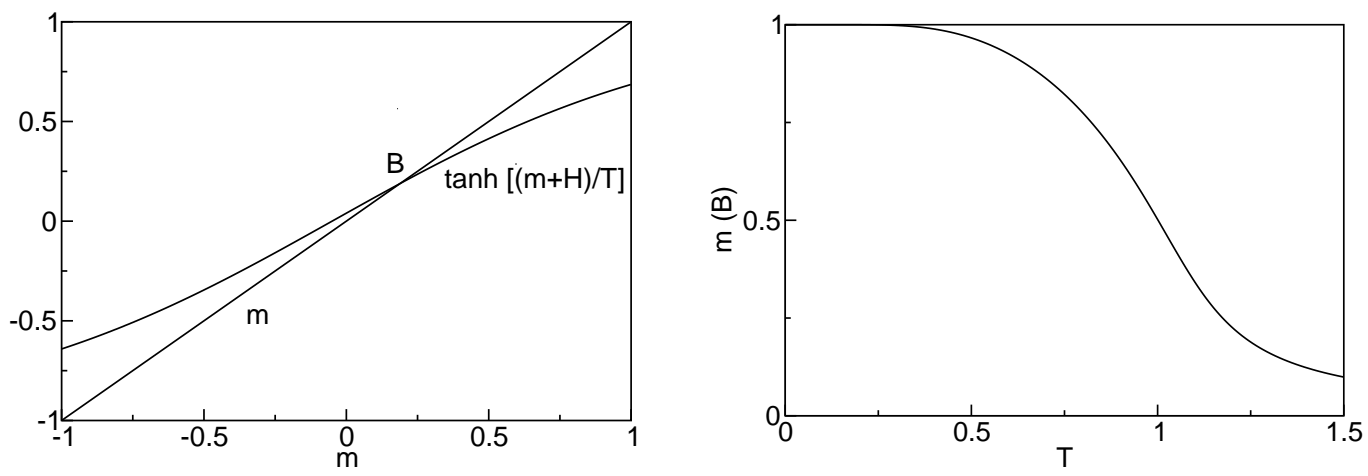

Figura 2.7: À esquerda: $m \times \tanh [(m+H) / T]$ e $m \times m$ para $H=0.05$ e $T=1.25$. À direita: magnetização como função da temperatura para $H=0.05$.

da temperatura crítica, $m$ é uma função monótona crescente de $H$, interceptando o eixo $H=0$ em $m=0$ (Ponto A). A energia livre $g$, que agora é de fato um potencial termodinâmico ${ }^{19}$, é uma função côncava de $m$ com um máximo em $\mathrm{A}$. Em $T=T_{c}=1$, $m$ intercepta o eixo $H=0$ tangencialmente e $g$ apresenta um achatamento em torno do ponto A.
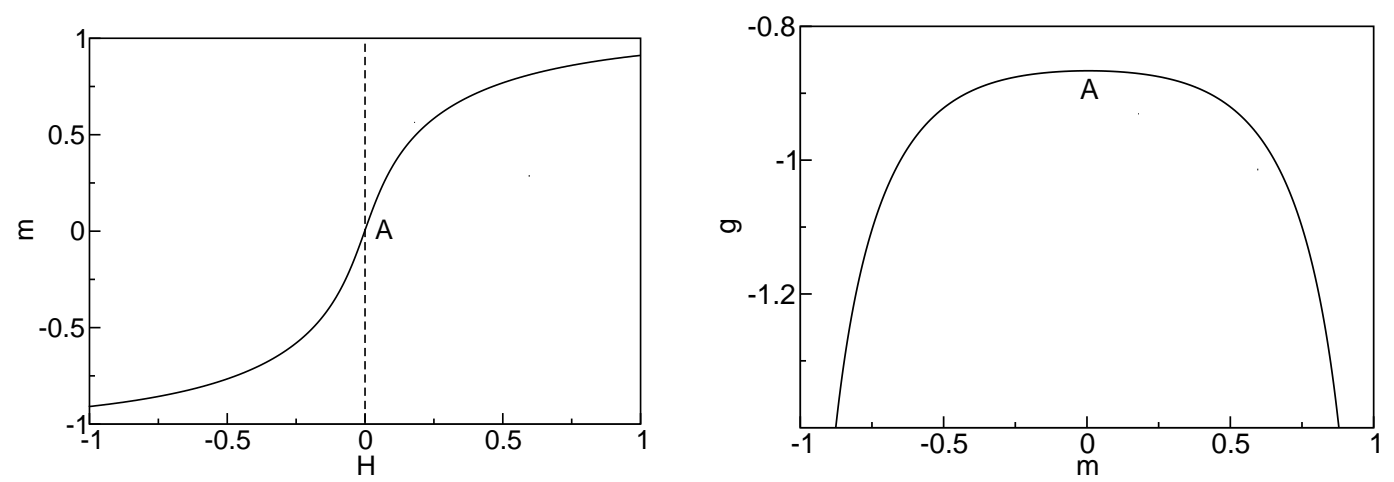

Figura 2.8: Magnetização versus campo magnético (gráfico à esquerda) e energia livre versus magnetização (gráfico à direita) para $T=1.25$.

Para $T<T_{c}$ a análise é um pouco mais delicada. Para $T=0.75$ pode haver até três valores de $m$ para o mesmo campo. Os pontos A, $\Gamma$ e $\Gamma^{*}$ são análogos aos da Figura 2.5. Note que, abaixo da linha tracejada no gráfico $m \times g$ da Figura 2.10, sempre é possível

\footnotetext{
${ }^{19}$ Nas curvas apresentadas nas figuras 2.3-2.5, os valores de $g$, exceto no ponto mínimo, eram imateriais. Deste ponto de vista $g$ deveria ser visto como um funcional de $m$, dado que $m$ deveria satisfazer à equação (2.22). Além disso $g=g(H, T)$, de forma que os gráficos $m \times g$ nas figuras 2.8-2.10 correspondem, de fato, à função implícita $g(H(m), T)$.
} 

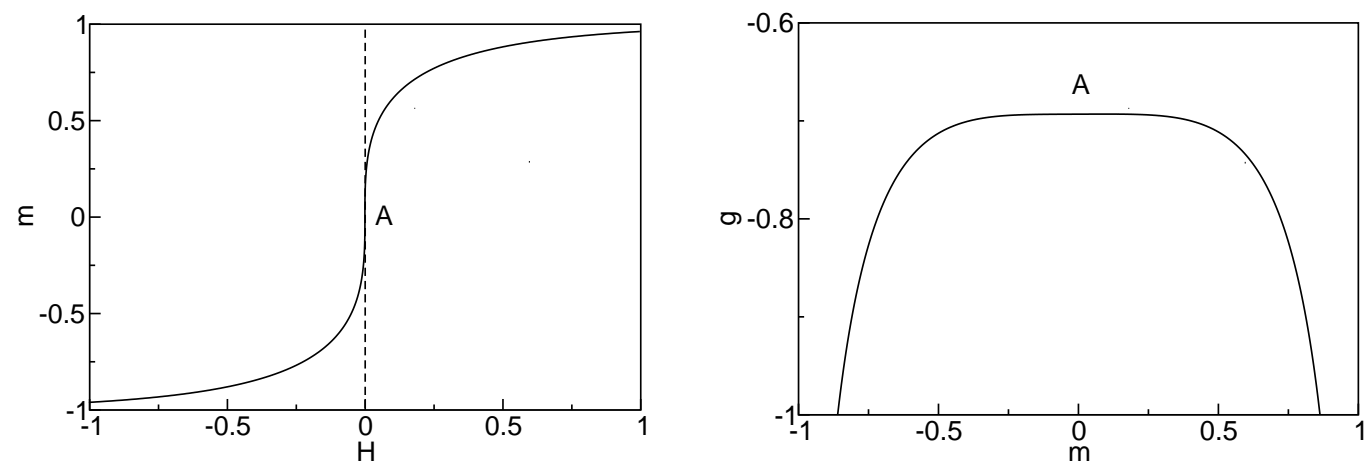

Figura 2.9: Magnetização versus campo magnético (gráfico à esquerda) e energia livre versus magnetização (gráfico à direita) para $T=1$.

escolher dois pontos $\left(m_{1}, g\left(m_{1}\right)\right)$ e $\left(m_{2}, g\left(m_{2}\right)\right)$, com $m_{2}>m_{1}$, tal que a reta que os intercepta esteja acima de $g(m)$ para $m \in\left(m_{1}, m_{2}\right)$, ou seja, a concavidade de $g(m)$ é violada. Entretanto, $g$ deve ser uma função côncava de $m$ por uma questão de estabilidade termodinâmica $^{20}$. Uma forma de contornar esse problema é substituir $g(m)$ pela menor função côncava maior ou igual a $g$, de forma análoga à construção do chamado envelope convexo $^{21}$. No nosso exemplo basta descartarmos $g(m)$ abaixo do ramo tracejado no lado direito da Figura 2.10 e traçar uma reta entre $\Gamma^{*}$ e $\Gamma$. Este procedimento é equivalente à construção de áreas iguais de Maxwell, cuja ideia consiste em traçar uma reta vertical na região de instabilidade da curva $m \times H$ de maneira que a área entre a reta e a curva no intervalo $\left(m_{a}, m_{b}\right)$ seja igual à área entre a reta e a curva no intervalo $\left(m_{b}, m_{c}\right)$, onde $m_{a}<m_{b}<m_{c}$ são os três pontos de interseção entre a reta vertical e a curva $m \times H$. Essa reta é simplesmente $H=0$ no nosso caso. A curva corrigida deve, portanto, ter um salto de $m\left(\Gamma^{*}\right)<0$ a $m(\Gamma)>0$ em $H=0$ (gráfico interno à esquerda da Figura 2.10), o que caracteriza uma transição de primeira ordem. O caráter instável da solução

${ }^{20}$ Estabilidade termodinâmica requer que, a temperatura fixa,

$$
\frac{\partial^{2} g}{\partial H^{2}}<0
$$

o que implica

$$
\frac{\partial^{2} g}{\partial m^{2}}\left(\frac{\partial m}{\partial H}\right)^{2}+\frac{\partial g}{\partial m} \frac{\partial^{2} m}{\partial m \partial H} \frac{\partial m}{\partial H}<0
$$

de forma que $\partial^{2} g / \partial m^{2}<0$.

${ }^{21} \mathrm{O}$ desenvolvimento padrão consiste em restaurar a convexidade de $H m+g(m)$, o que permite tomar o supremo em $H$ de forma unívoca, caracterizando uma espécie de transformada de Legendre bem definida. A ideia porém é a mesma. 
decrescente de $m$ também pode ser visto por meio de um gráfico de $g$ como função de $H$, inserido no gráfico da energia livre. Embora duas das soluções sejam sempre estáveis para um intervalo de valores de $H$, a solução correta deve ser aquela de menor energia livre. A terceira solução, além de possuir maior energia livre, é também instável (convexa) do ponto de vista termodinâmico.
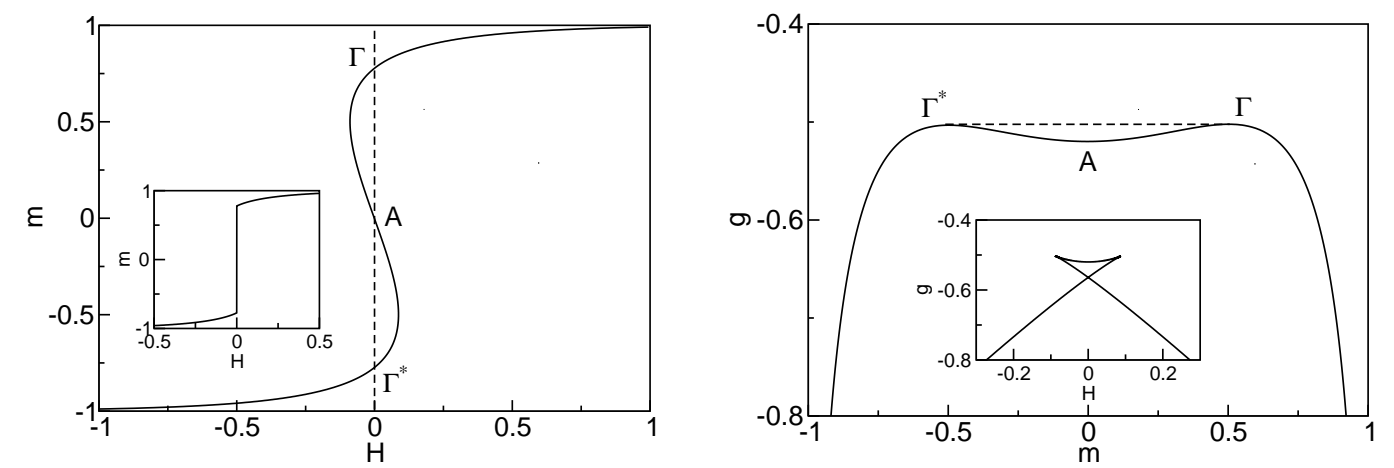

Figura 2.10: Magnetização versus campo magnético (gráfico à esquerda), magnetização corrigida versus campo magnético (gráfico interno à esquerda), energia livre versus magnetização (gráfico à direita) e energia livre versus campo magnético (gráfico interno à direita), para $T=0.75$.

Podemos agora discutir o diagrama de fases campo versus temperatura do modelo de Curie-Weiss (Figura 2.11). Para $T<T_{c}$, existe ordenamento ferromagnético com magnetização positiva para $H>0$ e magnetização negativa para $H<0$. A linha $H=0$ no intervalo de temperaturas $(0,1)$ caracteriza uma transição de primeira ordem, e termina no ponto crítico $\mathrm{C}$. Acima de $T_{c}$ o sistema está na fase paramagnética, $m$ varia continuamente de valores negativos a positivos (ou vice e versa). Além disso, a susceptibilidade magnética a campo nulo $\chi$, a $T$ constante, obtida da equação,

$$
\chi=\left.\frac{\partial m}{\partial H}\right|_{H=0}=\frac{T\left(1-m^{2}\right)}{T-\left(1-m^{2}\right)},
$$

obedece à famosa lei de Curie -Weiss para $T>1$, onde $m=0$, e diverge no ponto crítico (Figura 2.12). 


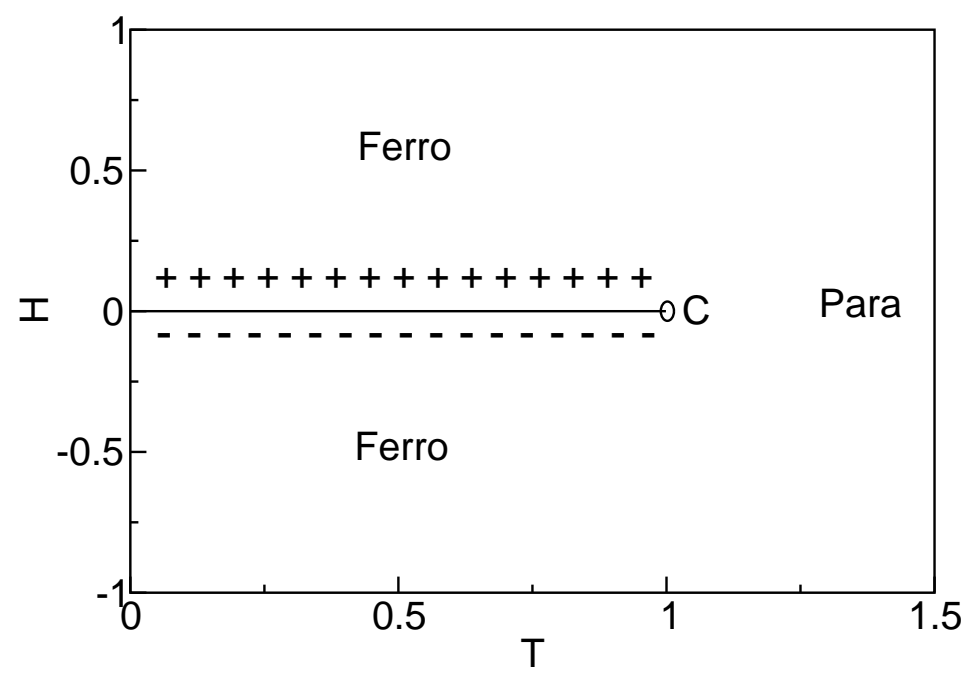

Figura 2.11: Diagrama de fases campo versus temperatura do modelo de Ising. Os termos Ferro e Para indicam ordenamento ferromagnético e paramagnético respectivamente. A linha $H=0$ no intervalo $(0,1)$ caracteriza uma transição de primeira ordem entre as fases ferromagnéticas positiva e negativa. O ponto C é o ponto crítico do sistema.

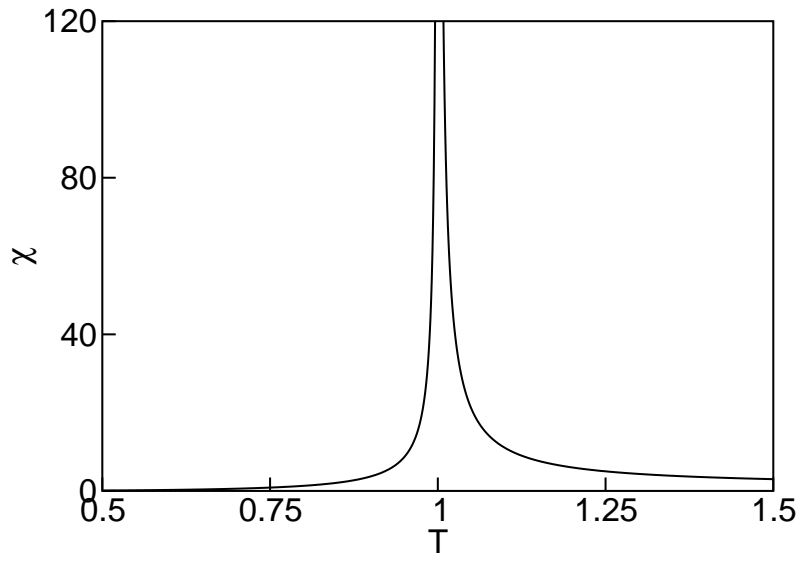

Figura 2.12: Susceptibilidade magnética a campo nulo como função da temperatura para o modelo de Curie-Weiss.

\subsection{Teoria de Landau, expoentes críticos e o critério de Ginz- burg}

$\mathrm{Na}$ vizinhança do ponto crítico, a campo magnético nulo, uma expansão da energia livre (2.23) resulta

$$
g=-T \ln 2+\frac{1}{2}\left(1-\frac{1}{T}\right)_{22} m^{2}+\frac{1}{12} \frac{m^{4}}{T^{3}}+\mathrm{O}\left(m^{6}\right) .
$$


A condição de ponto estacionário tem então a forma

$$
\left(1-\frac{1}{T}\right) m+\frac{1}{3} \frac{m^{3}}{T^{3}}+\mathrm{O}\left(m^{5}\right)=0
$$

Assim, o ponto crítico pode ser obtido considerando $m$ não nulo, tão pequeno quanto se queira, que ocorre para (2.27) em $T=1$. Segue ainda da (2.27), para $T<1$,

$$
m^{2}=3 T^{3}\left(\frac{1}{T}-1\right)+\mathrm{O}\left(m^{4}\right)
$$

A segunda derivada de $g$ em $m$ resulta então

$$
\frac{\partial^{2} g}{\partial m^{2}}=\frac{2}{T}(1-T)+\mathrm{O}\left(m^{4}\right)>0
$$

indicando que a solução $m \neq 0$, para $T<1$ na vizinhança do ponto crítico, é estável. Para isso admitimos que o coeficiente do termo de ordem quatro é positivo ${ }^{22}$.

Vale notar que apenas termos de ordem par aparecem na expansão (2.26), e que o termo de segunda ordem é da forma $A\left(T-T_{c}\right)$. De fato, partindo de resultados obtidos de um modelo microscópico, o que fizemos foi estabelecer contato com a teoria fenomenológica de Landau para transições de fases ${ }^{23}$ (Landau, Lifshitz, e Pitaevskii, 1980). A proposta de Landau se baseia numa expansão geral, em torno do ponto crítico, da energia livre em termos de um parâmetro de ordem adequado. As simetrias do parâmetro de ordem determinam os vínculos a serem obedecidos pelos coeficientes dessa expansão. Para o modelo de Ising, por exemplo, a simetria de inversão de spins e campos implica, em última análise, que deve haver apenas termos de ordem $n$ par em $m^{n}$, e que acoplamentos com o campo magnético devem ser potências de $H m$. Nesse caso, podemos escrever (Cardy, 1996)

$$
g_{\mathrm{MF}}(H, T ; m)=a+b t m^{2}+c m^{4}+d H m+\mathrm{O}\left(m^{6}\right)
$$

onde $a, b, c$ e $d$ dependem apenas fracamente da temperatura reduzida $t \equiv\left(T-T_{c}\right) / T_{c}$. Podemos aplicar a mesma análise que utilizamos nas equações (2.26-2.29). Além disso, podemos obter a dependência de várias grandezas para $t \rightarrow 0^{-}$, ou seja calcular os diversos

\footnotetext{
${ }^{22}$ Como se pode verificar.

${ }^{23}$ Essa teoria se assemelha à teoria da elasticidade, onde a estrutura atômica microscópica é ignorada e o sólido tratado como um contínuo (Ashcroft e Mermin, 1976).
} 
expoentes críticos. Segue da equação (2.28) que

$$
m \sim(-t)^{\beta}, \quad \text { para } t \rightarrow 0^{-},
$$

com

$$
\beta=1 / 2 \text {. }
$$

Outros expoentes associados à divergência da susceptibilidade $\chi(H, T)$,

$$
\chi(0, T) \sim(-t)^{-\gamma}, \quad \text { para } t \rightarrow 0^{-},
$$

e à dependência da magnetização no campo magnético em $t=0$,

$$
m\left(H, T_{c}\right) \sim H^{1 / \delta}, \quad \text { para } H \rightarrow 0
$$

tem os valores clássicos

$$
\gamma=1 \text { e } \quad \delta=3
$$

O comportamento do calor específico a campo nulo na vizinhança do ponto crítico pode ser descrito por

$$
c(0, T) \sim(-t)^{-\alpha}, \quad \text { para } t \rightarrow 0^{-},
$$

Segundo um formalismo de campo médio, o calor específico apresenta uma descontinuidade na temperatura crítica, permanecendo finito antes e depois da transição. Por se aproximar de $t=0$ de forma perfeitamente analítica, associa-se o expoente crítico $\alpha=0$, resultado que não persiste para $d<4$. Esses expoentes também são conhecidos como expoentes de Landau.

A teoria de campo médio funciona muito bem na descrição qualitativa do sistema longe da criticalidade. Como se sabe, os expoentes críticos das equações (2.32) e (2.35) diferem dos obtidos por meio de soluções exatas, simulações, e de experimentos. Correções para esses resultados devem levar em conta o termo em $\delta \sigma \delta \sigma^{\prime}$ que desprezamos em (2.7). De fato, a aproximação de campo médio se torna auto-consistente apenas quando o valor esperado de

$$
\frac{J}{N} \sum_{(i, j)} \delta \sigma_{i} \delta \sigma_{j}
$$


calculado usando no fator de Boltzmann a mesma energia aproximada presente em (2.7), for muito menor que a densidade de energia. Essa análise, para temperaturas próximas à temperatura crítica, onde em geral o comprimento de correlação diverge, resulta na relação (Cardy, 1996)

$$
\xi^{4-d} \ll R^{4}
$$

onde

$$
R=\sum_{r} r^{2} J(r) / \sum_{r} J(r)
$$

dá uma ideia do alcance da interação de troca ${ }^{24}$. Esse resultado é conhecido como critério de Ginzburg, a menos de alguns fatores de $2 \pi$. O aumento do comprimento de correlação na vizinhança do ponto crítico leva a uma violação da relação (2.38) para $d<4$, o que explica a discrepância dos resultados de campo médio nessa região. Entretanto, ao mesmo tempo que longe do ponto crítico a relação (2.38) é muitas vezes satisfeita, fornecendo assim um panorama qualitativo adequado. Em alguns sistemas, como supercondutores do tipo I, o valor de $R$ é grande, da ordem do tamanho de um par de Cooper, de forma que os resultados de campo médio são bons mesmo para regiões muito próximas ao ponto crítico. Para $d>4$ os termos de correção são bem menos singulares e apenas modificam as amplitudes sem alterar os expoente críticos do sistema. A dimensão $d_{\text {sup }}=4$ é chamada dimensão crítica superior do modelo de Ising de curto alcance.

\subsection{Modelo de Ising com campos aleatórios}

Considere agora uma interação com um campo magnético aleatório e não uniforme, satisfazendo uma dada distribuição de probabilidades. Esta pode ser uma distribuição gaussiana, ou uma distribuição bimodal, por exemplo ${ }^{25}$. A princípio vamos considerar a

\footnotetext{
${ }^{24}$ Note que para escrever $R$ de acordo com a equação (2.39) tivemos que abandonar a notação discreta baseada nos sítios da rede $i$ e $j$. Uma maneira de fazer isso consiste em escrever a hamiltoniana de Ising na forma

$$
\mathcal{H}=-\sum_{r, r^{\prime}} J\left(r-r^{\prime}\right) \sigma(r) \sigma\left(r^{\prime}\right)
$$

que é muito mais adequada a uma análise de Fourier. O vínculo de interação entre vizinhos mais próximos está incorporado a $J(r)$ agora.

${ }^{25} \mathrm{~A}$ escolha da distribuição pode ser bastante relevante. No modelo de Curie-Weiss diluído, por exemplo, a existência de um ponto tricrítico depende dessa escolha.
} 
hamiltoniana mais geral, na versão de Curie-Weiss,

$$
\mathcal{H}=-\frac{J}{2 N}\left(\sum_{i=1}^{N} \sigma_{i}\right)^{2}-\sum_{i=1}^{N} h_{i} \sigma_{i}
$$

onde $h_{i}$ são variáveis aleatórias temperadas ${ }^{26}$, ou seja, fixas, independentes e identicamente distribuídas com probabilidade $P\left(h_{i}\right)$. Um modelo desse tipo é conhecido como modelo de Ising com campos aleatórios ${ }^{27}$. Não vamos explorar a equivalência com um modelo diluído antiferromagnético com um campo uniforme, demonstrada por Fishman, Aharony e Cardy ${ }^{28}$. Nosso interesse repousará antes sobre dois aspectos: 1) a introdução de alguns métodos de análise de sistemas desordenados; 2) a conexão, como veremos no Capítulo 5, com tensões anisotrópicas dos chamados elastômeros nemáticos.

Para uma configuração fixa de campos aleatórios ${ }^{29}$, o termo quadrático pode ser linearizado por meio de uma identidade gaussiana, de forma semelhante à equação (2.14), que resulta

$$
Z=\sqrt{\frac{\beta J N}{2 \pi}} \int_{-\infty}^{+\infty} d y \exp \left(-\frac{1}{2} N \beta J y^{2}\right) \operatorname{Tr} \exp \left[\beta \sum_{i}\left(J y+h_{i}\right) \sigma_{i}\right] .
$$

A soma implícita no traço agora se fatoriza, de forma que

$$
\begin{aligned}
Z & =\sqrt{\frac{\beta J N}{2 \pi}} \int_{-\infty}^{+\infty} d y \exp \left(-\frac{1}{2} N \beta J y^{2}\right) \prod_{i}\left[2 \cosh \beta\left(J y+h_{i}\right)\right] \\
& =\sqrt{\frac{\beta J N}{2 \pi}} \int_{-\infty}^{+\infty} d y \exp \left(-\frac{1}{2} N \beta J y^{2}\right) \exp \left[\sum_{i} \ln 2 \cosh \beta\left(J y+h_{i}\right)\right]
\end{aligned}
$$

Uma vez que $h_{i}$ é uma variável aleatória com distribuição de probabilidades $P\left(h_{i}\right)$, $\ln 2 \cosh \beta\left(J y+h_{i}\right)$ também é uma variável aleatória satisfazendo a mesma distribuição, de forma que, de acordo com a lei dos grandes números

$$
\lim _{N \rightarrow \infty} \frac{1}{N} \sum_{i=1}^{N} \ln 2 \cosh \beta\left(J y+h_{i}\right)=\langle\ln 2 \cosh \beta(J y+h)\rangle_{h},
$$

onde $\langle\cdots\rangle_{h}$ é o valor esperado definido pela distribuição de probabilidades $P(h)$. Podemos então escrever, descartando termos de ordem menor que $N$,

$$
Z=\sqrt{\frac{\beta J N}{2 \pi}} \int_{-\infty}^{+\infty} d y e^{-N \psi}
$$

\footnotetext{
${ }^{26}$ Quenched. Veja o Capítulo 1 para uma introdução aos tipos de desordem.

${ }^{27}$ Random-field Ising model.

${ }^{28}$ Para referências, veja o Capítulo 2 de (Dominicis e Giardina, 2006).

${ }^{29}$ Veja comentário ao final deste capítulo.
} 
onde

$$
\psi=\psi(y)=\frac{1}{2} \beta J y^{2}-\langle\ln 2 \cosh \beta(J y+h)\rangle_{h} .
$$

Novamente, o método assintótico de Laplace resulta

$$
Z \approx e^{-N \psi(y *)}
$$

com $y^{*}$ dado por

$$
\left.\frac{\partial \psi}{\partial y}\right|_{y=y^{*}}=0,
$$

ou seja, $y^{*}$ deve satisfazer a equação auto-consistente

$$
y^{*}=\left\langle\tanh \beta\left(J y^{*}+h\right)\right\rangle_{h},
$$

que é semelhante à (2.19). A energia livre é dada por

$$
g=\frac{1}{2} J y^{* 2}-k T\left\langle\ln 2 \cosh \beta\left(J y^{*}+h\right)\right\rangle_{h}
$$

Uma vez que $y^{*}$ corresponde de fato à magnetização do sistema ${ }^{30}$, podemos escrever novamente as energias em unidades de $J$ e a temperatura em unidade de $k / J$, tal que

$$
g=\frac{1}{2} m^{2}-T\left\langle\ln 2 \cosh \left(\frac{m+h}{T}\right)\right\rangle_{h},
$$

onde

$$
m=\left\langle\tanh \left(\frac{m+h}{T}\right)\right\rangle_{h} .
$$

Uma vez definida a distribuição de campos aleatórios, podemos proceder com uma análise semelhante à que fizemos para o modelo de Curie-Weiss para obter os pontos críticos, expoentes críticos, etc.

Para resolver o problema de campos aleatórios, supostos fixos, ou temperados, calculamos a função de partição para um conjunto fixo $\left\{h_{i}\right\}$, e utilizamos a lei dos grandes números em (2.44) para extrair o conhecimento da distribuição de probabilidades $P\left(h_{i}\right)$. Nem sempre é possível adotar um procedimento tão direto. De fato, grandezas que podem ser calculadas dessa maneira constituem uma subclasse de grandezas que satisfazem uma

\footnotetext{
${ }^{30}$ Para verificar essa asserção basta considerar uma hamiltoniana com um termo de interação com um campo uniforme $H$, tomar a derivada em $H$ ao final e o limite de $H \rightarrow 0$.
} 
propriedade mais geral, conhecida como auto-mediância ${ }^{31}$. Grandezas auto-mediantes são aquelas que possuem o mesmo valor para quase todas ${ }^{32}$ as configurações de variáveis aleatórias, por conseguinte igual também ao valor médio,

$$
\phi\left(\left\{h_{i}\right\}\right)=\left\langle\phi\left(\left\{h_{i}\right\}\right)\right\rangle_{\left\{h_{i}\right\}},
$$

onde $\phi\left(\left\{h_{i}\right\}\right)$ denota o valor da grandeza para um dado conjunto $\left\{h_{i}\right\}$, e a média no lado direito é tomada dobre os valores de $h_{i}$. Em geral, argumenta-se que grandezas extensivas, como a energia livre, sejam auto-mediantes (Brout, 1959). O chamado método das réplicas provê uma abordagem sistemática na obtenção das propriedades termodinâmicas de sistemas com desordem temperada. Voltaremos a esse tópico, com mais detalhes, no próximo capítulo.

\footnotetext{
${ }^{31}$ Self-averaging.

${ }^{32}$ Todas a menos de um conjunto de medida nula.
} 


\section{Capítulo 3}

\section{Efeitos de compressibilidade no modelo de vidros de spins de}

\section{Sherrington-Kirkpatrick}

Vidros de spins são representantes arquetípicos de sistemas com desordem temperada. Grosso modo, esses materiais são conjuntos de momentos magnéticos que apresentam uma fase congelada e desordenada a baixas temperaturas. Tal definição, que buscaremos aperfeiçoar adiante, foi utilizada para rotular uma classe de ligas metálicas estudada exaustivamente a partir da década 70, cujos representantes típicos são soluções diluídas de impurezas magnéticas em hospedeiros metálicos nobres, como CuMn e AuFe. No CuMn, por exemplo, os íons magnéticos de manganês, inseridos aleatoriamente no volume da amostra, magnetizam, em suas vizinhanças, os elétrons de condução do cobre metálico. As magnetizações induzidas, positivas ou negativas dependendo da distância, produzem campos que atuam sobre as demais impurezas magnéticas. O resultado é um sistema de spins com interações competitivas e aleatórias (Fischer e Hertz, 1991). São essas interações que dão origem à chamada fase vidro de spins, cujo nome foi dado por Brian Cooles no final da década de 60 devido às semelhanças com vidros estruturais convencionais, que também apresentam uma fase congelada "amorfa", assim como uma dependência linear do calor específico a baixas temperaturas (Sherrington, 2008).

Neste capítulo introduzimos um modelo de vidros de spins de Sherrington-Kirkpatrick 
com graus de liberdade elásticos. No ensemble das pressões, aplicamos o método das réplicas para analisar o comportamento crítico e o diagrama de fases do modelo, estabelecendo contato com os diagramas obtidos experimentalmente para misturas de cristais ferro e antiferroelétricos com ligações de hidrogênio (Liarte et al., 2009). Na seção 3.1 fazemos uma breve revisão histórica e conceitual sobre a teoria de vidros de spins. Na seção 3.2 apresentamos as motivações do estudo de vidros de spin com graus de liberdade elásticos. O modelo é definido na seção 3.3. Utilizamos o método das réplicas na seção 3.4, e analisamos a solução com simetria de réplicas na seção 3.5. Por fim, discutimos o diagrama de fases e conexões com experimentos na seção 3.6.

\subsection{O sistema: Vidros de spins}

Para descrever vidros de spins, usamos a palavra congelada, indicando momentos magnéticos locais não nulos $\left(\left\langle S_{i}\right\rangle \neq 0\right.$, onde $\langle\cdots\rangle$ denota uma média térmica). O termo desordem entra no fato de não existir ordenamento ferromagnético $\left(N^{-1} \sum_{i}\left\langle S_{i}\right\rangle=0\right)$, ou antiferromagnético $\left(N^{-1} \sum_{i} e^{-i \boldsymbol{k} \cdot \boldsymbol{r}_{i}}\left\langle S_{i}\right\rangle=0\right)$ de longo alcance. Experimentalmente, ao resfriar a amostra a uma temperatura suficientemente baixa, o congelamento dos spins leva a uma brusca diminuição da susceptibilidade magnética de forma similar ao que acontece com antiferromagnetos ${ }^{1}$ (Figura 3.1). Esse comportamento foi explorado exaustivamente e habilmente por experimentais desde o início da década de 70 (Cannella e Mydosh, 1972; Fischer e Hertz, 1991). Do ponto de vista teórico, foi o trabalho de Edwards e Anderson que fomentou a investigação analítica desses materiais (Edwards e Anderson, 1975). Há uma citação de David Sherrington que expressa bem o papel do trabalho de Edwards e Anderson no desenvolvimento posterior da teoria de vidros de spins: "But still theoretical work was minimal until Edwards and Anderson (1975) produced a paper that at one fell swoop recognised the importance of the combination of frustration and quenched disorder as fundamental ingredients, introduced a more convenient model, a new and novel method of analysis, new types of order parameters, a new mean field theory, new approximation

\footnotetext{
${ }^{1}$ Experimentos de espalhamento de nêutrons para vidros de spin não exibem os picos de Bragg indicando ordenamento de longo alcance onde seria esperado para antiferromagnetos.
} 
techniques and the prediction of a new type of phase transition apparently explaining the observed susceptibility cusp. This paper was a watershed." (Sherrington, 2008).

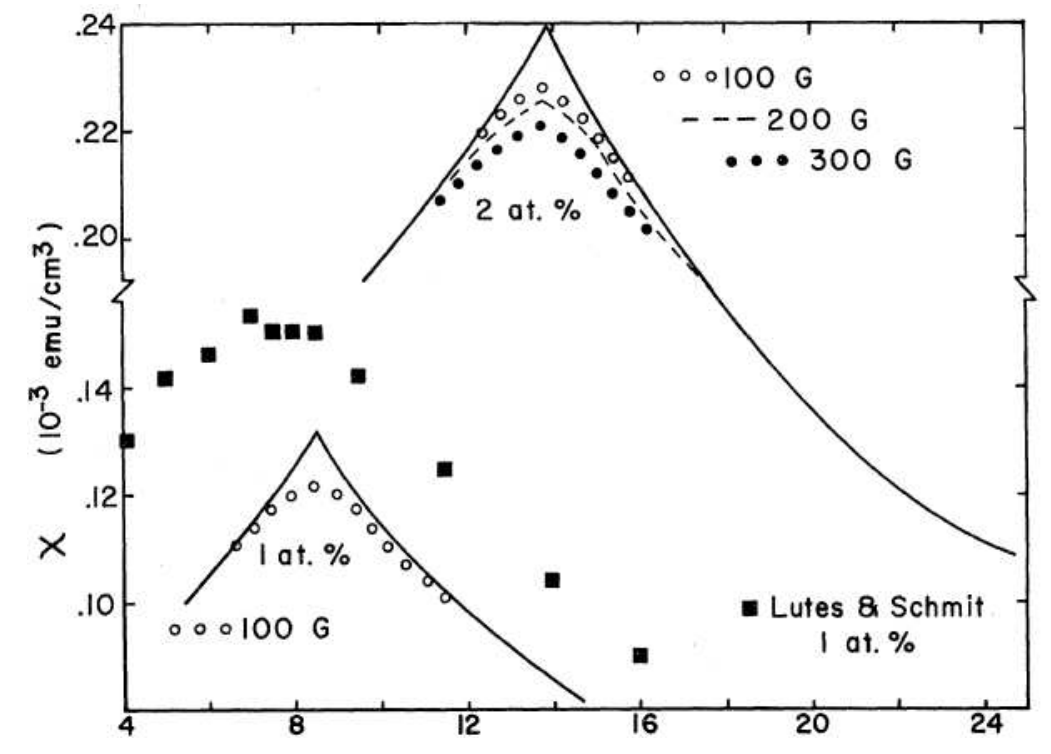

Figura 3.1: Susceptibilidade magnética como uma função da temperatura, a campo nulo e não-nulo, para o AuFe. Extraído de (Cannella e Mydosh, 1972).

A linha iniciada por Edwards e Anderson, seguida por Sherrington e Kirkpatrick, Parisi, culminando com Guerra e Toninelli, e finalmente Talagrand ${ }^{2}$, não é a única abordagem para vidros de spins. Um outro modelo popular foi proposto por Daniel Fisher e David Huse em meados da década de 80 e é conhecido como droplet model ${ }^{3}$ (Fisher, Grinstein, e Khurana, 1988). Os diferentes cenários que essas abordagens produzem tem gerado debates estimulantes, não resolvidos a meu ver, sobre a natureza da fase vidro de spins em dimensão finita. Apesar dessa incerteza, é certo que a teoria de campo médio criada em torno do trabalho de Edwards e Anderson estabeleceu pilares para as mais diversas aplicações, o que tornou os vidros de spins um verdadeiro paradigma de sistemas com desordem temperada, ao ponto de serem comparados a osciladores harmônicos ${ }^{4}$. Problemas

\footnotetext{
${ }^{2}$ Minha classificação pessoal deixou de fora um sem-número de trabalhos relacionados de importância capital, entre eles, por exemplo, a análise de estabilidade da solução com simetria de réplicas, devida a Jairo de Almeida e David Thouless (Almeida e Thouless, 1978).

3 "Modelo de gotas", numa tradução livre.

${ }^{4} \mathrm{~A}$ comparação, decorrente da vasta aplicabilidade da teoria de vidros de spins, é atribuída a Ton Coolen (Barra, Guerra, e Mingione, 2011)
} 
em biologia, economia e processamento de informação são alguns exemplos onde a teoria de vidros de spin tem sido aplicada (Mezard, Parisi, e Virasoro, 1987; Nishimori, 2001).
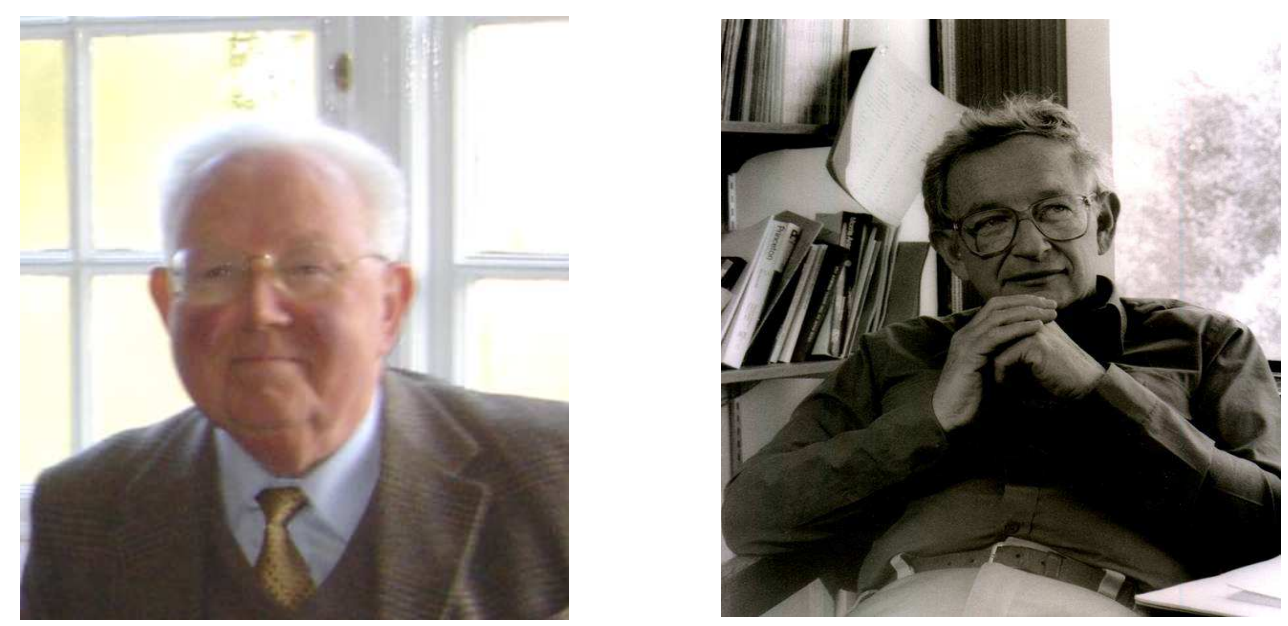

Figura 3.2: Sir Samuel Edwards (foto à esquerda) e Philip Anderson (foto à direita). Fonte: www.wikipedia.com (maio de 2011)

Para entender melhor o conceito de frustração, considere relações de amizades entre três pessoas, Alice (A), Bob (B) e Charles (C) (Figura 3.3). Alice é amiga de Bob (+) e de Charles (+), mas Bob e Charles são inimigos (-). Suponha que haja um tipo de eleição com duas chapas, $(\uparrow)$ e $(\downarrow)$, como a eleição atual para a diretoria da SBF, por exemplo, e que apenas as relações de amizade sejam relevantes na decisão de cada votante ${ }^{5}$. Tendo Charles feito sua opção pela chapa $(\uparrow)$, Bob imediatamente escolhe a chapa $(\downarrow)$, o que deixa Alice em sérios apuros, uma vez que qualquer opção a levará a romper um de seus vínculos de amizade, uma situação frustrante. Vamos agora escrever o problema de Alice em linguagem matemática. Para isso, associamos a cada um dos três elementos (A, B e C) uma variável $S$, como a componente $z$ do spin de um sistema magnético, que pode ter o valor $+1(\uparrow)$ ou $-1(\downarrow)$. A hipótese de que apenas relações de amizade sejam relevantes significa que o sistema tende a "minimizar" uma certa energia $E$ que pode ser modelada por

$$
E=-\left(E_{A B}+E_{A C}+E_{B C}\right),
$$

${ }^{5} \mathrm{O}$ que, obviamente, não é o nosso caso. 
onde

$$
E_{I J}=J_{I J} S_{I} S_{J}
$$

O parâmetro $J_{I J}$ indica interação ferromagnética quando positivo (ou uma relação de amizade no exemplo anterior), e antiferromagnética quando negativo. O problema reside no fato de todas as quatro configurações que satisfazem a condição $J_{B C}$ negativo terem o mesmo valor de energia $E=-1$. De forma geral, para $\left(J_{A B}, J_{A C}, J_{B C}\right)=(+,+,-)$ fixo ${ }^{6}$, há seis estados com energia -1 ,

$$
\{(\uparrow, \uparrow, \downarrow),(\downarrow, \uparrow, \downarrow),(\uparrow, \downarrow, \uparrow),(\downarrow, \downarrow, \uparrow),(\uparrow, \uparrow, \uparrow),(\downarrow, \downarrow, \downarrow)\}
$$

e dois estados com energia +3 ,

$$
\{(\downarrow, \uparrow, \uparrow),(\uparrow, \downarrow, \downarrow)\} \text {. }
$$

Frustração está associada ao fato de não haver estados onde as variáveis microscópicas satisfazem todos os "vínculos" impostos pelas interações, o que produz degenerescência no estado fundamental, que aumenta com o tamanho do sistema. Para os modelos de vidros de spins que abordaremos esse conceito pode ser confundido com competição entre ligações ferro e antiferromagnéticas. A ideia porém é mais geral. Considere o caso em que $\left(J_{A B}, J_{A C}, J_{B C}\right)=(-,-,-)$. Esse sistema compõe o modelo de Ising antiferromagnético na rede triangular, e é igualmente frustrado, embora não competitivo. A frustração nesse caso é uma propriedade topológica da rede.

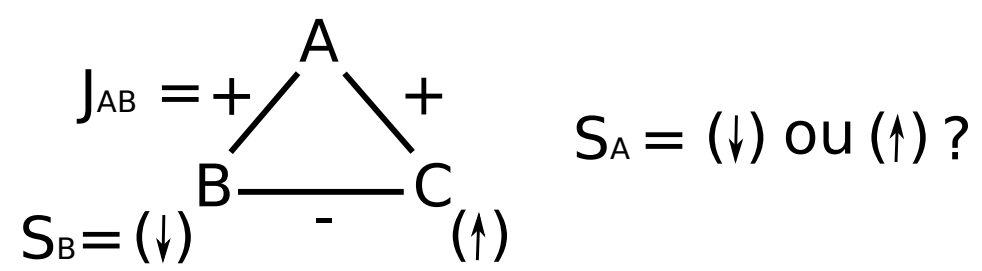

Figura 3.3: Conjunto de variáveis (A, B e C) com interações frustradas.

O termo desordem em modelos de vidros de spins indica que as interações devem ser, ao menos parcialmente, aleatórias ${ }^{7}$. O tratamento estatístico de variáveis de desordem

\footnotetext{
${ }^{6}$ Para vidros de spins e outros sistemas com desordem, a aproximação de $\boldsymbol{J}$ fixo é razoável.

${ }^{7}$ Embora interações aleatórias sejam admitidas como ingredientes fundamentais na maioria das abordagens, existem propostas de modelos sem desordem que exibem comportamento vítreo (Bouchaud e Mézard, 1994).
} 
recozidas (annealed) é de certa forma trivial ${ }^{8}$. O caso temperado (quenched), mais adequado para vidros de spins, possui dificuldades não apenas do ponto de vista analítico, mas numérico também.

Na abordagem de Sherrington e Kirkpatrick (SK), considera-se uma hamiltoniana de Edwards e Anderson simplificada, com spins do tipo Ising e interações de alcance infinito devidamente escaladas com o tamanho do sistema (Sherrington e Kirkpatrick, 1975; Kirkpatrick e Sherrington, 1978). As propriedades termodinâmicas podem ser obtidas de forma exata, embora não rigorosa, por meio do chamado método das réplicas, que detalharemos nas próximas seções. A solução admitida como correta para o modelo SK é a solução de Parisi com infinitas etapas de quebra de simetria entre réplicas ${ }^{9}$ (Parisi, 1979, 1980a, 1980b, 1980c). Apenas recentemente, com o trabalho de Talagrand (Talagrand, 2003), e importantes contribuições de Guerra e Toninelli (Guerra e Toninelli, 2002), tornou-se possível o estabelecimento de bases matemáticas (e termodinâmicas) firmes para a solução de Parisi.

\subsection{O problema físico}

O estudo do efeito da compressibilidade em sistemas magnéticos possui motivações diversas, tanto analíticas e numéricas quanto experimentais. Sob o ponto de vista teórico, é conhecido que o comportamento crítico de modelos de spin na rede pode ser alterado pela introdução de um termo de acoplamento magneto-mecânico na hamiltoniana. Os modelos de Ising ferromagnético (Domb, 1956; Salinas, 1974; Henriques e Salinas, 1987) e antiferromagnético (Chen e Kardar, 1986) são exemplos suficientemente abrangentes, onde o acoplamento entre os graus elásticos de liberdade e a interação magnética produz desde mudanças na ordem da transição (Henriques e Salinas, 1987) à remoção da degenerescência e emergência de um estado fundamental único com spins up e down distribuídos alternadamente em sistemas frustrados (Chen e Kardar, 1986).

Sob o ponto de vista experimental, a inserção de compressibilidade em um modelo

\footnotetext{
${ }^{8}$ Veja o Capítulo 1 para uma breve introdução aos tipos de desordem.

${ }^{9} \mathrm{~A}$ solução com simetria de réplicas dada por Sherrington e Kirkpatrick é instável à esquerda da linha de AlmeidaThouless (Almeida e Thouless, 1978).
} 
com ligações e campos aleatórios (Random bond-random field model) é necessária, por exemplo, para reproduzir a transição de primeira ordem entre as fases para e ferroelétrica, observada no cristal molecular misto $\mathrm{Rb}_{1-x}\left(\mathrm{NH}_{4}\right)_{x} \mathrm{H}_{2} \mathrm{AsO}_{4}$ (Papantopoulos et al., 1994) ${ }^{10}$. Estes resultados remontam a estudos anteriores de diagramas de fases de misturas de cristais ferro e antiferroelétricos da família KDP com ligações de hidrogênio (Courtens, 1983; Matsushita e Matsubara, 1985), que discutiremos mais tarde ${ }^{11}$. Outros cristais moleculares mistos, como o $\mathrm{K}(\mathrm{CN})_{x} \mathrm{Br}_{1-x}$, têm sido estudados com base em modelos orientacionais com desordem e graus de liberdade elásticos (Tadic, Pirc, Blinc, Petersson, e Wiotte, 1994).

Chamamos a atenção para o fato de que simulações numéricas com alta precisão de modelos compressíveis tornaram-se possíveis apenas recentemente, com o aumento da capacidade de processamento dos computadores modernos e o desenvolvimento de algoritmos mais eficazes (Mitchell, Pereira, e Landau, 2008). Aqui também servem de paradigma os modelos de Ising compressíveis com interações ferro (Landau, 2006) e antiferromagnéticas (Cannavacciuolo e Landau, 2005), sendo o método de Monte Carlo o tratamento típico empregado. Estudos semelhantes aplicados a sistemas desordenados são deveras mais escassos. Para vidros de spins, por exemplo, apesar dos resultados recentes obtidos a partir de simulações de Monte Carlo para o modelo de Edwards-Anderson compressível bidimensional (Marshall, Chacraborty, e Nagel, 2006; Marshall, 2007), não há relatos na literatura sobre estudos de modelos compressíveis a partir de abordagens analíticas ${ }^{12}$. Neste sentido, o presente trabalho visa cobrir esta lacuna.

\footnotetext{
${ }^{10} \mathrm{~A}$ transição do cristal de Rubídio puro, $\mathrm{RbH}_{2} \mathrm{AsO}_{4}$, ocorre a $T_{c}=110 \mathrm{~K}$ e é de primeira ordem, com uma descontinuidade da polarização. Para baixa desordem substitucional, $x<0.04$, a resposta dielétrica é semelhante à da amostra pura. Para $0.04<x<0.1$, medidas dielétricas apontam para uma coexistência de ordenamento ferroelétrico e de desordem vítrea para temperaturas abaixo de $T_{t r}<T_{c}$. O modelo com ligações e campos aleatórios rígido, i.e. sem graus de liberdade elásticos, prevê a existência de uma fase vítrea mesmo no limite de desordem muito baixa $x \rightarrow 0$. Com a introdução de compressibilidade, a transição para a fase ordenada, que era de segunda ordem no modelo rígido, passa a ser de primeira ordem, com uma descontinuidade da polarização e do parâmetro de ordem de Edwards e Anderson na temperatura crítica. ${ }^{11}$ Veja a seção 3.6 .

${ }^{12} \mathrm{~A}$ intersecção que existe entre as propriedades de vidros de spin e as propriedades ferroelétricas observadas em $\mathrm{Rb}_{1-x}\left(\mathrm{NH}_{4}\right)_{x} \mathrm{H}_{2} \mathrm{AsO}_{4}$ (Papantopoulos et al., 1994) não será abordada aqui.
} 


\subsection{Modelo de Sherrington-Kirkpatrick compressível}

A hamiltoniana do modelo de Sherrington-Kirkpatrick compressível (Sherrington e Kirkpatrick, 1975; Liarte et al., 2009) proposta neste trabalho é dada por

$$
\mathcal{H}=-\sum_{i<j} J_{i j}\left[1-\gamma\left(v-v_{0}\right)\right] S_{i} S_{j}-H \sum_{i} S_{i}+\frac{1}{2} k N\left(v-v_{0}\right)^{2}
$$

onde $H$ representa um campo magnético externo, $v$ o volume específico do sistema, $\gamma$ e $v_{0}$ são parâmetros positivos, $k>0$ é uma constante elástica e $S_{i}= \pm 1$, para todo $1 \leq i \leq N$. As variáveis $J_{i j}$ são aleatórias, independentes e identicamente distribuídas segundo uma distribuição normal de média $J_{0} / N$ e variância $J^{2} / N$, escaladas de forma a garantir o limite termodinâmico. O termo de acoplamento magneto-mecânico $\gamma$ é responsável por um aumento ou diminuição da interação entre dois spins $i$ e $j$, produzidos por uma variação da distância média entre $i$ e $j$ quando a diferença $\left(v-v_{0}\right)$ é alterada. O terceiro termo corresponde a uma energia de interação harmônica. Esse tipo de abordagem, com uma variável de volume específico global apenas, assemelha-se à proposta pioneira de Domb (Domb, 1956).

O ensemble das pressões se mostra mais conveniente do ponto de vista analítico para este sistema. Ademais, o uso de variáveis intensivas na análise dos resultados, a saber temperatura e pressão, possui um maior apelo experimental. A energia livre do sistema é, portanto, obtida da equação

$$
-\beta g=\lim _{N \rightarrow \infty} \frac{1}{N}\langle\ln Y\rangle,
$$

onde a função de partição $Y$ é dada por ${ }^{13}$

$$
Y=\int_{-\infty}^{\infty} d v \exp (-\beta p V) \operatorname{Tr} \exp (-\beta \mathcal{H})
$$

e $\langle\cdots\rangle$ denota uma média sobre a desordem ${ }^{14}$,

$$
\langle\cdots\rangle=\int_{-\infty}^{\infty} \prod_{i<j} \frac{d J_{i j}}{\sqrt{2 \pi} \sigma} \exp \left[-\sum_{i<j} \frac{\left(J_{i j}-\left\langle J_{i j}\right\rangle\right)^{2}}{2 \sigma^{2}}\right](\cdots),
$$

\footnotetext{
${ }^{13} \mathrm{O}$ intervalo de integração em $v$, a princípio $(0, \infty)$, pode ser estendido a $(-\infty, \infty)$, se forem consideradas pequenas oscilações em torno de $v_{0}$. A convergência é garantida pelo termo $k N\left(v-v_{0}\right)^{2} / 2$ da hamiltoniana.

${ }^{14}$ Note que (3.6) é válida desde que $g$ seja auto-mediante, ou seja, para todas as realizações da matriz de interação $\mathbb{J}$ (a menos de um conjunto de medida nula), $g\left(\left\{J_{i j}\right\}\right)=\left\langle g\left(\left\{J_{i j}\right\}\right)\right\rangle_{J}$.
} 
com

$$
\sigma^{2}=J^{2} / N, \quad\left\langle J_{i j}\right\rangle=J_{0} / N
$$

A integração no volume específico resulta

$$
Y=\exp \left[-\beta N\left(p v_{0}-\frac{p^{2}}{2 k}\right)\right] \operatorname{Tr} \exp \left(-\beta \mathcal{H}_{e f f}\right)
$$

onde a hamiltoniana efetiva

$$
\mathcal{H}_{e f f}=-H \sum_{i} S_{i}-\left(1+\frac{\gamma p}{k}\right) \sum_{i<j} J_{i j} S_{i} S_{j}-\frac{\gamma^{2}}{2 k N}\left(\sum_{i<j} J_{i j} S_{i} S_{j}\right)^{2},
$$

possui um termo de interação entre quatro spins, comum em modelos magnéticos compressíveis no ensemble das pressões. O termo quadrático em $J_{i j}$ pode ser linearizado por meio de uma transformação gaussiana,

$$
\begin{gathered}
\exp \left[\frac{\beta \gamma^{2}}{2 k N}\left(\sum_{i<j} J_{i j} S_{i} S_{j}\right)^{2}\right]=\sqrt{\frac{N \beta \gamma^{2} J^{2}}{2 \pi k}} \int_{-\infty}^{\infty} d x \exp \left(-\frac{N \beta \gamma^{2} J^{2}}{2 k} x^{2}\right. \\
\left.+\frac{\beta J \gamma^{2} x}{k} \sum_{i<j} J_{i j} S_{i} S_{j}\right)
\end{gathered}
$$

que implica

$$
\begin{aligned}
Y=\exp & {\left[-\beta N\left(p v_{0}-\frac{p^{2}}{2 k}\right)\right] \int_{-\infty}^{\infty} d x \exp \left(-\frac{N \beta \gamma^{2} J^{2}}{2 k} x^{2}\right) } \\
\times & \operatorname{Tr} \exp \left[\beta\left(1+\frac{\gamma p}{k}+\frac{J \gamma^{2} x}{k}\right) \sum_{i<j} J_{i j} S_{i} S_{j}+\beta H \sum_{i} S_{i}\right] .
\end{aligned}
$$

A despeito da integração em $x$ acima, que para todos os efeitos pode ser calculada ao final pelo método de Laplace, a função de partição no ensemble das pressões exibe uma forma similar à função de partição canônica do modelo SK rígido, o que sugere a aplicação do método das réplicas.

\subsection{Método das réplicas}

O método das réplicas se baseia na identidade logarítmica

$$
\langle\ln Y\rangle=\lim _{n \rightarrow 0} \frac{1}{n} \ln \left\langle Y^{n}\right\rangle .
$$

Para $n$ inteiro, $\left\langle Y^{n}\right\rangle$ é a função de partição de um sistema de $n$ réplicas independentes da hamiltoniana original, cuja determinação é padrão. A energia livre é obtida a posteriori a 
partir de uma continuação analítica em $n$, tomando-se o limite $n \rightarrow 0$. Discussões sobre o rigor matemático a respeito da continuação analítica em $n$ ou sobre a inversão na ordem dos limites em $N$ e $n$ estão além do objetivo deste trabalho ${ }^{15}$.

A função de partição de $n$ réplicas é dada por

$$
\begin{aligned}
\left\langle Y^{n}\right\rangle=\exp & {\left[-\beta N n\left(p v_{0}-\frac{p^{2}}{2 k}\right)\right] \int_{-\infty}^{\infty} \prod_{\alpha} d x^{\alpha} \exp \left[-\frac{N \beta \gamma^{2} J^{2}}{2 k} \sum_{\alpha}\left(x^{\alpha}\right)^{2}\right] } \\
\times & \left\langle\operatorname{Tr}_{n} \exp \left[\sum_{i<j} J_{i j}\left(\beta \sum_{\alpha} \xi^{\alpha} S_{i}^{\alpha} S_{j}^{\alpha}\right)+\beta H \sum_{i, \alpha} S_{i}^{\alpha}\right]\right\rangle
\end{aligned}
$$

onde $\xi^{\alpha}=1+\gamma p / k+J \gamma^{2} x^{\alpha} / k$. As médias sobre as realizações de $\mathbb{J}$ resultam então, descartando-se termos de ordem $N^{-1}$,

$$
\begin{aligned}
\left\langle Y^{n}\right\rangle=\exp & {\left[-\beta N n\left(p v_{0}-\frac{p^{2}}{2 k}\right)\right] \int_{-\infty}^{\infty} \prod_{\alpha} d x^{\alpha} \exp \left[-\frac{N \beta \gamma^{2} J^{2}}{2 k} \sum_{\alpha}\left(x^{\alpha}\right)^{2}\right] } \\
& \times \operatorname{Tr}_{n} \exp \left(\frac{\beta^{2} J^{2}}{2 N} \sum_{\alpha, \beta} \xi^{\alpha} \xi^{\beta} \sum_{i<j} S_{i}^{\alpha} S_{i}^{\beta} S_{j}^{\alpha} S_{j}^{\beta}+\frac{\beta J_{0}}{N} \sum_{\alpha} \xi^{\alpha} \sum_{i<j} S_{i}^{\alpha} S_{j}^{\alpha}\right. \\
& \left.+\beta H \sum_{i, \alpha} S_{i}^{\alpha}\right)
\end{aligned}
$$

ou ainda, tendo em vista as relações

$$
\begin{aligned}
\sum_{i<j} S_{i}^{\alpha} S_{i}^{\beta} S_{j}^{\alpha} S_{j}^{\beta} & =\frac{1}{2}\left(\sum_{i} S_{i}^{\alpha} S_{i}^{\beta}\right)^{2}-\frac{N}{2} \\
\sum_{i<j} S_{i}^{\alpha} S_{j}^{\alpha} & =\frac{1}{2}\left(\sum_{i} S_{i}^{\alpha}\right)^{2}-\frac{N}{2} \\
\sum_{\alpha, \beta} & =2 \sum_{\alpha<\beta}+\sum_{\alpha=\beta},
\end{aligned}
$$

obtemos

$$
\begin{aligned}
\left\langle Y^{n}\right\rangle=\exp & {\left[-\beta N n\left(p v_{0}-\frac{p^{2}}{2 k}\right)\right] \int_{-\infty}^{\infty} \prod_{\alpha} d x^{\alpha} \exp \left[-\frac{N \beta \gamma^{2} J^{2}}{2 k} \sum_{\alpha}\left(x^{\alpha}\right)^{2}\right.} \\
+ & \left.\frac{\beta^{2} J^{2} N}{4} \sum_{\alpha}\left(\xi^{\alpha}\right)^{2}\right] \operatorname{Tr}_{n} \exp \left[\frac{\beta^{2} J^{2}}{2 N} \sum_{\alpha<\beta} \xi^{\alpha} \xi^{\beta}\left(\sum_{i} S_{i}^{\alpha} S_{i}^{\beta}\right)^{2}\right. \\
+ & \left.\frac{\beta J_{0}}{2 N} \sum_{\alpha} \xi^{\alpha}\left(\sum_{i} S_{i}^{\alpha}\right)^{2}+\beta H \sum_{i, \alpha} S_{i}^{\alpha}\right] .
\end{aligned}
$$

\footnotetext{
${ }^{15}$ Para o modelo rígido, veja por exemplo (Hemmen e Palmer, 1979) para uma discussão sobre a inversão dos limites, e (Binder e Young, 1986) para discussões gerais.
} 
Podemos simplificar a expressão para o traço por meio de identidades gaussianas, introduzindo as funções magnetização $\left\{m^{\alpha}\right\}$ e de overlap entre réplicas $\left\{q^{\alpha \beta}\right\}$ segundo as equações

$$
\begin{aligned}
\exp \left[\frac{\beta^{2} J^{2}}{2 N} \xi^{\alpha} \xi^{\beta}\left(\sum_{i} S_{i}^{\alpha} S_{i}^{\beta}\right)^{2}\right]= & \sqrt{\frac{N \beta^{2} J^{2} \xi^{\alpha} \xi^{\beta}}{2 \pi} \int_{-\infty}^{\infty} d q^{\alpha \beta} \exp \left[-\frac{N \beta^{2} J^{2}}{2} \xi^{\alpha} \xi^{\beta}\left(q^{\alpha \beta}\right)^{2}\right.} \\
& \left.+\beta^{2} J^{2} \xi^{\alpha} \xi^{\beta} \sum_{i} S_{i}^{\alpha} S_{i}^{\beta} q^{\alpha \beta}\right], \\
\exp \left[\frac{\beta J_{0}}{2 N} \xi^{\alpha}\left(\sum_{i} S_{i}^{\alpha}\right)^{2}\right]= & \sqrt{\frac{N \beta J_{0} \xi^{\alpha}}{2 \pi}} \int_{-\infty}^{\infty} d m^{\alpha} \exp \left[-\frac{N \beta J_{0}}{2} \xi^{\alpha}\left(m^{\alpha}\right)^{2}\right. \\
& \left.+\beta J_{0} \xi^{\alpha} \sum_{i} S_{i}^{\alpha} m^{\alpha}\right],
\end{aligned}
$$

de forma que

$$
\left\langle Y^{n}\right\rangle=\int_{-\infty}^{\infty} \prod_{\alpha} d x^{\alpha} \int_{-\infty}^{\infty} \prod_{\alpha} d m^{\alpha} \int_{-\infty}^{\infty} \prod_{\alpha<\beta} d q^{\alpha \beta} \exp \left[N G\left(x^{\alpha}, m^{\alpha}, q^{\alpha \beta}\right)\right],
$$

$\operatorname{com} G=G\left(x^{\alpha}, m^{\alpha}, q^{\alpha \beta}\right)$ dado por

$$
\begin{aligned}
G=- & \beta n\left(p v_{0}-\frac{p^{2}}{2 k}\right)-\frac{\beta \gamma^{2} J^{2}}{2 k} \sum_{\alpha}\left(x^{\alpha}\right)^{2}+\frac{\beta^{2} J^{2}}{4} \sum_{\alpha}\left(\xi^{\alpha}\right)^{2}-\frac{\beta J_{0}}{2} \sum_{\alpha} \xi^{\alpha}\left(m^{\alpha}\right)^{2} \\
& -\frac{\beta^{2} J^{2}}{2} \sum_{\alpha<\beta} \xi^{\alpha} \xi^{\beta}\left(q^{\alpha \beta}\right)^{2}+\ln \operatorname{Tr} \exp \left(\beta^{2} J^{2} \sum_{\alpha<\beta} \xi^{\alpha} \xi^{\beta} q^{\alpha \beta} S^{\alpha} S^{\beta}\right. \\
& \left.+\beta J_{0} \sum_{\alpha} \xi^{\alpha} m^{\alpha} S^{\alpha}+\beta H \sum_{\alpha} S^{\alpha}\right) .
\end{aligned}
$$

No limite termodinâmico, a aplicação do método de Laplace leva à expressão para a energia livre

$$
\begin{aligned}
-\beta g=- & \beta\left(p v_{0}-\frac{p^{2}}{2 k}\right)+\lim _{n \rightarrow 0} \frac{1}{n}\left[-\frac{\beta \gamma^{2} J^{2}}{2 k} \sum_{\alpha}\left(x^{\alpha}\right)^{2}+\frac{\beta^{2} J^{2}}{4} \sum_{\alpha}\left(\xi^{\alpha}\right)^{2}\right. \\
& -\frac{\beta^{2} J^{2}}{2} \sum_{\alpha<\beta} \xi^{\alpha} \xi^{\beta}\left(q^{\alpha \beta}\right)^{2}-\frac{\beta J_{0}}{2} \sum_{\alpha} \xi^{\alpha}\left(m^{\alpha}\right)^{2}+\ln \operatorname{Tr} \\
& \left.\times \exp \left(\beta^{2} J^{2} \sum_{\alpha<\beta} \xi^{\alpha} \xi^{\beta} q^{\alpha \beta} S^{\alpha} S^{\beta}+\beta J_{0} \sum_{\alpha} \xi^{\alpha} m^{\alpha} S^{\alpha}+\beta H \sum_{\alpha} S^{\alpha}\right)\right]
\end{aligned}
$$

onde os conjuntos $\left\{x^{\alpha}\right\},\left\{q^{\alpha \beta}\right\}$ e $\left\{m^{\alpha}\right\}$ são tais que $G\left(x^{\alpha}, m^{\alpha}, q^{\alpha \beta}\right)$ é máximo. Verifica-se imediatamente que, para garantir a existência de um limite termodinâmico bem-definido, 
as condições $m^{\alpha}=m$ e $x^{\alpha}=x$, para todo $\alpha$, devem ser satisfeitas. Além disto, a escolha da forma da parametrização de $q^{\alpha \beta}$ também deve satisfazer um conjunto de exigências termodinâmicas (Binder e Young, 1986). Acredita-se que a descrição correta da fase vidro de spin do modelo SK seja dada pela solução de Parisi com um número infinito de etapas de quebra de simetria entre réplicas. Esta solução é, no entanto, difícil de ser analisada analítica e numericamente. A solução com simetria de réplicas proposta por Sherrington e Kirkpatrick, apesar de instável em vasta região do diagrama de fases, mostra-se suficiente para os propósitos abordados neste estudo.

\subsection{Solução com simetria de réplicas e análise do comporta- mento crítico}

Segundo o Ansatz de Sherrington e Kirkpatrick $\left(q^{\alpha \beta}=q, m^{\alpha}=m\right.$ e $\left.x^{\alpha}=x \forall \alpha, \beta\right)$, a energia livre no ensemble das pressões fica dada por

$$
\begin{aligned}
-\beta g=- & \beta\left(p v_{0}-\frac{p^{2}}{2 k}\right)-\frac{\beta \gamma^{2} J^{2}}{2 k} x^{2}+\frac{\beta^{2} J^{2}}{4} \xi^{2}(1-q)^{2}-\frac{\beta J_{0} \xi}{2} m^{2} \\
& +\int_{-\infty}^{\infty} \frac{d z}{\sqrt{2 \pi}} \exp \left(-\frac{z^{2}}{2}\right) \ln 2 \cosh \left[\beta\left(J \xi q^{1 / 2} z+J_{0} \xi m+H\right)\right],
\end{aligned}
$$

com

$$
\xi=1+\frac{\gamma p}{k}+\frac{J \gamma^{2} x}{k}
$$

e onde $m, q$ e $x$ obedecem às condições de extremo,

$$
\begin{gathered}
q=\int_{-\infty}^{\infty} \frac{d z}{\sqrt{2 \pi}} \exp \left(-\frac{z^{2}}{2}\right) \tanh ^{2}\left[\beta\left(J \xi q^{1 / 2} z+J_{0} \xi m+H\right)\right], \\
m=\int_{-\infty}^{\infty} \frac{d z}{\sqrt{2 \pi}} \exp \left(-\frac{z^{2}}{2}\right) \tanh \left[\beta\left(J \xi q^{1 / 2} z+J_{0} \xi m+H\right)\right], \\
x=\frac{\beta J}{2} \xi\left(1-q^{2}\right)+\frac{J_{0}}{2 J} m^{2} .
\end{gathered}
$$

A campo nulo, expansões das equações de estado (3.28), (3.29), (3.30) e algumas manipulações algébricas determinam as fronteiras entre as diversas fases do sistema. Nas fases vidro de spins, ferromagnética e paramagnética tem-se, respectivamente, $q \neq 0$ e 
$m=0, q \neq 0$ e $m \neq 0, q=m=0^{16}$. A linha de transição entre as fases vidro de spins e paramagnética é obtida expandindo-se as equações (3.28) e (3.30) para $m=0$, que admitem solução $q>0$ para

$$
1-\frac{1}{\xi \beta J}>0
$$

e, portanto,

$$
t_{c 1}=1+\frac{\gamma p}{k}+\frac{J \gamma^{2}}{2 k}
$$

onde $t=k_{B} T / J$ e $t_{c 1}$ é a temperatura crítica escalada.

A linha de transição entre as fases ferro e paramagnética provém das expansões das equações (3.28), (3.29) e (3.30), desprezando-se termos de ordem $\mathrm{O}\left(m^{4}, q^{2}\right)$. Segue de $(3.30)$,

$$
x=\frac{\beta J}{2} \xi+\frac{J_{0}}{2 J} m^{2}+\mathrm{O}\left(m^{4}, q^{2}\right)
$$

ou ainda, introduzindo-se variáveis adimensionais apropriadas,

$$
\begin{gathered}
t=\frac{k_{B} T}{J}, \quad j=\frac{J_{0}}{J}, \quad a=1+\frac{\gamma p}{k}, \quad b=\frac{J \gamma^{2}}{2 k} ; \\
\xi\left(1-\frac{b}{t}\right)=a\left(1+\frac{j b}{a} m^{2}\right)+\mathrm{O}\left(m^{4}, q^{2}\right) .
\end{gathered}
$$

Assim,

$$
\begin{aligned}
& q=\int_{-\infty}^{\infty} \frac{d z}{\sqrt{2 \pi}} \exp \left(-\frac{z^{2}}{2}\right) \tanh ^{2}\left[\frac{a}{t-b}\left(1+\frac{j b}{a} m^{2}\right)\left(q^{1 / 2} z+j m\right)\right], \\
& m=\int_{-\infty}^{\infty} \frac{d z}{\sqrt{2 \pi}} \exp \left(-\frac{z^{2}}{2}\right) \tanh \left[\frac{a}{t-b}\left(1+\frac{j b}{a} m^{2}\right)\left(q^{1 / 2} z+j m\right)\right],
\end{aligned}
$$

de onde segue,

$$
\begin{gathered}
q=\left(\frac{a}{t-b}\right)^{2}\left(q+j^{2} m^{2}\right)+\mathrm{O}\left(m^{4}, q^{2}\right) \\
1=\frac{a j}{t-b}\left(1+\frac{j b}{a} m^{2}\right)-\frac{1}{3}\left(\frac{a}{t-b}\right)^{3}\left(j^{3} m^{2}+3 q j\right)+\mathrm{O}\left(m^{3}, q^{3 / 2}\right)
\end{gathered}
$$

\footnotetext{
${ }^{16}$ Note que a fase vidro de spin é definida de forma "rudimentar" aqui, tomando-se partido do significado físico de $q^{\alpha \beta}$, $q^{\alpha \beta}=\left\langle\left\langle S_{i}\right\rangle_{T}^{2}\right\rangle$, onde $\langle\cdots\rangle_{T}$ denota uma média térmica usual. Em uma definição mais precisa, a fase vidro de spin deve estar presente na região onde há quebra de simetria na função de overlap entre réplicas.
} 
que resulta na expressão para a temperatura de transição, no limite $q, m \rightarrow 0$,

$$
t_{c 2}=\left(1+\frac{\gamma p}{k}\right) j+\frac{J \gamma^{2}}{2 k}
$$

Para estudar a transição entre as fases ferromagnética e vidro de spins, analisaremos o comportamento da susceptibilidade magnética $\chi$ a campo nulo no limite $m \rightarrow 0$. A equação (3.29) pode ser reescrita na forma

$$
m=\int \frac{d z}{\sqrt{2 \pi}} \exp \left(-\frac{z^{2}}{2}\right) \tanh \left[\frac{\left(a+b j m^{2}\right)}{t-b\left(1-q^{2}\right)}\left(q^{1 / 2} z+j m\right)+\frac{1}{t} \frac{H}{J}\right],
$$

de onde segue

$$
\begin{aligned}
\chi=\int & \frac{d z}{\sqrt{2 \pi}} \exp \left(-\frac{z^{2}}{2}\right)\left\{1-\tanh ^{2}\left[\frac{\left(a+b j m^{2}\right)}{t-b\left(1-q^{2}\right)}\left(q^{1 / 2} z+j m\right)+\frac{1}{t} \frac{H}{J}\right]\right\} \\
\times & \left\{\chi\left[\frac{2 b j m}{t-b\left(1-q^{2}\right)}\left(q^{1 / 2} z+j m\right)+j \frac{\left(a+b j m^{2}\right)}{t-b\left(1-q^{2}\right)}\right]-\frac{\partial q}{\partial H}\left(a+b j m^{2}\right)\right. \\
\times & {\left.\left[2 b q \frac{q^{1 / 2} z+j m}{\left(t-b\left(1-q^{2}\right)\right)^{2}}+\frac{1}{2} \frac{q^{-1 / 2} z}{t-b\left(1-q^{2}\right)}\right]+\frac{1}{t J}\right\} }
\end{aligned}
$$

ou ainda, para $H=0$,

$$
\chi=(1-q)\left\{\chi \frac{a j+3 b j^{2} m^{2}}{t-b\left(1-q^{2}\right)}-2 b q j m \frac{\partial q}{\partial H} \frac{a+b j m^{2}}{\left[t-b\left(1-q^{2}\right)\right]^{2}}+\frac{1}{t J}\right\} .
$$

No limite $m \rightarrow 0$,

$$
J \chi=\frac{(1-q)\left[t-b\left(1-q^{2}\right)\right]}{t\left[t-b\left(1-q^{2}\right)-a j(1-q)\right]} .
$$

A transição ocorre nos pontos onde a susceptibilidade diverge, i.e., para cada $t, j$ é obtido como solução do conjunto de equações

$$
\begin{gathered}
t-b\left(1-q^{2}\right)-a j(1-q)=0 \\
q=\int \frac{d z}{\sqrt{2 \pi}} \exp \left(-\frac{z^{2}}{2}\right) \tanh ^{2}\left[\frac{a q^{1 / 2} z}{t-b\left(1-q^{2}\right)}\right] .
\end{gathered}
$$

Expansões das equações (3.28), (3.29) e (3.30) em ordens superiores de $m$ e $q$ resultam

$$
\begin{gathered}
m=\frac{j a m}{t-b}\left(1+\frac{b}{a} j m^{2}\right)-\frac{1}{3} \frac{j^{3} a^{3} m}{(t-b)^{3}}\left(m^{2}+3 q j^{-2}+3 \frac{b}{a} j m^{4}+9 \frac{b}{a} j^{-1} q m^{2}\right) \\
+\mathrm{O}\left(m^{6}, q^{3}\right) \\
q=\left(\frac{j a}{t-b}\right)^{2}\left(1+2 \frac{b}{a} j m^{2}\right)\left(q j^{-2}+m^{2}\right)-\frac{2}{3}\left(\frac{j a}{t-b}\right)^{4}\left(3 q^{2} j^{-4}+m^{4}+6 q j^{-2} m^{2}\right) \\
\quad+\mathrm{O}\left(m^{6}, q^{3}\right) .
\end{gathered}
$$


Para $m=0$, os coeficientes das potências de $q$ demonstram que a introdução de um termo de acoplamento magneto-mecânico não induz nenhuma mudança na ordem da transição entre as fases vidro de spin e paramagnética. A emergência de um ponto tricrítico ocorre apenas na transição entre as fases ferro e paramagnética, e é assinalada por uma mudança no sinal do coeficiente da potência de ordem três de $m$. Após algumas manipulações algébricas é possível obter

$$
p_{t c}=\frac{3 J \gamma j\left(j^{2}-1\right)}{2\left(j^{2}+2\right)}-\frac{k}{\gamma}
$$

para a pressão no ponto tricrítico. A temperatura é dada pela equação (3.40). Note que a exigência $p_{t c}>0$ só é satisfeita para distribuições suficientemente "estreitas",

$$
J^{2}<\frac{J_{0}^{2}+\frac{4 k J_{0}}{3 \gamma^{2}}}{1+\frac{4 k}{3 J_{0} \gamma^{2}}} .
$$

Apresentamos na Figura 3.4 gráficos de $q^{1 / 2}$ e $m$ como função da temperatura para $a=1.5$ e $b=0.5$. Para estes parâmetros o ponto tricrítico está localizado em $j=2$. A transição é descontínua para pontos acima do ponto tricrítico (gráfico à direita), e contínua para pontos abaixo (gráfico à esquerda).
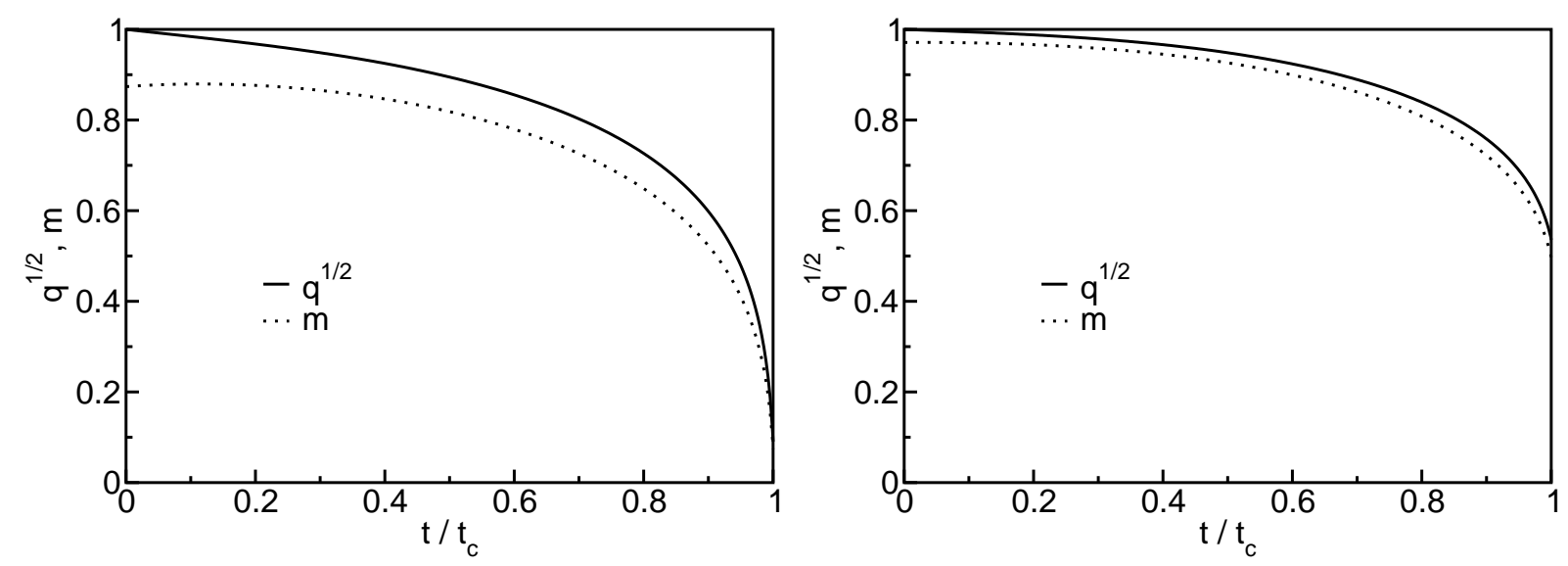

Figura 3.4: Gráficos de $q^{1 / 2}$ e $m$ como função da temperatura para $a=1.5, b=0.5, j=1.75$ (à esquerda) e $j=2.25$ (à direita).

Por completeza, apresentamos no Apêndice A a análise da estabilidade da solução com simetria de réplicas do modelo SK compressível. Os cálculos seguem ipsis litteris o 
trabalho de Almeida e Thouless (Almeida e Thouless, 1978). O acoplamento magnetomecânico, neste caso, induz apenas um deslocamento na linha de estabilidade, que é dada por

$$
\left(\frac{k_{B} T}{J}\right)^{2}=(a+2 b x)^{2} \int_{-\infty}^{\infty} \frac{d z}{\sqrt{2 \pi}} \exp \left(-\frac{z^{2}}{2}\right) \operatorname{sech}^{4}\left[\beta(a+2 b x)\left(J q^{1 / 2} z+J_{0} m\right)\right]
$$

onde $q, m$ e $x$ são obtidos das condições de extremo (3.28), (3.29) e (3.30). O diagrama de fases do modelo SK compressível, com a linha de Almeida e Thouless, será apresentado na próxima seção, onde discutiremos também possíveis conexões com resultados experimentais e perspectivas futuras de trabalho.

\subsection{Diagrama de fases e considerações finais}

O diagrama de fases do modelo SK compressível é apresentado na Figura 3.5, com $j=J_{0} / J$ na abscissa e $t=k_{B} T / J$ na ordenada. Os parâmetros $\gamma, p$ e $k$ são escolhidos tais que $a=1.5$ e $b=0.5$. As linhas pontilhadas representam as fronteiras de fase do modelo SK rígido. As linhas (1), (2) e (4) representam as fronteiras entre as fases vidro de spins e paramagnética, ferro e paramagnética, vidro de spins e ferromagnética, respectivamente. A linha (3) representa a transição de primeira ordem entre as fases ferro e paramagnética e a linha (5) delimita a fronteira de estabilidade da solução com simetria de réplicas. Para esses valores de $a$ e $b$, o ponto tricrítico (ponto de encontro entre as linhas (2) e (3) no gráfico) está localizado em $j=2$.

A inserção de um termo de acoplamento magneto-mecânico desloca as fronteiras da fase vidro de spins, assim como a linha de Almeida-Thouless. Além disto, a linha de transição de segunda ordem entre as fases ferro e paramagnética termina em um ponto tricrítico, a partir do qual a transição passa a ser descontínua. A expressão para a energia livre (3.25) não indica nenhuma mudança significativa ${ }^{17}$ no diagrama de fases além das já esperadas se considerarmos mais etapas de quebra de simetria entre réplicas. É importante ressaltar que as conclusões permanecem inalteradas para outros modelos de campo médio, como o modelo de Ising com campos aleatórios e o modelo de vidro de spins esférico ferromagnético com interação de $p$-spins.

\footnotetext{
${ }^{17}$ Isto é, a emergência de um ponto tricrítico.
} 


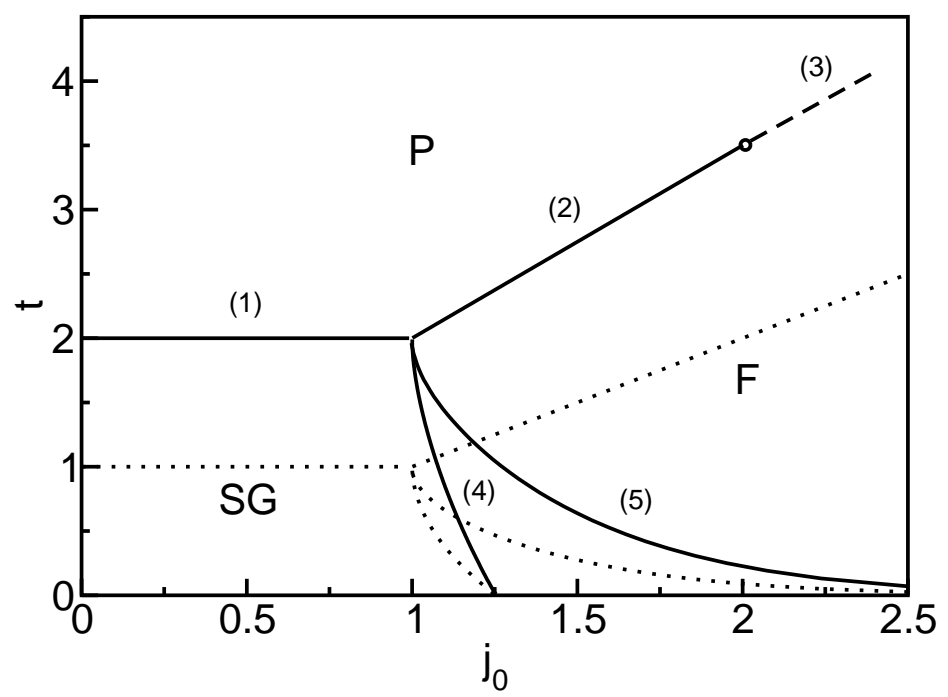

Figura 3.5: Diagrama de fases $J_{0} / J \times k_{B} T / J$ do modelo de Sherrington-Kirkpatrick compressível a campo nulo.

Recapitularemos agora alguns resultados experimentais para misturas de cristais ferro e antiferroelétricos com ligações de hidrogênio. Cristais de rubídio ${ }^{18}$, como $\mathrm{RbH}_{2} \mathrm{PO}_{4}$ (RDP), apresentam uma transição de segunda ordem para uma fase ferroelétrica a uma temperatura de aproximadamente $150 \mathrm{~K}$. Cristais de amônia, como $\mathrm{NH}_{4} \mathrm{H}_{2} \mathrm{PO}_{4}(\mathrm{ADP})$, por outro lado, exibem uma transição de primeira ordem para uma fase antiferroelétrica. A baixas temperaturas, dependendo da concentração $x$, uma fase vítrea pode surgir para a mistura destes cristais, $\mathrm{Rb}_{1-x}\left(\mathrm{NH}_{4}\right)_{x} \mathrm{H}_{2} \mathrm{PO}_{4}$ (RADP) (Matsushita e Matsubara, 1985; Courtens, 1983). Acreditamos que a introdução de um modelo de Sherrington-Kirkpatrick compressível em duas sub-redes possa reproduzir qualitativamente estes resultados.

Versões do modelo SK em duas sub-redes tem sido propostas para o estudo de transições de fases de materiais antiferromagnéticos com comportamento vítreo (Korenblit e Shender, 1985; Fyodorov, Korenblit, e Shender, 1987; Vieira, Nobre, e Yokoi, 2000; Liarte e Yokoi, 2008). Podemos definir a hamiltoniana de um modelo de Sherrington-Kirkpatrick compressível em duas sub-redes segundo a equação

$$
\mathcal{H}=-\sum_{i \in A, j \in B} J_{i j}\left[1-\gamma\left(v-v_{0}\right)\right] S_{i} S_{j}+\frac{1}{2} k N\left(v-v_{0}\right)^{2}
$$

\footnotetext{
${ }^{18}$ Note que fazemos referência a um cristal levemente diferente do citado anteriormente na seção 3.2, onde o arsênio ocupara o lugar do fósforo na composição.
} 
onde a soma é sobre os pares distintos de spins pertencentes a cada sub-rede, $A$ e $B$. Novamente, $J_{i j}$ são variáveis aleatórias independentes e identicamente distribuídas segundo uma distribuição normal com média $J_{0} / N$ e variância $J^{2} / N$, onde consideramos

$$
J_{0}=x J_{R}-(1-x) J_{A}
$$

sendo $J_{R}$ e $J_{A}$ parâmetros positivos, e tomamos $J^{2} \rightarrow J^{2} x(1-x)$, de forma que o limite $x=0(x=1)$ simula a transição ferroelétrica (antiferroelétrica). Com uma escolha apropriada dos parâmetros do modelo, os resultados obtidos no presente estudo sugerem um diagrama de fases típico conforme apresentado na Figura 3.6. As abreviaturas PE, FE, AFE e SG denotam as fases paraelétrica, ferroelétrica, antiferroelétrica e vítrea, respectivamente.

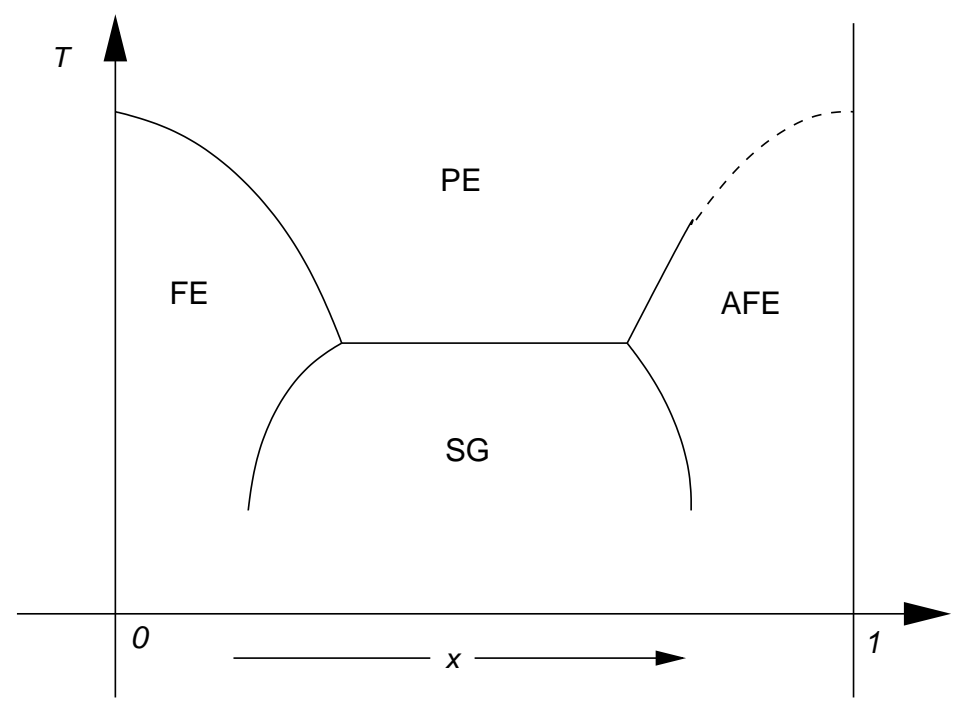

Figura 3.6: Diagrama de fases $x \times T$ de um modelo de Sherrington-Kirkpatrick compressível a campo nulo em duas sub-redes.

Trabalhos anteriores apresentam diagramas similares para a mistura RADP, obtidos a partir de cálculos de clusters para um modelo de Ising de pseudo-spins (Matsushita e Matsubara, 1985; Courtens, 1983). Mais recentemente, as propriedades de uma mistura semelhante, $\mathrm{Rb}_{1-x}\left(\mathrm{NH}_{4}\right)_{x} \mathrm{H}_{2} \mathrm{AsO}_{4}$ (RADA), foram investigadas com base em um modelo de Ising de pseudo-spins com ligações e campos aleatórios e a inclusão de graus de liberdade elásticos. Com um modelo simples como o modelo SK compressível, mostramos que é possível obter boa concordância qualitativa com os resultados experimentais para 
misturas de cristais ferro e antiferroelétricos com ligações de hidrogênio. A transição de primeira ordem entre as fases antiferro e paraelétrica emerge naturalmente da adição de graus de liberdade elásticos ao modelo SK. Enfatizamos que a inclusão de campos aleatórios gaussianos não é capaz de explicar a transição de primeira ordem observada nesses sistemas (Vieira et al., 2000; Soares, Nobre, e Almeida, 1994), que poderia estar associada ainda, além do efeito de graus de liberdade elásticos, a distribuições bimodais de campos aleatórios (Vieira et al., 2000; Nogueira, Nobre, Costa, e Coutinho, 1998), ou a considerações de modelos de vidros quadrupolares discretos e modelos de vidros de Potts (Binder e Reger, 1992). 



\section{Capítulo 4}

\section{Ordenamento nemático biaxial em}

\section{misturas de discos e cilindros}

Em meio a tantos casos de mal-uso de jargões da física no vocabulário cotidiano ${ }^{1}$, o termo cristais líquidos parece um exemplo de significado bastante intuitivo. Esse termo em geral se refere a materiais orgânicos com propriedades mecânicas e de simetria situadas entre as de um sólido cristalino e um líquido amorfo (Gennes e Prost, 1993). Como num cristal, algumas de suas propriedades exibem alto grau de anisotropia, ainda que a ausência de ordenamento translacional seja comparada à de um líquido ordinário (Chandrasekhar, 1992). Tais fases intermediárias (mesofases) e suas transições tem aplicações bastante variadas. Para ter uma ideia, a sigla LCD (Liquid Crystal Display) rotula uma tecnologia presente nas telas de boa parcela dos celulares, monitores e televisores atuais. Sob o ponto de vista teórico, há uma citação de de Gennes, ainda bastante atual, que expressa bem o estado da arte sobre as abordagens teóricas para os cristais líquidos nemáticos, que constituem provavelmente a classe mais simples dos cristais líquidos: "the statistical mechanics of liquids is difficult; the statistical mechanics of nematics is still worse!" (Gennes e Prost, 1993).

Neste capítulo estudamos a estabilidade da fase nemática biaxial para um modelo estatístico de cristais líquidos. Consideramos um modelo de Maier-Saupe com variáveis aleatórias de desordem que representam uma mistura binária de moléculas de formas

\footnotetext{
${ }^{1}$ Como energia e física quântica, por exemplo.
} 
cilíndrica e discótica. Empregamos um formalismo termodinâmico, fora do equilíbrio, definido em duas temperaturas numa situação de recozimento parcial $^{2}$, para mostrar que um pequeno afastamento da situação de completa termalização é suficiente para levar à estabilidade de uma fase nemática biaxial (Carmo et al., 2010). Na seção 4.1 fazemos uma breve revisão sobre cristais líquidos nemáticos. Na seção 4.2 discutimos as motivações físicas para estudar a estabilidade da fase nemática biaxial. A formulação em duas temperaturas é apresentada na seção 4.3 e o modelo de Maier-Saupe-Zwanzig é definido na seção 4.4. Discussões dos resultados com base na teoria de Landau-De Gennes são apresentadas na seção 4.5. Finalmente, algumas considerações são apresentadas na seção 4.6.

\subsection{Cristais líquidos nemáticos}

O ingrediente essencial para a formação de mesofases em cristais líquidos é a existência de uma forma geométrica anisotrópica, como discos ou cilindros, das moléculas ou agregados moleculares que constituem o material (Chandrasekhar, 1992). Chamamos cristais líquidos termotrópicos aqueles cujos elementos anisotrópicos (mesógenos) são moléculas (Figura 4.1a); e cristais líquidos liotrópicos cujos mesógenos são agregados de moléculas (Figura 4.1b) (Figueiredo-Neto e Salinas, 2005), geralmente constituídos por moléculas anfifílicas ${ }^{3}$. Cristais líquidos em forma cilíndrica são também usualmente classificados em três grupos: nemáticos, colestéricos e esméticos. Vamos nos concentrar apenas nos nemáticos, que possuem um alto grau de ordenamento orientacional, mas não translacional de longo alcance.

Em cristais líquidos nemáticos, o ordenamento paralelo dos vetores diretores associados aos mesógenos é favorecido tanto por interações de volume excluído quanto por interações de Van der Waals. No primeiro caso, mesógenos podem se mover com mais liberdade sem se sobrepor quando ordenados paralelamente, mesmo a altas concentrações, caracterizando uma desordem translacional que aumenta a entropia e, portanto, diminui a energia livre (Figura 4.2a). No caso de interações de Van der Waals, flutuações na polarização $\boldsymbol{p}$

\footnotetext{
${ }^{2}$ Partial annealing.

${ }^{3}$ Essas moléculas tendem a se agrupar quando em solução aquosa devido à sua estrutura, geralmente composta por uma cabeça polar e uma "cauda hidrofóbica".
} 
a)
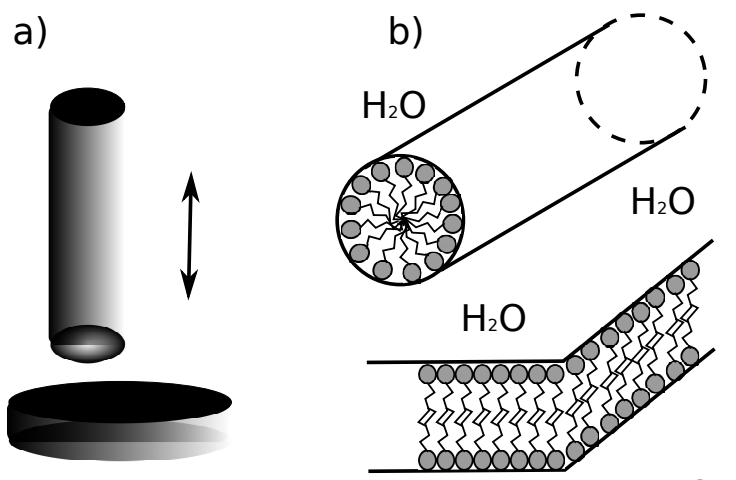

$\mathrm{H}_{2} \mathrm{O}$

Figura 4.1: a) Forma anisotrópica cilíndrica e discótica atribuída aos mesógenos. b) Ilustração de agregados moleculares anfifílicos presentes na composição de cristais líquidos liotrópicos.

de um mesógeno induzem polarizações $\boldsymbol{p}^{\prime}$ de sentido inverso em mesógenos próximos, que se tornam maiores se a orientação for paralela ${ }^{4}$ (Figura 4.2a). Uma maneira de descrever teoricamente o ordenamento orientacional de cristais líquidos nemáticos consiste em associar a cada mesógeno um vetor diretor local $\boldsymbol{n}_{i}$, que faz um ângulo $x_{i}$ com a direção definida por um vetor diretor global $\boldsymbol{N}$ (Figura 4.2b). A grandeza

$$
Q=\left\langle P_{2}\left(\cos x_{i}\right)\right\rangle=\frac{1}{2}\left\langle 3 \cos ^{2} x_{i}-1\right\rangle,
$$

onde $\langle\cdot\rangle$ denota uma média térmica, tem valor não nulo na fase ordenada e nulo na fase desordenada. Experimentalmente, cristais líquidos nemáticos apresentam uma transição de primeira ordem entre a chamada fase nemática (ordenada) para a fase isotrópica (desordenada orientacionalmente), em $T=T_{\mathrm{NI}}$ (Figura 4.2c).

Vamos escrever a equação (4.1) numa notação vetorial, independente de um sistema de coordenadas. Assim, seja $\boldsymbol{N}$ o vetor diretor global e $\boldsymbol{n}_{i}$ os diretores locais,

$$
\begin{aligned}
Q & =\left\langle\frac{1}{2}\left[3\left(\boldsymbol{n}_{i} \cdot \boldsymbol{N}\right)^{2}-1\right]\right\rangle \\
& =\left\langle\frac{1}{2}\left[3 \boldsymbol{N} \cdot\left(\boldsymbol{n}_{i} \boldsymbol{n}_{i}\right) \cdot \boldsymbol{N}-\boldsymbol{N} \cdot \boldsymbol{\delta} \cdot \boldsymbol{N}\right]\right\rangle,
\end{aligned}
$$

onde $\boldsymbol{\delta}$ é uma matriz cujos elementos são deltas de Kronecker $\left(\delta_{\mu \nu}\right)$ numa dada representação. O termo $\boldsymbol{n}_{i} \boldsymbol{n}_{i}$ denota um produto exterior de dois vetores, com componentes $n_{i}^{\mu} n_{i}^{\nu}$, sendo $n_{i}^{\mu}$ as componentes do vetor $\boldsymbol{n}_{i}$ na mesma representação. Usamos $|\boldsymbol{N}|=1$

\footnotetext{
${ }^{4}$ Veja o Capítulo 2 de (Warner e Terentjev, 2003).
} 
a)
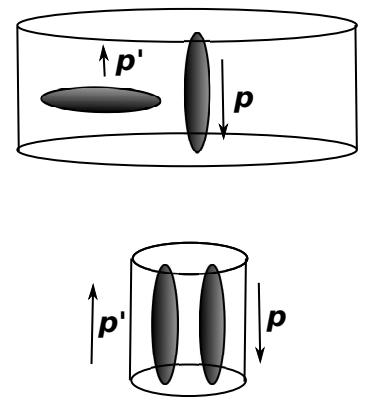

b)

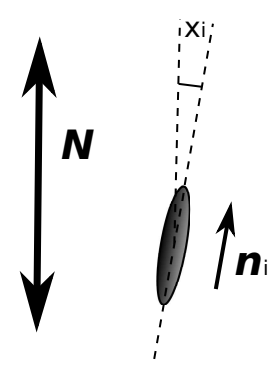

c) Nemática

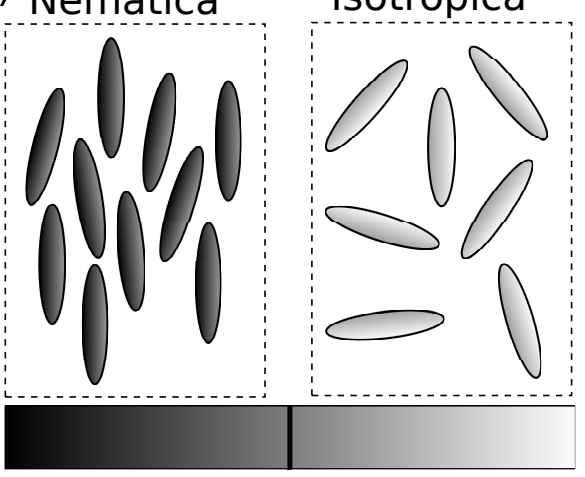

Temperatura $->\mathrm{T}_{\mathrm{NI}}$

Figura 4.2: a) O ordenamento orientacional é favorecido tanto por interações estéricas de volume excluído quando interações de Van der Waals. b) Descrição do parâmetro de ordem nemático. c) Ilustração da transição entre as fases nemática e isotrópica em $T=T_{\mathrm{NI}}$.

na equação (4.2). Podemos ainda escrever

$$
Q=\boldsymbol{N} \cdot\left\langle\frac{1}{2}\left(3 \boldsymbol{n}_{i} \boldsymbol{n}_{i}-\boldsymbol{\delta}\right)\right\rangle \cdot \boldsymbol{N}
$$

Uma vez que a escolha de $\boldsymbol{N}$ é arbitrária, a equação (4.3) sugere que o parâmetro de ordem para cristais líquidos nemáticos seja uma matriz, ao invés do escalar $Q$. Assim, o parâmetro de ordem matricial $\mathbb{Q}$ é definido por

$$
\mathbb{Q}=\left\langle\frac{1}{2}\left(3 \boldsymbol{n}_{i} \boldsymbol{n}_{i}-\boldsymbol{\delta}\right)\right\rangle
$$

e tem as componentes

$$
\mathbb{Q}_{\mu \nu}=\left\langle\frac{1}{2}\left(3 n_{i}^{\mu} n_{i}^{\nu}-\delta^{\mu \nu}\right)\right\rangle
$$

Num sistema de coordenadas cartesianas, sendo $x_{i}$ o ângulo azimutal entre o diretor local $\boldsymbol{n}_{i}$ e o eixo $z$, definido por $\boldsymbol{N}$, o parâmetro de ordem $Q$ dado por (4.1) é similar à componente $Q_{z z}$ em (4.5). Entretanto, a matriz $\mathbb{Q}$ em (4.4) é evidentemente mais geral. Em particular, essa representação nos permite descrever simetria orientacional também sobre dois eixos de simetria. Para ver isso, considere a forma diagonal ${ }^{5}$ de $\mathbb{Q}$. Uma vez que o traço de $\mathbb{Q}$ é nulo, como pode ser visto da equação (4.5), podemos parametrizar a

\footnotetext{
${ }^{5} \mathbb{Q}$ é real e simétrica, podendo, portanto, ser representada numa forma diagonal.
} 
representação diagonal de acordo com a equação

$$
\mathbb{Q}=\left(\begin{array}{ccc}
-\frac{S+\eta}{2} & 0 & 0 \\
0 & -\frac{S-\eta}{2} & 0 \\
0 & 0 & S
\end{array}\right) .
$$

Portanto, a fase é isotrópica quando $S=\eta=0$, nemática unixaxial quando $S \neq 0$ e $\eta=0$, e nemática biaxial quando $S \neq 0$ e $\eta \neq 0$. A Figura 4.3 ilustra os ordenamentos nemáticos para os casos simples de discos e cilindros que estamos interessados.

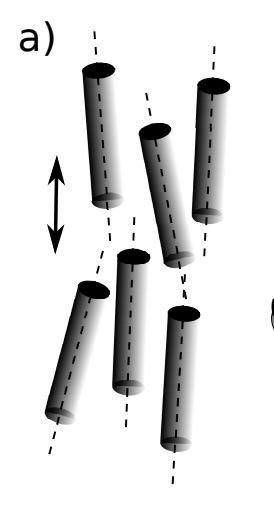

b)
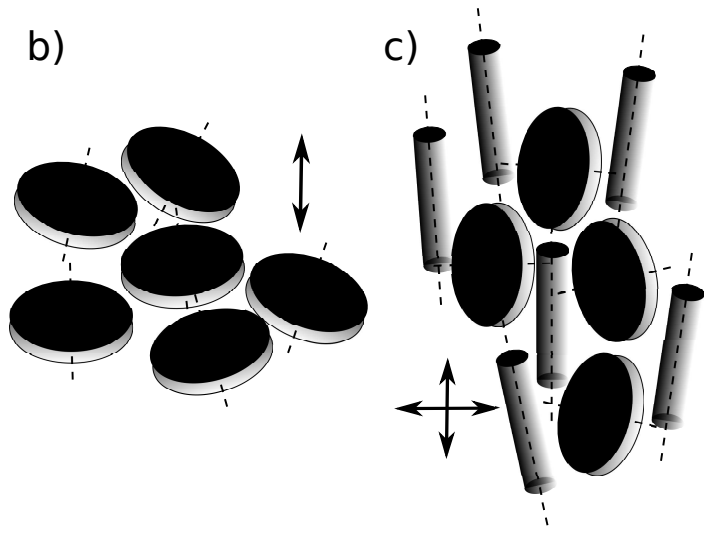

Figura 4.3: Ordenamento nemático uniaxial de cilindros (a), discos (b), e ordenamento nemático biaxial de misturas de discos e cilindros (c).

A citação de De Gennes no primeiro parágrafo deste capítulo talvez esclareça o fato de boa parte das propriedades termodinâmicas desses sistemas serem estudadas teoricamente por meio de abordagens fenomenológicas, baseadas em argumentos de simetria, como expansões do tipo Landau-De Gennes da energia livre (Gennes e Prost, 1993; Gramsbergen, Longa, e Jeu, 1986). Sob o ponto de vista microscópico, nenhuma solução exata foi obtida mesmo para os modelos estatísticos mais simples de cristais líquidos (Gennes e Prost, 1993). Abordagens microscópicas incluem interações estéricas de volume excluído para orientações contínuas (Onsager, 1949) e modelos definidos na rede (Flory, 1956; Alben, 1973), que dão origem a problemas notoriamente difíceis, além de diversos cálculos de campo médio, baseados em proposta original de Maier e Saupe (Maier e Saupe, 1958). Essa última se baseia na energia de interação entre dois mesógenos

$$
U_{i, j}=-A P_{2}\left(\cos \theta_{i j}\right)
$$


onde $\theta_{i j}$ é o ângulo entre os diretores locais indexados por $i$ e $j$. De forma similar à teoria de Weiss para o magnetismo, pode-se considerar a energia associada a um único sítio $i$ como a média

$$
U\left(\theta_{i}\right)=-\left\langle\sum_{j} A P_{2}\left(\cos \theta_{i j}\right)\right\rangle_{j} .
$$

No contexto da abordagem de Maier-Saupe pode-se pensar num modelo de Maier-Saupe (Henriques e Henriques, 1997; Carmo et al., 2010), que é uma espécie de análogo nemático do modelo de Curie e Weiss (Salinas e Wreszinski, 1993), com interações de alcance infinito. Esse modelo de campo médio pode ser analisado através de cálculos estatísticos bem controlados, embora ainda não permita uma solução exata devido à dificuldade criada pela simetria contínua dos vetores de ordenamento local. Torna-se então conveniente introduzir uma simplificação adicional, que consiste na restrição dos ordenamentos locais a um conjunto discreto de orientações, como foi proposto inicialmente por Robert Zwanzig para o modelo de Onsager (Zwanzig, 1963), dando origem ao modelo de Maier-SaupeZwanzig, ou modelo MSZ, que pode ser tratado analiticamente com bastante detalhe. Esta restrição nos graus orientacionais de liberdade não deve afetar o comportamento qualitativo do sistema contínuo, como foi verificado no caso de mesógenos com simetria uniaxial (Oliveira e Neto, 1986). Retornaremos ao modelo de Maier-Saupe-Zwanzig na seção 4.5 .

\subsection{O problema físico}

A primeira predição teórica sobre a possibilidade de um ordenamento nemático biaxial em cristais líquidos remonta ao início da década de 70, e é atribuída a um trabalho pioneiro de Freiser (Freiser, 1970). Desde então, tão grande tem sido a repercussão entre teóricos e experimentais, que a busca por materiais termotrópicos nemáticos biaxiais foi apelidada "the holy grail" dos cristais líquidos (Luckhurst, 2004). Os primeiros frutos desta busca apareceram apenas na década passada, com os trabalhos experimentais de Madsen et al. (Madsen, Dingemans, Nakata, e Samulski, 2004) e Acharya et al. (Acharya, Primak, e Kumar, 2004) com moléculas anisotrópicas em forma de banana. Em cristais líquidos 
liotrópicos, entretanto, a fase nemática biaxial foi encontrada ainda no início da década de 80, por Yu e Saupe, numa mistura ternária de laurato de potássio, decanol e água deuterada (Yu e Saupe, 1980).

Segundo os resultados de Yu e Saupe, a região ordenada de um diagrama de fases "temperatura versus concentração" apresenta uma fase nemática biaxial, localizada entre duas fases uniaxiais associadas a agregados moleculares de formas lamelar e calamítica ${ }^{6}$, limitadas por fronteiras de transição de segunda ordem (Figura 4.4). Transições entre as fases nemáticas e a fase isotrópica, por sua vez, são descontínuas, e a linha crítica contínua entre as fases uniaxiais e a fase biaxial termina aparentemente em um único ponto, conhecido em fenômenos críticos como ponto multicrítico de Landau (Yu e Saupe, 1980).

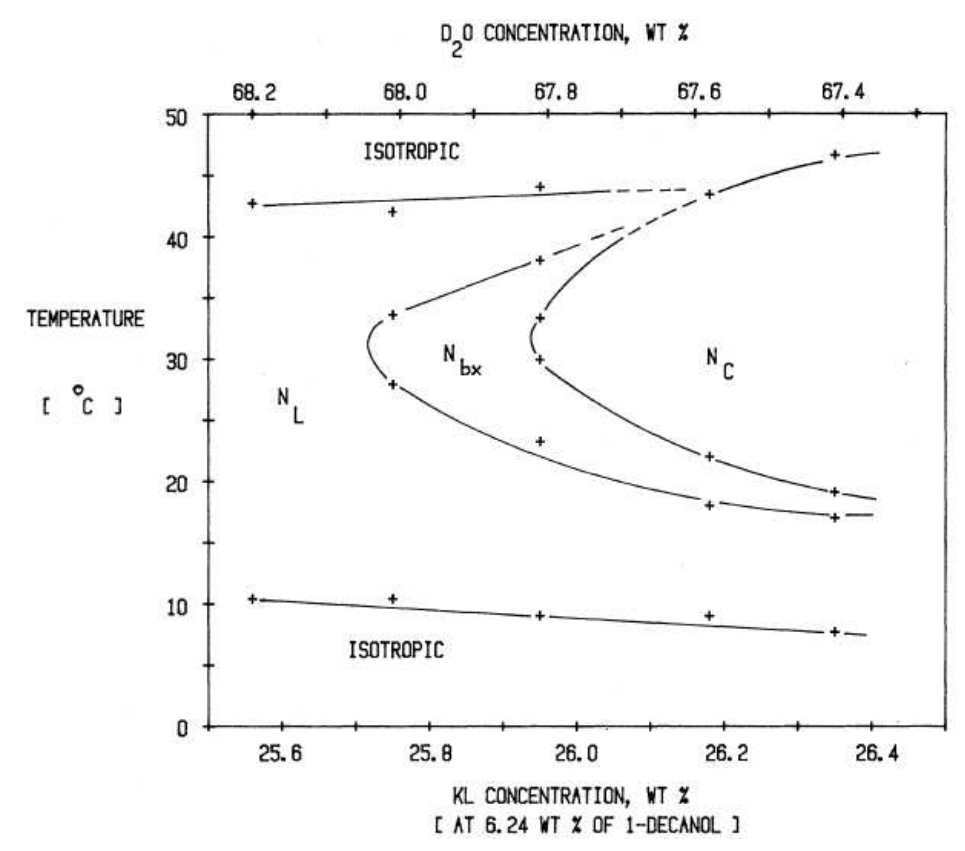

Figura 4.4: Diagrama de fases de uma mistura ternária de água deuterada, decanol e laurato de potássio. Fonte: (Yu e Saupe, 1980).

Diferentes abordagens divergem sobre a descrição microscópica desses materiais. Para misturas de discos e cilindros, modelos estatísticos na rede, introduzidos pouco após o trabalho de Freiser, indicam a presença de um ordenamento nemático biaxial (Alben, 1973;

\footnotetext{
${ }^{6}$ Há um contínuo de formas associadas aos agregados moleculares desse sistema a medida em que a concentração de decanol é variada.
} 
Chen e Deutch, 1984). Por outro lado, cálculos de campo médio baseados em interações quadrupolares, como consideradas por Maier e Saupe, resultam em uma fase nemática biaxial instável, a menos que sejam satisfeitas condições muito especiais (Palffy-Muhoray, Bruyn, e Dunmur, 1985a, 1985b; Sharma, Palffy-Muhoray, Bergesersen, e Dunmur, 1985). Simulações numéricas corroboram esses resultados, de acordo com o artigo de revisão recente de Berardi et al., que afirmam que quase todas as tentativas de simulações de misturas de discos e cilindros, para modelos que não são definidos na rede, falharam em detectar uma fase nemática biaxial (Berardi, Muccioli, Orlandi, Ricci, e Zannoni, 2008). Enfatizamos, porém, que a maioria das simulações considerava modelos baseados em interações de volume excluído.

Mais de uma década após os trabalhos de Palffy-Muhorey et al., Henriques e Henriques analisaram uma versão discretizada do modelo de Maier-Saupe, com a introdução de variáveis aleatórias de desordem temperada, representando a polidispersão de micelas com simetria axial ${ }^{7}$ (Henriques e Henriques, 1997), obtendo como resultado uma fase nemática biaxial estável no diagrama de fases (Figura 4.5a). Polimorfismo temperado, entretanto, pode não representar adequadamente a situação encontrada em sistemas fluidos, como cristais líquidos. Tendo em mente misturas de discos e cilindros, Carmo et al. analisaram um modelo inspirado no modelo definido em (Henriques e Henriques, 1997), porém com uma distribuição recozida de formas moleculares (Carmo et al., 2010). Os resultados concordam com os trabalhos de Palffy-Muhorey et al., isto é, para distribuições recozidas de variáveis de desordem a fase nemática biaxial é instável (Figura 4.5b). Fomos então motivados a considerar uma situação de desordem intermediária, que não poderia ser descrita por variáveis nem puramente temperadas, nem puramente recozidas, e a verificar que condições levariam à existência e estabilidade da fase biaxial (Carmo et al., 2010).

\footnotetext{
${ }^{7}$ Neste trabalho são considerados os casos bidisperso (mistura de discos e cilindros) e polidisperso (distribuição contínua de formas moleculares).
} 

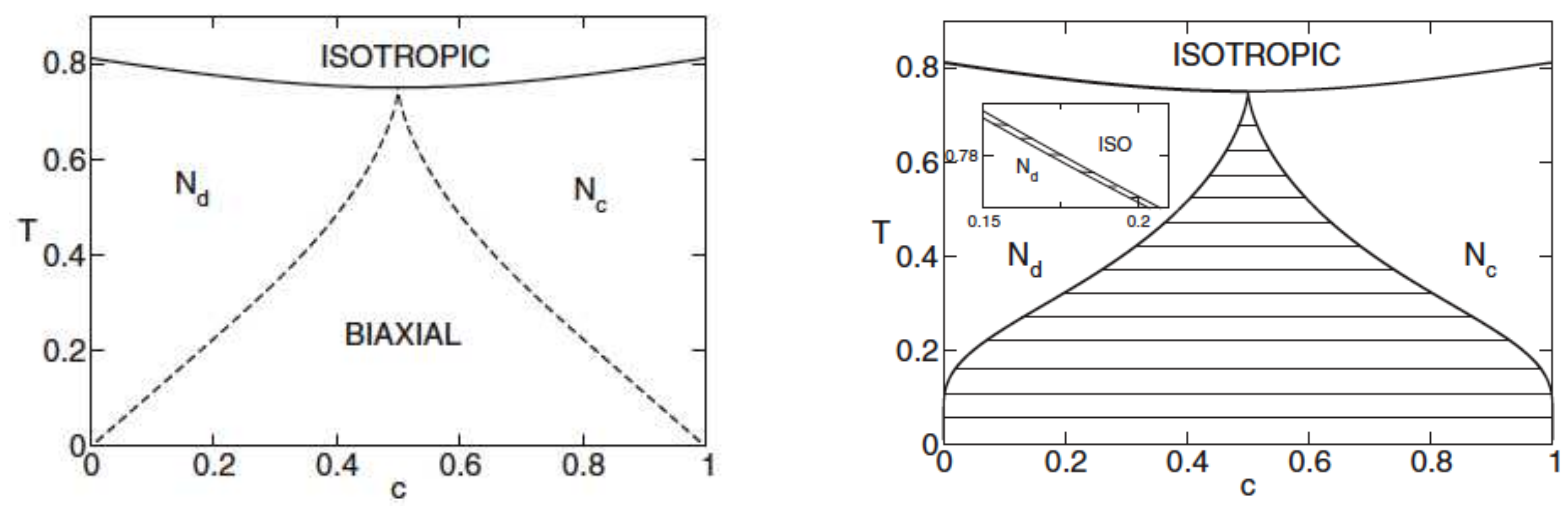

Figura 4.5: Diagrama de fases temperatura versus concentração para misturas de discos e cilindros para variáveis de desordem temperadas (à esquerda), e recozidas (à direita). ISOTROPIC, BIAXIAL, $\mathrm{N}_{\mathrm{d}}$ e $\mathrm{N}_{\mathrm{c}}$ denotam as fases isotrópica, nemática biaxial, nemática uniaxial discótica e nemática uniaxial cilíndrica, respectivamente. Fonte: (Carmo et al., 2010).

\subsection{Formulação estatística em duas temperaturas}

Com o intuito de abordar analiticamente um problema de desordem intermediária, Coolen et al. introduziram um modelo cuja dinâmica acopla variáveis de spin "rápidas" e de interação "lentas", motivados por leis sugeridas por processos neurais (Coolen, Penney, e Sherrington, 1993). Segundo esta proposta, spins e parâmetros de interação estão acoplados a banhos térmicos a temperaturas diferentes. É possível então demonstrar, partindo de argumentos heurísticos, que o sistema, no "equilíbrio" 8 , é equivalente a um sistema recozido de $n$ réplicas, onde o número de réplicas corresponde ao quociente das duas temperaturas e pode assumir qualquer valor real positivo. Os limites $n \rightarrow 0$ e $n \rightarrow 1$ correspondem às desordens temperada e recozida, respectivamente. A evolução dinâmica de um sistema de spins com desordem intermediária foi analisada também por Nestor Caticha, por meio de equações mestras na aproximação de campo médio, num modelo onde os limites temperado e recozido correspondem aos modelos de Hopfield e de Ashkin e Teller, respectivamente (Caticha, 1994).

A descrição de sistemas em termos de duas temperaturas, efetivas neste caso, tem sido empregada também no estudo de vidros estruturais, onde as tentativas de generalizar a

\footnotetext{
${ }^{8}$ Estritamente falando, pode-se falar apenas em equilíbrio local em um modelo definido em duas temperaturas.
} 
termodinâmica de forma a descrever satisfatoriamente o comportamento vítreo têm uma história longa (Kurchan, 2005; Leuzzi, 2009). Sendo materiais terminantemente fora do equilíbrio, vidros fornecem um exemplo claro de separação entre as escalas de tempo de dois grupos de variáveis dinâmicas. Temperaturas efetivas, com várias das propriedades esperadas para uma temperatura de equilíbrio, tem sido então propostas, levando à prescrição de duas temperaturas, distintas e aparentemente mensuráveis experimentalmente (Greinert, Wood, e Barlett, 2006), para descrever um vidro a baixas temperaturas (Cugliandolo, Kurchan, e Peliti, 1997).

Seguiremos uma adaptação da formulação heurística proposta nas referências (Allahverdyan, Nieuwenhuizen, e Saakian, 2000) e (Allahverdyan e Petrosyan, 2006), que apresentaremos adiante. Allahverdyan et al. partem das hipóteses de separação adiabática das escalas de tempo e da existência de duas temperaturas fixas para os diferentes componentes para desenvolver uma termodinâmica generalizada fora do equilíbrio para sistemas desordenados (Allahverdyan e Nieuwenhuizen, 2000; Allahverdyan et al., 2000). Resultados surpreendentes podem provir desta formulação. Por exemplo, numa versão de Curie e Weiss do modelo de Ising diluído, esta teoria pode levar à existência de um calor latente positivo quando o sistema passa de uma fase de maior para uma de menor temperatura, um cenário impossível no contexto de transições de fase de equilíbrio. Neste sistema, a abordagem em duas temperaturas é motivada por experimentos de espectroscopia de ressonância nuclear magnética (NMR) e de ressonância eletrônica de spin (ESR), que fornecem evidências bastante convincentes de que a temperatura do banho térmico dos spins é de fato diferente da temperatura da rede, para um intervalo de escala de tempos (Allahverdyan e Petrosyan, 2006) (Figura 4.6). Outras aplicações incluem descrições de sistemas acoplados de gases ideais, de um gás ideal que interage com um oscilador harmônico (Ritter, D'Ajello, e Figueiredo, 2004), e de ecossistemas complexos, onde a abundância de espécies e as interações entre espécies variam com o tempo, embora com escalas de tempo distintas (Poderoso e Fontanari, 2007; Poderoso, 2007).

Considere $T_{\lambda}$ a temperatura do banho térmico referente a um conjunto de variáveis aleatórias de desordem $\left\{\lambda_{i}\right\}$, e $T$ a temperatura do banho térmico dos spins ${ }^{9}$. Se admitir-

\footnotetext{
${ }^{9}$ Trataremos adiante variáveis dinâmicas relacionadas ao momento de quadrupolo, ao invés do spin, quando nos referirmos
} 


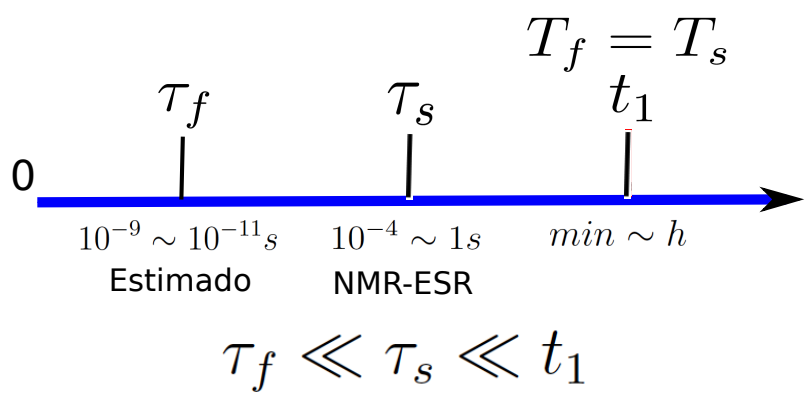

Figura 4.6: O tempo estimado para as variáveis associadas à diluição da rede alcançarem equilíbrio à temperatura $T_{f}$ é de aproximadamente $10^{-9} \sim 10^{-11} \mathrm{~s}$. De acordo com medidas de NMR e ESR, as variáveis de spins são termalizadas à temperatura $T_{s}$ entre $10^{-4} \mathrm{e} 1 \mathrm{~s}$. As duas temperaturas precisam de minutos ou mesmo horas para se igualarem. A hipótese de duas temperaturas é, portanto, razoável para um regime onde $\tau_{f} \ll \tau_{s} \ll t_{1}$.

mos que as variáveis de spin se termalizam para cada dada configuração fixa do conjunto $\{\lambda\}$, temos a seguinte distribuição de equilíbrio para o conjunto $\{\sigma\}$ :

$$
P(\sigma \mid \lambda)=\frac{\exp [-\beta \mathcal{H}(\sigma, \lambda)]}{Z_{\sigma}}
$$

onde $Z_{\sigma}=Z_{\sigma}(\lambda)=\operatorname{Tr}_{\sigma} \exp [-\beta \mathcal{H}(\sigma, \lambda)]$, com o traço tomado apenas sobre as variáveis de spin $\{\sigma\}, \beta=1 / k T$ e $\mathcal{H}$ é a hamiltoniana do sistema. Supomos agora que as variáveis de desordem não são fixas, mas evoluem com o tempo. Descrevemos a evolução temporal das variáveis $\lambda_{i}$ pelo conjunto de equações de Langevin,

$$
\Gamma \frac{\partial \lambda_{i}}{\partial t}=-z(t) \lambda_{i}-\frac{\partial \mathcal{H}}{\partial \lambda_{i}}+\eta_{i}(t), \quad 1 \leq i \leq N
$$

onde $z(t)$ é um multiplicador de Lagrange que garante a concentração de cada tipo de partícula $^{10}$ e $\Gamma$ é uma constante de amortecimento. As variáveis de ruído $\left\{\eta_{i}(t)\right\}$ são aleatórias, independentes e identicamente distribuídas com média zero e variância

$$
\left\langle\eta_{i}(t) \eta_{j}\left(t^{\prime}\right)\right\rangle=2 \Gamma T_{\lambda} \delta_{i j} \delta\left(t-t^{\prime}\right)
$$

ao modelo de Maier-Saupe. Simplificamos a linguagem apenas para a exposição da formulação estatística. É importante notar que a suposição da existência de dois banhos térmicos a temperaturas distintas não possui evidências experimentais suficientes no problema que abordaremos, como ocorre no caso do magneto de Ising diluído, de forma que temos que admitir a existência de escalas de tempos que satisfaçam essa hipótese.

${ }^{10}$ Analisaremos a seguir uma hamiltoniana onde as variáveis $\lambda_{i}$ podem representar moléculas prolatas (cilíndricas) ou oblatas (discóticas), dependendo do sinal. 
onde o fator $2 \Gamma T_{\lambda}$ provém da relação de Einstein, necessária para garantir a distribuição de equilíbrio de Gibbs. Desde que haja uma separação das escalas de tempo com duas temperaturas distintas para os diferentes componentes do sistema (Allahverdyan et al., 2000), podemos admitir que as variáveis de spin se termalizam para cada valor do conjunto $\left\{\lambda_{i}\right\}$, e que o termo $\partial \mathcal{H} / \partial \lambda_{i}$ pode ser substituído pelo seu valor médio ${ }^{11}$ em $\sigma$, que pode ser escrito na forma

$$
\left\langle\frac{\partial \mathcal{H}}{\partial \lambda_{i}}\right\rangle_{\sigma}=\frac{\partial \mathcal{H}_{\mathrm{eff}}}{\partial \lambda_{i}}
$$

onde a hamiltoniana efetiva $\mathcal{H}_{\text {eff }}$ é dada por

$$
\mathcal{H}_{\text {eff }}=\mathcal{H}_{\text {eff }}(\lambda)=-T \ln \operatorname{Tr}_{\sigma} \exp (-\beta \mathcal{H})
$$

Especifiquemos o sistema como uma mistura de duas componentes, que podem ter forma prolata (cilíndrica), com $\lambda_{i}=+1$, ou oblata (discótica), com $\lambda_{i}=-1$, tal que

$$
\sum_{i} \lambda_{i}=N_{p}-N_{o}, \quad N=N_{p}+N_{o}
$$

onde $N$ é o número total de partículas, $N_{p}\left(N_{o}\right)$ é o número de partículas prolatas (oblatas). As equações (4.10-4.13) resultam na distribuição de equilíbrio das variáveis $\lambda$,

$$
P(\lambda)=\frac{e^{\beta_{\lambda} \mu N_{p}} e^{-\beta_{\lambda} \mathcal{H}_{\mathrm{eff}}}}{\Xi\left(\beta_{\lambda}, \beta, N, \mu\right)},
$$

$\operatorname{com}^{12}$

$$
\Xi=\sum_{N_{p}=0}^{N} e^{\beta_{\lambda} \mu N_{p}} \sum_{\left\{\lambda_{i}\right\}}^{\prime} e^{-\beta_{\lambda} \mathcal{H}_{\mathrm{eff}}}
$$

onde $\mu$ é o potencial químico e $\sum^{\prime}$ denota uma soma sobre os valores de $\lambda_{i}$ com a restrição $\sum_{i} \lambda_{i}=2 N_{p}-N$, e $\beta_{\lambda}=1 / k T_{\lambda}$. Podemos manipular a equação (4.16) de forma a escrever $\Xi$ numa forma mais apropriada,

$$
\Xi=\sum_{\left\{\lambda_{i}\right\}}\left\{\operatorname{Tr}_{\sigma} \exp \left[-\beta\left(\mathcal{H}-\frac{\mu}{2}\left(\sum_{i} \lambda_{i}+N\right)\right)\right]\right\}^{\frac{\beta_{\lambda}}{\beta}},
$$

\footnotetext{
${ }^{11}$ Supomos que o tempo de relaxação das variáveis de spin seja muito menor que o das variáveis de desordem $\lambda_{i}$.

${ }^{12}$ Note que um potencial químico é suficiente para descrever uma mistura de duas componentes. O número total de partículas $N$ é fixo, de forma que, para cada $N_{1}$, não existem dois estados microscópicos com valores distintos de $N_{2}$. O potencial $\mu$ "cria" moléculas prolatas e "destrói" moléculas oblatas simultaneamente.
} 
onde a soma sobre $\{\lambda\}$ não possui mais restrições. A energia livre é obtida da equação

$$
-\beta_{\lambda} \psi=\lim _{N \rightarrow \infty} \frac{1}{N} \ln \Xi
$$

ou seja,

$$
-\beta \psi=\lim _{N \rightarrow \infty} \frac{1}{N n} \ln \sum_{\left\{\lambda_{i}\right\}} Z_{\mathrm{eff}^{n}}
$$

onde

$$
Z_{\text {eff }}=\operatorname{Tr}_{\sigma} \exp \left\{-\beta\left[\mathcal{H}-\frac{\mu}{2}\left(\sum_{i} \lambda_{i}+N\right)\right]\right\},
$$

e $n=\beta_{\lambda} / \beta$. A equação (4.19) é semelhante à expressão para a energia livre temperada de um modelo de vidros de spins segundo o método das réplicas ${ }^{13}$ (Binder e Young, 1986).

\subsection{Modelo de Maier-Saupe-Zwanzig desordenado}

Vamos considerar um modelo de Maier-Saupe desordenado para misturas de discos e cilindros (Henriques e Henriques, 1997; Carmo et al., 2010), cuja hamiltoniana é dada por

$$
\mathcal{H}=-\frac{A}{N} \sum_{i<j} \sum_{\mu, \nu} \lambda_{i} \lambda_{j} S_{i}^{\mu \nu} S_{j}^{\mu \nu}
$$

com

$$
\begin{gathered}
S_{i}^{\mu \nu}=\frac{1}{2}\left(3 n_{i}^{\mu} n_{i}^{\nu}-\delta_{\mu \nu}\right), \\
\left|\boldsymbol{n}_{i}\right|=1, \quad i=1,2, \cdots N,
\end{gathered}
$$

onde $n_{i}^{\mu}$ denota a componente $\mu$ do vetor diretor local $\boldsymbol{n}_{i}, A$ é um parâmetro de energia, e as variáveis aleatórias de desordem $\lambda_{i}$ modelam a forma dos mesógenos, tendo valor $+1(-1)$ para formas cilíndricas (discóticas). Além disso, $i$ e $j$ indexam os mesógenos e as somas sobre $\mu$ e $\nu$ percorrem as componentes $x, y$ e $z$ em coordenadas cartesianas ${ }^{14}$. Assim, de acordo com a equação (4.20),

$$
Z_{\text {eff }}=\operatorname{Tr}_{\boldsymbol{n}} \exp \left\{-\beta\left[\mathcal{H}-\frac{\mu}{2}\left(\sum_{i} \lambda_{i}+N\right)\right]\right\},
$$

\footnotetext{
${ }^{13}$ Para modelos de vidros de spin é necessário tomar-se o limite $n \rightarrow 0$.

${ }^{14} \mathrm{~A}$ hamiltoniana (4.21) é uma versão do tipo Curie-Weiss da teoria de Maier-Saupe, mencionada na seção 4.1, com a adição de variáveis aleatórias de desordem.
} 
e, portanto, para $n=\beta_{\lambda} / \beta$ inteiro $^{15}$,

$$
Z_{\mathrm{eff}}^{n}=\exp \left[\frac{\beta \mu n}{2}\left(\sum_{i} \lambda_{i}+N\right)\right] \operatorname{Tr}_{\boldsymbol{n} n} \exp \left(-\beta \sum_{\alpha=1}^{n} \mathcal{H}^{\alpha}\right)
$$

onde introduzimos o índice $\alpha$ para denotar cada réplica do sistema. O traço $\left(\operatorname{Tr}_{\boldsymbol{n} n}\right)$ agora corresponde a uma soma sobre as configurações para as $n$ réplicas. Tendo em vista a equação (4.21), obtemos, desprezando termos de ordem $N^{-1}$,

$$
Z_{\mathrm{eff}}{ }^{n}=\exp \left[\frac{\beta \mu n}{2}\left(\sum_{i} \lambda_{i}+N\right)\right] \operatorname{Tr}_{\sigma n} \prod_{\mu, \nu,(\alpha)} \exp \left(z a^{2}\right),
$$

onde $z=\beta A / 2 N$ e $a=\sum_{i} \lambda_{i} S_{i}^{\mu \nu(\alpha)}$. A soma sobre as partículas pode ser linearizada se aplicarmos as identidades gaussianas

$$
e^{z a^{2}}=\sqrt{\frac{\beta A N}{2 \pi}} \int_{-\infty}^{\infty} d Q_{\mu \nu}^{(\alpha)} \exp \left(-\frac{N \beta A}{2} Q_{\mu \nu}^{(\alpha)^{2}}+\beta A \sum_{i} \lambda_{i} S_{i}^{\mu \nu(\alpha)} Q_{\mu \nu}^{(\alpha)}\right)
$$

de forma que

$$
\begin{aligned}
Z_{\mathrm{eff}}^{n}=\exp & {\left[\frac{\beta \mu n}{2}\left(\sum_{i} \lambda_{i}+N\right)\right] \operatorname{Tr}_{\sigma n} \prod_{\mu, \nu,(\alpha)} \sqrt{\frac{\beta A N}{2 \pi}} \int_{-\infty}^{\infty} d Q_{\mu \nu}^{(\alpha)} } \\
& \times \exp \left(-\frac{N \beta A}{2} Q_{\mu \nu}^{(\alpha)^{2}}+\beta A \sum_{i} \lambda_{i} S_{i}^{\mu \nu(\alpha)} Q_{\mu \nu}^{(\alpha)}\right)
\end{aligned}
$$

A soma sobre $\{\lambda\}$ resulta, então,

$$
\begin{aligned}
\sum_{\left\{\lambda_{i}\right\}} Z_{\mathrm{eff}}^{n}= & e^{\frac{\beta \mu N n}{2}} \int d \vec{Q} \exp \left(-\frac{N \beta A}{2} \sum_{\alpha} \sum_{\mu, \nu} Q_{\mu \nu}^{(\alpha)^{2}}\right) \\
& \times \prod_{i}\left\{\sum_{S_{i}^{\mu \nu(\alpha)}} \sum_{\lambda_{i}} \exp \left[\beta \lambda_{i}\left(A \sum_{\alpha} \sum_{\mu, \nu} S_{i}^{\mu \nu(\alpha)} Q_{\mu \nu}^{(\alpha)}+\frac{\mu n}{2}\right)\right]\right\} \\
= & e^{\frac{\beta \mu N n}{2}} \int d \vec{Q} \exp \left(-\frac{N \beta A}{2} \sum_{\alpha} \sum_{\mu, \nu} Q_{\mu \nu}^{(\alpha)^{2}}+N \ln V\right),
\end{aligned}
$$

onde

$$
d \vec{Q}=\prod_{\mu, \nu,(\alpha)} \sqrt{\frac{\beta A N}{2 \pi}} d Q_{\mu \nu}^{(\alpha)}
$$

\footnotetext{
${ }^{15}$ Aplicaremos o método das réplicas sem tomar o limite $n \rightarrow 0$ ao final, mas sim uma continuação analítica para $n \in \mathbb{R}^{+}$.
} 
e $V$ é dado por

$$
\begin{aligned}
V & =\sum_{S^{\mu \nu(\alpha)}} \sum_{\lambda} \exp \left[\beta \lambda\left(A \sum_{\alpha} \sum_{\mu, \nu} S^{\mu \nu(\alpha)} Q_{\mu \nu}^{(\alpha)}+\frac{\mu n}{2}\right)\right] \\
& =\sum_{\lambda} e^{\frac{\beta \mu n \lambda}{2}} \prod_{\alpha}\left[\sum_{S^{\mu \nu(\alpha)}} \exp \left(\beta \lambda A \sum_{\mu, \nu} S^{\mu \nu(\alpha)} Q_{\mu \nu}^{(\alpha)}\right)\right] .
\end{aligned}
$$

Note que o traço agora é calculado sobre os estados de um único sítio.

Ao invés de somarmos sobre todos os estados definidos na esfera $|\boldsymbol{n}|=1$, adotaremos a proposta simplificadora de Zwanzig, segundo a qual o número de configurações possíveis do vetor diretor $\boldsymbol{n}$ é discretizado e restrito às seis direções ao longo dos três eixos cartesianos, ou seja

$$
\boldsymbol{n}_{i} \in\{( \pm 1,0,0),(0, \pm 1,0),(0,0, \pm 1)\}
$$

Assim,

$$
\sum_{S^{\mu \nu(\alpha)}} \exp \left(\beta \lambda A \sum_{\mu, \nu} S^{\mu \nu(\alpha)} Q_{\mu \nu}^{(\alpha)}\right)=\exp \left(-\frac{\beta \lambda A}{2} \sum_{\mu} Q_{\mu \mu}^{(\alpha)}+\ln 2\right)\left(\sum_{\mu} e^{\frac{3 \beta \lambda A}{2} Q_{\mu \mu}^{(\alpha)}}\right)
$$

Portanto,

$$
\begin{array}{r}
V=2^{n} \sum_{\lambda}\left[\prod_{\alpha}\left(\sum_{\mu} e^{\frac{3 \beta \lambda A}{2} Q_{\mu \mu}^{(\alpha)}}\right)\right] \exp \left[\frac{\beta \lambda}{2}\left(\mu n-A \sum_{\mu,(\alpha)} Q_{\mu \mu}^{(\alpha)}\right)\right] \\
=2^{n}\left\{\exp \left[\frac{\beta}{2}\left(\mu n-A \sum_{\mu,(\alpha)} Q_{\mu \mu}^{(\alpha)}\right)\right] \prod_{\alpha}\left(\sum_{\mu} e^{\frac{3 \beta A}{2} Q_{\mu \mu}^{(\alpha)}}\right)\right. \\
\left.+\exp \left[-\frac{\beta}{2}\left(\mu n-A \sum_{\mu,(\alpha)} Q_{\mu \mu}^{(\alpha)}\right)\right] \prod_{\alpha}\left(\sum_{\mu} e^{-\frac{3 \beta A}{2} Q_{\mu \mu}^{(\alpha)}}\right)\right\} .
\end{array}
$$

Apenas variáveis de um índice da energia replicada estão presentes na expressão da média de $Z_{\text {eff }}$. Essa é uma consequência da definição da hamiltoniana, com as variáveis de desordem $\lambda_{i}$ associadas a cada sítio separadamente. Desta forma, para a energia livre convergir, devemos supor simetria entre réplicas, isto é,

$$
Q_{\mu \nu}^{(\alpha)}=Q_{\mu \nu}^{(\beta)}=Q_{\mu \nu}, \quad \forall \alpha, \beta, \mu, \nu
$$

Além disto, no limite termodinâmico, $Q_{\mu \nu}=0$ para $\mu \neq \nu$, tendo em vista que $V$ não 
depende de $Q_{\mu \nu}$ neste caso $^{16}$. Logo,

$$
\sum_{\{\lambda\}} Z_{\mathrm{eff}^{n}}^{n}=e^{N n\left(\frac{\beta \mu}{2}+\ln 2\right)} \int d \vec{Q} e^{-N n g}
$$

com

$$
\begin{aligned}
g & =\frac{\beta A}{2} \sum_{\mu} Q_{\mu \mu}^{2}-\frac{1}{n} \ln \left[\left(e^{\frac{\beta}{2}\left(\mu-A \sum_{\mu} Q_{\mu \mu}\right)} \sum_{\mu} e^{\frac{3}{2} \beta A Q_{\mu \mu}}\right)^{n}\right. \\
& \left.+\left(e^{-\frac{\beta}{2}\left(\mu-A \sum_{\mu} Q_{\mu \mu}\right)} \sum_{\mu} e^{-\frac{3}{2} \beta A Q_{\mu \mu}}\right)^{n}\right]
\end{aligned}
$$

No limite $N \rightarrow \infty$, aplicamos o método de Laplace para obter uma expressão assintótica para a energia livre,

$$
\begin{aligned}
-\beta \psi & =\frac{\beta \mu}{2}+\ln 2-\frac{\beta A}{2} \sum_{\mu} Q_{\mu \mu}^{2}+\frac{1}{n} \ln \left[\left(e^{\frac{\beta}{2}\left(\mu-A \sum_{\mu} Q_{\mu \mu}\right)} \sum_{\mu} e^{\frac{3}{2} \beta A Q_{\mu \mu}}\right)^{n}\right. \\
& \left.+\left(e^{-\frac{\beta}{2}\left(\mu-A \sum_{\mu} Q_{\mu \mu}\right)} \sum_{\mu} e^{-\frac{3}{2} \beta A Q_{\mu \mu}}\right)^{n}\right]
\end{aligned}
$$

onde $\left\{Q_{\mu \mu}\right\}$ são obtidos das condições de ponto estacionário,

$$
\frac{\partial \psi}{\partial Q_{\mu \mu}}=0
$$

A matriz $\mathbb{Q}$ tem traço nulo, como se observa a partir do cálculo de $\sum_{\mu} \partial \psi / \partial Q_{\mu \mu}$.

\subsection{Teoria de Landau-De Gennes}

Nesta seção, analisaremos a estabilidade da fase nemática biaxial tendo como base a teoria fenomenológica de Landau-De Gennes, como exposta em (Gramsbergen et al., 1986). Definimos as variáveis adimensionais $\tilde{\psi}, \tilde{\mu}$ e a pelas equações

$$
\tilde{\psi}=\beta \psi, \quad \tilde{\mu}=\frac{\beta \mu}{2}, \quad a=\frac{\beta A}{2} .
$$

A energia livre fica dada então por

$$
-\tilde{\psi}=\tilde{\mu}+\ln 2-a \sum_{\mu} Q_{\mu \mu}^{2}+\frac{1}{n} \ln \left[\left(e^{\tilde{\mu}} \sum_{\mu} e^{3 a Q_{\mu \mu}}\right)^{n}+\left(e^{-\tilde{\mu}} \sum_{\mu} e^{-3 a Q_{\mu \mu}}\right)^{n}\right],
$$

e pode ser escrita como uma série de potências dos invariantes $I_{2}$ e $I_{3}$, definidos por

$$
I_{2}=\operatorname{Tr} \mathbb{Q}^{2}, \quad I_{3}=\operatorname{Tr} \mathbb{Q}^{3} .
$$

\footnotetext{
${ }^{16}$ Note que as condições de ponto estacionário resultam $\beta A Q_{\mu \nu}=0$, para todo $\mu \neq \nu$.
} 
Para este fim, usaremos o fato de que $\operatorname{Tr} \mathbb{Q}^{n}$, para $n>3$ e $\mathbb{Q}$ qualquer matriz $3 \times 3$ real e simétrica, pode ser escrito como um polinômio formado pelos invariantes $I_{1}, I_{2}$ e $I_{3}$ (Gramsbergen et al., 1986). Em geral, $\operatorname{Tr} \mathbb{Q}^{n}$ depende apenas de fatores do tipo $I_{1}^{N_{1}} I_{2}{ }^{N_{2}} I_{3}{ }^{N_{3}}$, com $N_{1}+2 N_{2}+3 N_{3}=n, N_{i} \in \mathbb{N}$. Como estamos interessados apenas nos termos da energia livre proporcionais a $I_{2}, I_{3}, I_{2}{ }^{2}$ e $I_{3}{ }^{2}$ para estudar a estabilidade da fase nemática biaxial ${ }^{17}$, podemos desprezar qualquer contribuição de $\operatorname{Tr} \mathbb{Q}^{n}$ para $n>6$. Além disto, nenhuma contribuição relevante provém do termo de ordem cinco $^{18}$, de forma que precisamos expressar apenas $\operatorname{Tr} \mathbb{Q}^{4}$ e $\operatorname{Tr} \mathbb{Q}^{6}$ como polinômios de $I_{2}$ e $I_{3}$.

Dado que $I_{1}=0$, temos $^{19}$

$$
\operatorname{Tr} \mathbb{Q}^{6}=\frac{1}{3} I_{3}^{2}+\frac{1}{4} I_{2}^{3}
$$

Para calcular o $\operatorname{Tr} \mathbb{Q}^{4}$, expressemos a matriz $\mathbb{Q}$ na forma

$$
\mathbb{Q}=\left(\begin{array}{lll}
a & 0 & 0 \\
0 & b & 0 \\
0 & 0 & c
\end{array}\right)
$$

Portanto,

$$
I_{1}^{4}=P\left(a^{4}\right)+4 P\left(a^{3} b\right)+6 P\left(a^{2} b^{2}\right)+12 P\left(a^{2} b c\right),
$$

onde $P$ denota uma soma sobre as permutações dos elementos $a, b$ e $c$ em produtos $a^{N_{a}} b^{N_{b}} c^{N_{c}}, \operatorname{com} N_{a}, N_{b}, N_{c} \in \mathbb{N}^{20}$. De forma análoga, obtemos

$$
\begin{aligned}
& I_{1}^{2} I_{2}=P\left(a^{4}\right)+2 P\left(a^{3} b\right)+2 P\left(a^{2} b^{2}\right)+2 P\left(a^{2} b c\right), \\
& I_{1} I_{3}=P\left(a^{4}\right)+P\left(a^{3} b\right), \\
& I_{2}{ }^{2}=P\left(a^{4}\right)+2 P\left(a^{2} b^{2}\right) .
\end{aligned}
$$

\footnotetext{
${ }^{17}$ Estes termos, em particular o termo proporcional a $I_{3}{ }^{2}$, são os únicos relevantes para a análise da existência e estabilidade da fase nemática biaxial (Gramsbergen et al., 1986).

${ }^{18}$ Os únicos inteiros não negativos $N_{1}, N_{2}$ e $N_{3}$ que satisfazem a relação $N_{1}+2 N_{2}+3 N_{3}=5$, dão origem aos termos $I_{1}{ }^{5}, I_{1}{ }^{3} I_{2}{ }^{2}, I_{1}{ }^{2} I_{3}, I_{1} I_{2}{ }^{2}$ e $I_{2} I_{3}$. Nenhum destes termos é relevante na análise da fase biaxial.

${ }^{19}$ Veja o Apêndice A de (Gramsbergen et al., 1986).

${ }^{20}$ Por exemplo, $P\left(a^{3} b\right)=a^{3} b+a^{3} c+b^{3} a+b^{3} c+c^{3} a+c^{3} b$.
} 
As equações (4.45-4.46) podem ser reescritas na forma matricial

$$
\left(\begin{array}{c}
I_{1}{ }^{4} \\
I_{1}{ }^{2} I_{2} \\
I_{1} I_{3} \\
I_{2}{ }^{2}
\end{array}\right)=\left(\begin{array}{cccc}
1 & 4 & 6 & 12 \\
1 & 2 & 2 & 2 \\
1 & 1 & 0 & 0 \\
1 & 0 & 2 & 0
\end{array}\right)\left(\begin{array}{c}
P\left(a^{4}\right) \\
P\left(a^{3} b\right) \\
P\left(a^{2} b^{2}\right) \\
P\left(a^{2} b c\right)
\end{array}\right)
$$

de forma que, invertendo-se (4.47),

$$
P\left(a^{4}\right)=\frac{1}{6}\left(I_{1}^{4}-6 I_{1}^{2} I_{2}+8 I_{1} I_{3}+3 I_{2}^{2}\right)
$$

ou ainda, uma vez que $I_{1}=0$,

$$
\operatorname{Tr} \mathbb{Q}^{4}=\frac{1}{2} I_{2}{ }^{2}
$$

A energia livre pode ser expressa então na forma

$$
\begin{aligned}
-\tilde{\psi}=\tilde{\mu} & +\ln 6-a I_{2}+\frac{1}{n} \ln \left[e^{\tilde{\mu} n}\left(1+\frac{3}{2} a^{2} I_{2}+\frac{3}{2} a^{3} I_{3}+\frac{9}{16} a^{4} I_{2}{ }^{2}+\frac{9}{80} a^{6} I_{3}{ }^{2}+\cdots\right)^{n}\right. \\
& \left.+e^{-\tilde{\mu} n}\left(1+\frac{3}{2} a^{2} I_{2}-\frac{3}{2} a^{3} I_{3}+\frac{9}{16} a^{4} I_{2}{ }^{2}+\frac{9}{80} a^{6} I_{3}{ }^{2}+\cdots\right)^{n}\right]
\end{aligned}
$$

onde escrevemos apenas os termos relevantes da teoria de Landau-De Gennes (termos cruzados e termos de ordem maior que $I_{2}{ }^{2}$ e $I_{3}{ }^{2}$ podem ser desconsiderados (Gramsbergen et al., 1986; Allender e Longa, 2008)). A expansão dos binômios resulta

$$
\begin{aligned}
-\tilde{\psi}=\tilde{\mu} & +\ln 6+\frac{1}{n} \ln [2 \cosh (\tilde{\mu} n)]-a I_{2}+\frac{1}{n} \ln \left[1+n \frac{3}{2} a^{2} I_{2}+n \tanh (\tilde{\mu n}) \frac{3}{2} a^{3} I_{3}\right. \\
& \left.+n\left(n-\frac{1}{2}\right) \frac{9}{8} a^{4} I_{2}{ }^{2}+n\left(n-\frac{9}{10}\right) \frac{9}{8} a^{6} I_{3}{ }^{2}+\cdots\right] .
\end{aligned}
$$

Logo,

$$
\begin{aligned}
-\tilde{\psi}=\tilde{\mu} & +\ln 6+\frac{1}{n} \ln [2 \cosh (\tilde{\mu} n)]-\left(1-\frac{3}{2} a\right) a I_{2}+\tanh (\tilde{\mu} n) \frac{3}{2} a^{3} I_{3} \\
- & \frac{9}{16} a^{4} I_{2}{ }^{2}-\left(\frac{9}{10}-n \operatorname{sech}^{2}(\tilde{\mu} n)\right) \frac{9}{8} a^{6} I_{3}{ }^{2}+\cdots
\end{aligned}
$$

Nas unidades originais, podemos escrever

$$
\frac{\psi}{A}=\frac{\psi_{0}}{A}+A^{\prime} \frac{1}{2} I_{2}+B \frac{1}{3} I_{3}+C \frac{1}{2} I_{2}^{2}+E^{\prime} I_{3}^{2}+\cdots
$$


onde

$$
\begin{aligned}
& \psi_{0}=-\frac{\mu}{2}-T\left\{\ln 6+\frac{1}{n} \ln \left[2 \cosh \left(\frac{\beta \mu n}{2}\right)\right]\right\}, \\
& A^{\prime}=1-\frac{3}{4} \beta A, \quad B=-\frac{9}{16} \beta^{2} A^{2} \tanh \left(\frac{\beta \mu n}{2}\right), \\
& C=\frac{9}{128} \beta^{3} A^{3}, \quad E^{\prime}=\frac{9}{512} \beta^{5} A^{5}\left[\frac{9}{10}-n \operatorname{sech}^{2}\left(\frac{\beta \mu n}{2}\right)\right] .
\end{aligned}
$$

Note que $C$ é sempre positivo. Se $E^{\prime}=0$, existem duas fases nemáticas uniaxiais estáveis $^{21}$ (nemática prolata para $B<0$, e oblata para $B>0$, separadas por uma linha de transição de primeira ordem) para temperaturas tais que

$$
A^{\prime}<\frac{B^{2}}{27 C}
$$

Para $E^{\prime}>0$, existe uma fase nemática biaxial estável (com energia livre menor que as das fases nemáticas uniaxiais). Esta condição é expressa da seguinte forma com relação aos parâmetros microscópicos do modelo:

$$
n<\frac{9}{10} \cosh ^{2}\left(\frac{\beta \mu n}{2}\right) .
$$

Para $\mu=0$, por exemplo, isso significa que um pequeno afastamento do caso de completa termalização, correspondente a $n=1$, restaura a estabilidade da fase nemática biaxial. Neste caso, as linhas de transição entre as fases nemáticas uniaxiais e isotrópica são obtidas das equações

$$
A^{\prime}=\frac{3}{4} C x^{2}+\frac{9}{4} E^{\prime} x^{4}, \quad B=\frac{9}{2} C x+18 E^{\prime} x^{3},
$$

e as linhas de transição entre as fases nemática biaxial e uniaxial, das equações

$$
A^{\prime}=-\frac{3}{2} C x^{2}, \quad B=-\frac{9}{2} E^{\prime} x^{3} .
$$

Não existe fase biaxial estável para $E^{\prime}<0$.

\subsection{Considerações finais}

Estudamos uma versão discretizada do modelo de Maier-Saupe desordenado num formalismo de recozimento parcial, com dois banhos térmicos a temperaturas distintas para

\footnotetext{
${ }^{21} \mathrm{~A}$ fase isotrópica permanece estável até $A^{\prime}=0$. Além disto, existe uma fase nemática uniaxial localmente estável para $B^{2} / 27 C<A^{\prime}<B^{2} / 24 C$.
} 
os momentos de quadrupolo (variáveis dinâmicas), e para as variáveis associadas ao polimorfismo molecular (variáveis de desordem). Segundo este formalismo, baseado na hipótese de separação das escalas de tempo dos dois conjuntos de variáveis, a energia livre é obtida por meio de uma média recozida de $n$ réplicas do sistema original, onde o parâmetro $n$, correspondente ao quociente das temperaturas associadas aos dois banhos térmicos, pode assumir qualquer valor real positivo. Enquanto para desordens temperadas a fase nemática biaxial é estável, para desordens recozidas existe uma linha de transição de primeira ordem separando duas fases nemáticas uniaxiais em um diagrama em termos da temperatura e do potencial químico. Por meio de uma expansão de Landau-De Gennes, verificamos que um pequeno afastamento da situação "completamente termalizada", $n=1$, é suficiente para estabilizar a fase nemática biaxial deste sistema.

Existem vários pontos em que nossa abordagem é imprecisa, dos quais destaco três. O primeiro diz respeito à aproximação de Zwanzig. Para mesógenos com simetria uniaxial, essa restrição nos graus orientacionais de liberdade parece não afetar o comportamento qualitativo do sistema contínuo (Oliveira e Neto, 1986). Resultados independentes e ainda não publicados de Eduardo Henriques e Masayuki Hase apontam na mesma direção no caso onde existe possibilidade de ordenamento biaxial. A segunda crítica está relacionada à quantidade de parâmetros de energia. Numa situação mais geral, dever-se-ia considerar ao menos três parâmetros da energia de interação entre dois tipos de partículas, $E_{A A}, E_{B B}$ e $E_{A B}$. Consideramos um regime, caracterizado por relações entre esses três parâmetros, onde existe possibilidade de ordenamento biaxial, restringindo desta forma a análise ao efeito do tipo de desordem apenas, que é o nosso objetivo principal. A terceira objeção é mais drástica, relacionada com a mensurabilidade das duas temperaturas. Embora a hipótese de separação das escalas de tempos e de temperaturas distintas ainda não possua nenhuma evidência experimental, consideramos relevantes as informações extraídas de nossa abordagem, merecendo ainda investigações futuras.

Usamos um modelo bastante simples de cristais líquidos para analisar a influência do tipo de desordem, temperada, recozida ou parcialmente recozida, na estabilidade da fase nemática biaxial. Embora este modelo não represente precisamente o cenário microscópico 
observado em cristais líquidos liotrópicos, acreditamos que as conclusões extraídas de nossa análise possam fornecer informações relevantes a futuros protocolos experimentais. Lembramos porém que, até onde os autores tem conhecimento, não há relatos na literatura sobre a observação de uma fase nemática biaxial para misturas de moléculas em formas de disco e de cilindro. No entanto, como um primeiro passo nesta direção, trabalhos recentes relatam a observação de completa miscibilidade de mesógenos de formas prolata e oblata (Kouwer e Mehl, 2003; Date e Bruce, 2003), inclusive na fase nemática (Apreutesei e Mehl, 2006). 



\section{Capítulo 5}

\section{Transições de fases em elastômeros}

\section{nemáticos}

Ainda em meados da década de setenta, Pierre-Gilles de Gennes propôs uma análise fenomenológica das propriedades termodinâmicas de um amálgama de mesógenos nemáticos a redes de polímeros (Gennes, 1975). De Gennes foi pioneiro ao perceber que redes poliméricas seriam um ponto de partida promissor na busca por um material que acoplasse ordenamento orientacional a mudanças de forma. Do ponto de vista experimental, embora existam relatos anteriores sobre os então batizados elastômeros nemáticos num estado de polidomínios, a síntese desses materiais teve seu divisor de águas com o trabalho de um grupo alemão da Universidade de Friburgo, liderado por Heino Finkelmann, que prescreve de forma robusta a produção de elastômeros nemáticos no estado monodomínio (Küpfer e Finkelmann, 1991). Desde então um enorme volume de trabalhos, teóricos e experimentais, tem sido acumulado sobre esses novos sistemas da física da matéria mole (Warner e Terentjev, 2003).

Propomos neste capítulo uma abordagem estatística elementar, talvez o modelo molecular mais simples para um elastômero nemático, capaz de dar conta de propriedades críticas de forma abrangente, e descrever o efeito de campos aleatórios. Nosso modelo combina uma versão discretizada da teoria de Maier-Saupe para cristais líquidos e uma extensão da teoria neoclássica da elasticidade de Warner-Terentjev, apropriada para sistemas de rede. Por meio de cálculos bem conhecidos, é possível obter uma curva de 
coexistência "tensão versus deformação", análoga ao diagrama $P-V$ de um fluido simples, em que um parâmetro de energia de desordem faz o papel de temperatura. Abaixo de uma tensão crítica, as linhas de coexistência se assemelham ao plateau observado experimentalmente na curva tensão versus deformação, e podem ser interpretadas como assinaturas da transição de polidomínios para monodomínio. Mostramos ainda que a presença de desordem temperada pode suavizar a transição entre as fases nemática e isotrópica, desde que as amostras tenham sido formadas no estado nemático (Liarte et al., 2011). Na seção 5.1 fazemos uma breve revisão sobre elastômeros e elastômeros nemáticos. Apresentamos as motivações na seção 5.2 e definimos o modelo de rede na seção 5.3. Calculamos a energia livre na seção 5.4. Na seção 5.5 descrevemos os resultados termodinâmicos. Por fim, na seção 5.6, discutimos alguns resultados do nosso trabalho.

\subsection{Elastômeros e elastômeros nemáticos}

Numa de suas famosas aulas, Richard Feynman explora as propriedades térmicas da borracha para ilustrar a primeira lei da termodinâmica, segundo sua maneira própria de apresentar princípios físicos conhecidos sob um novo ângulo ${ }^{1}$. A ideia de Feynman consiste em usar o fato de uma liga de borracha, que doravante chamaremos elastômero, ser contraída com a aproximação da chama de uma vela (Figura 5.1a). Tal contração poderia ser usada na fabricação de um motor térmico (Figura 5.1b). Neste exemplo o interesse de Feynman está no reconhecimento do calor como forma de energia, principal conteúdo da primeira lei da termodinâmica. Fato curioso e não intuitivo é que o aumento da temperatura leve a uma contração da borracha em questão, ao contrário do que se esperaria para a maioria dos sólidos ${ }^{2}$. Esse tipo de comportamento térmico será herdado de forma acentuada pelos elastômeros nemáticos.

Elastômeros são sólidos elásticos constituídos por redes de polímeros com conexões cruzadas $^{3}$. Apresentamos na Figura 5.2 uma ilustração bidimensional de uma dessas redes.

\footnotetext{
${ }^{1}$ Veja o Capítulo 44 de (Feynman, Leighton, e Sands, 1963).

${ }^{2}$ Igualmente curioso é o fato da água, numa de suas mais conhecidas anomalias, ser expandida quando resfriada a temperaturas de $\sim 4^{\circ} \mathrm{C}$

${ }^{3}$ Crosslinked polymer networks.
} 
a)

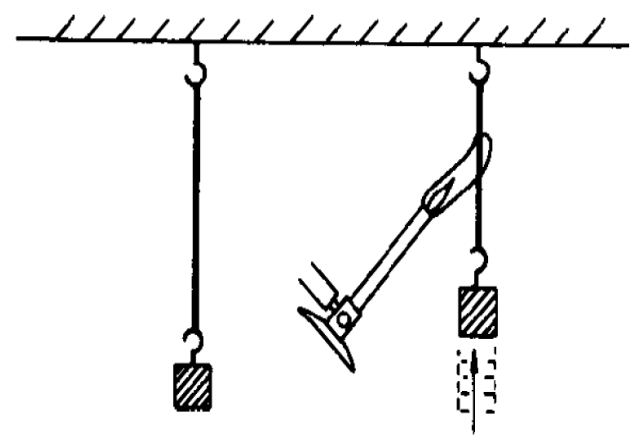

b)

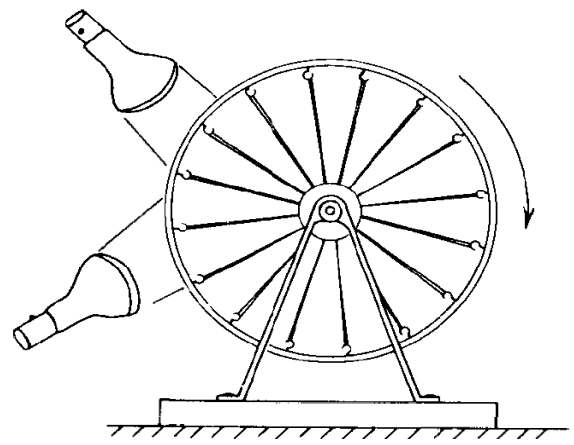

Figura 5.1: a) A aproximação da chama de uma vela a uma liga de borracha provoca uma contração na liga. b) Esboço de um possível motor térmico, alimentado por um desequilíbrio de massas gerado pelas contrações da borracha iluminada num único lado. Fonte: (Feynman, 1963).

Segmentos de reta representam monômeros de uma cadeia polimérica e elipses representam os pontos de junção entre as cadeias ${ }^{4}$. No lado direito apresentamos uma única cadeia de tamanho $\boldsymbol{R}$ entre dois pontos de junção. Essa ilustração é imprecisa em vários aspectos: 1) redes reais são tridimensionais; 2) os tamanhos dos monômeros deveriam ser aproximadamente regulares; 3) a ilustração não contempla uma densidade média de conexões cruzadas bem como tamanhos típicos entre dois pontos de junção. Não obstante, ao desprezarmos qualquer tipo de interação entre as cadeias, tal figura pode ilustrar a origem do comportamento elástico desses sistemas, viz, as formas e deformações desses materiais estão associadas a um número ótimo de configurações das cadeias poliméricas entre os pontos de junção.

Vários modelos, em geral versões de um passeio aleatório, dão conta de várias das propriedades térmicas e mecânicas de elastômeros (Flory, 1953; Kubo, 1988; Dorlas, 1999). Essas propriedades em geral estão associadas a uma contribuição predominantemente entrópica na energia livre. A origem entrópica, em conjunto com uma espécie de aproximação harmônica, rendeu a esses materiais a nomenclatura molas entrópicas. Mais do que isso, o fato de a energia livre ser independente da energia interna, ou seja, de haver completa degenerescência, fornece uma boa explicação ao fato da borracha ser contraída

\footnotetext{
${ }^{4}$ Usamos pontos de junção com um sentido geométrico, que contrasta com conexões cruzadas, de sentido físico. Ambos são escritos como crosslinks em inglês.
} 


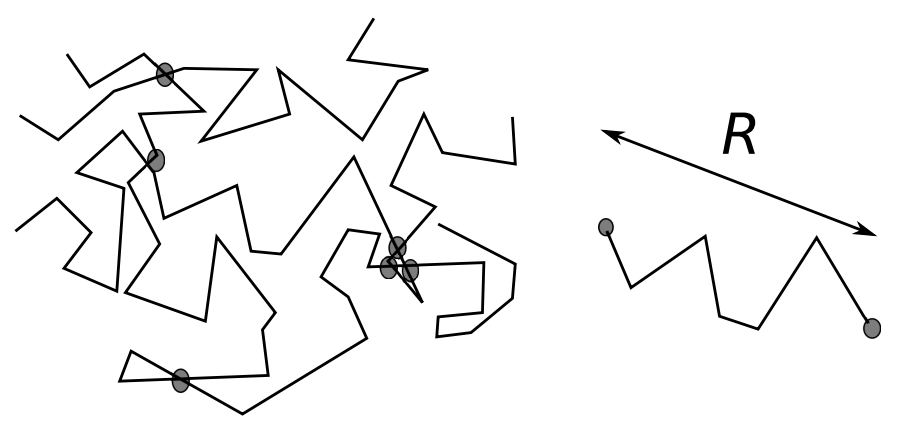

Figura 5.2: Representação de uma rede de polímeros com conexões cruzadas (à esquerda). No lado direito ilustramos uma única cadeia polimérica entre dois pontos de junção.

quando aquecida. Enquanto estudos de redes mostram que a energia interna contribui com menos de $5 \%$ para a energia livre ${ }^{5}$ em um amplo intervalo de temperaturas, esperase que com a diminuição da temperatura a contribuição da energia se torne cada vez mais relevante, culminando com uma borracha rígida. Nos cálculos adiante, admitiremos uma contribuição elástica puramente entrópica, num intervalo de temperaturas em que a borracha é, de fato, elástica.

Já discutimos algumas propriedades de cristais líquidos nemáticos no Capítulo 4. Em geral, vimos que esses materiais são constituídos por moléculas com alto grau de anisotropia de forma, que chamamos mesógenos. Esses mesógenos, quando anexados às cadeias poliméricas de elastômeros (Figura 5.3), dão origem a um novo tipo de material da física da matéria mole, que combina propriedades orientacionais de nemáticos usuais às propriedades mecânicas da borracha, os elastômeros nemáticos. Tal acoplamento gera uma estrutura capaz de grandes deformações durante a transição entre as fases isotrópica e nemática (Figura 5.4b). De forma análoga, um aumento da tensão aplicada na amostra pode induzir uma transição nemática (Figura 5.4a). Esse comportamento sugere aplicações diversas, em áreas como óptica (lentes bifocais) ou na produção de dispositivos termo-mecânicos, com interesse na fabricação de músculos artificiais (Warner e Terentjev, 2003).

\footnotetext{
${ }^{5}$ Veja o Capítulo 3 de (Warner e Terentjev, 2003).
} 


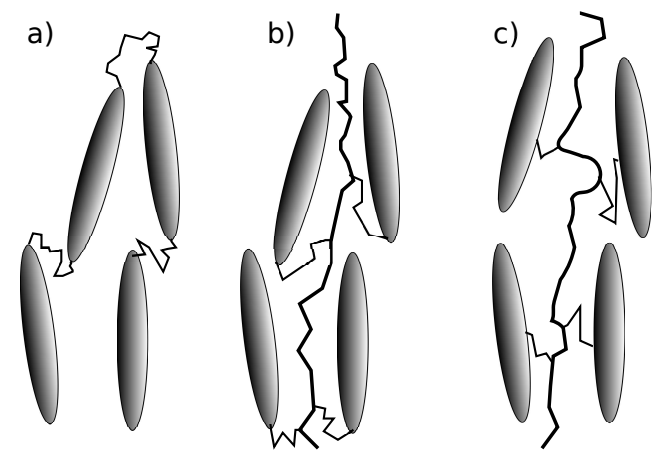

Figura 5.3: Polímeros nemáticos com mesógenos anexados à cadeia principal (a); à cadeia lateral (b); à cadeia lateral em forma de $\mathrm{T}$ (c).

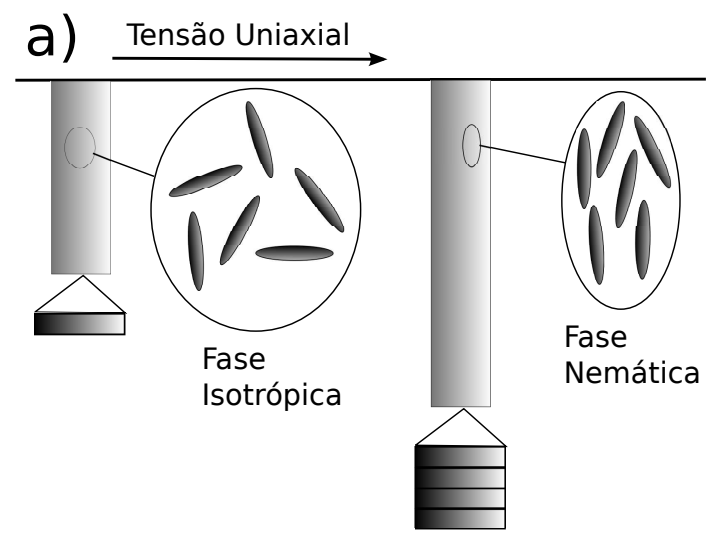

b) $\stackrel{\text { Resfriamento }}{\longrightarrow}$

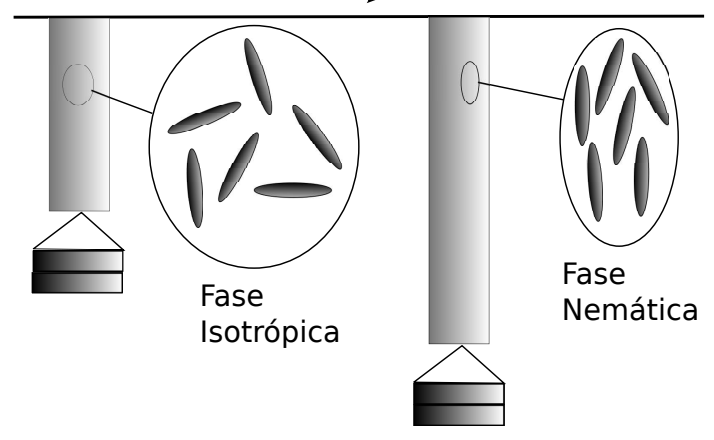

Figura 5.4: Comportamento de elastômeros nemáticos sujeitos a um aumento da tensão a temperatura fixa (a), e a uma diminuição da temperatura a tensão fixa (b).

\subsection{O problema físico}

Entre os problemas de maior interesse teórico dos elastômeros nemáticos (ENs) está a caracterização correta de suas transições de fases. Em contraste com cristais líquidos convencionais, ENs apresentam uma transição peculiar entre as fases nemática e isotrópica. Em nemáticos usuais, argumentos de simetria provenientes da teoria de Landau-De Gennes apontam para a existência de uma transição de primeira ordem, com um salto do parâmetro de ordem nemático na temperatura de transição $T_{\mathrm{NI}}$. Por sua vez, ao invés de uma descontinuidade, ENs apresentam uma variação contínua, embora rápida, do parâmetro de ordem na temperatura de transição (Clarke, Tajbakhsh, Terentjev, e War- 
ner, 2001) (Figura 5.5). O mecanismo microscópico por trás deste comportamento não trivial foi discutido por vários autores (Uchida, 2000; Selinger, Jeon, e Ratna, 2002; Selinger e Ratna, 2004; Petridis e Terentjev, 2006; Jayasri, Satyavathi, Sastry, e Murthy, 2009). Em particular, teorias contínuas macroscópicas (Petridis e Terentjev, 2006), e simulações numéricas de modelos microscópicos (Selinger e Ratna, 2004) indicam que a interação com campos aleatórios temperados pode suavizar a transição de primeira ordem nesses sistemas. Em contraste com outros sistemas, campos aleatórios possuem uma interpretação clara e bem definida em ENs, sendo supostamente originados de tensões internas produzidas pelas conexões cruzadas da rede de polímeros ${ }^{6}$. Mais recentemente, variações de campos aleatórios foram sugeridas por Lu e colaboradores para descrever heterogeneidade da rede em materiais com conexões cruzadas aleatórias (Lu, Ye, Xing, e Goldbart, 2011).

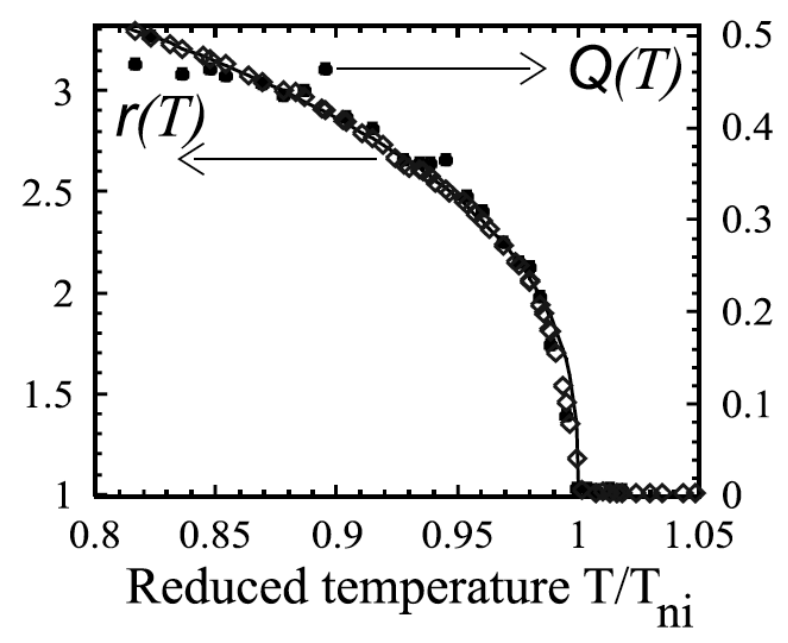

Figura 5.5: Parâmetro de ordem nemático $Q$ e anisotropia efetiva $r$ como função da temperatura reduzida $T / T_{\mathrm{NI}}$. Fonte: (Clarke et al., 2001).

Campos aleatórios também são relevantes para descrever a transição entre estados de polidomínios para monodomínio orientacional (Fridrikh e Terentjev, 1999). De fato, para $T<T_{\mathrm{NI}}$, ENs podem apresentar uma estrutura estável conhecida como Schlieren texture caracterizada por conjuntos de domínios com orientações aleatórias e congeladas

\footnotetext{
${ }^{6}$ Veja as referências (Fridrikh e Terentjev, 1997; Yu, Taylor, e Terentjev, 1998) e a Figura 5.8 para uma ilustração desses efeitos.
} 
dos mesógenos ${ }^{7}$. Uma forma de alinhar esses domínios depende da aplicação de uma tensão uniaxial na amostra. Para um intervalo de baixas temperaturas, experimentos mostram uma curva peculiar da tensão nominal como função da deformação, com três regiões características (Figura 5.6a). Para pequenas deformações, o sistema se encontra na fase de polidomínios, a amostra é opaca e a tensão varia linearmente com a deformação de acordo com a lei de Hooke. Na segunda região, a tensão permanece constante para um intervalo de valores intermediários da deformação, e o parâmetro de ordem nemático apresenta um aumento significativo (Figura 5.6b). Após o plateau o estado de monodomínio é alcançado, a tensão volta a crescer com a deformação, e a amostra é opticamente transparente (Clarke, Terentjev, Kundler, e Finkelmann, 1998; Ortiz, Ober, e Kramer, 1998; Ortiz, Wagner, Bhargava, Ober, e Kramer, 1998). Alguns autores sugerem que a condição de formação das conexões cruzadas, modelada pelo termo de interação com campos aleatórios, é essencial para explicar o estado estável de polidomínios (Fridrikh e Terentjev, 1999; Uchida, 2000). Em particular, Fridrikh e Terentjev incorporaram campos aleatórios à teoria neoclássica da elasticidade para obter um bom ajuste de vários resultados experimentais (Fridrikh e Terentjev, 1999).

A etapa de formação das conexões cruzadas, em particular o contraste entre os resultados obtidos para amostras sintetizadas nos estados nemático e isotrópico, foi investigada por um trabalho experimental recente (Urayama, Kohmon, Kojima, e Takigawa, 2009). Conclusões gerais apontam para um plateau bem-definido na curva tensão versus deformação, a tensões críticas razoavelmente baixas, para amostras formadas no estado isotrópico (Figura 5.7a), contrastando com os resultados obtidos para amostras formadas no estado nemático (Figura 5.7b). Como veremos na seção 5.6, nosso modelo provê uma explicação qualitativa para esses resultados.

Nossa abordagem possui a vantagem de proporcionar uma maneira simples de investigar um amplo espectro de parâmetros, inclusive o papel de campos aleatórios, assim como fornecer uma visão unificada do comportamento crítico de ENs, em concordância

\footnotetext{
${ }^{7}$ A textura Schlieren também pode ser observada como um efeito transiente, rapidamente termalizado, em nemáticos usuais (Warner e Terentjev, 2003). Apenas no ínicio da década de 90 os experimentais tiveram sucesso em produzir elastômeros nemáticos na fase monodomínio sem a necessidade de aplicação de uma tensão externa (Küpfer e Finkelmann, 1991).
} 

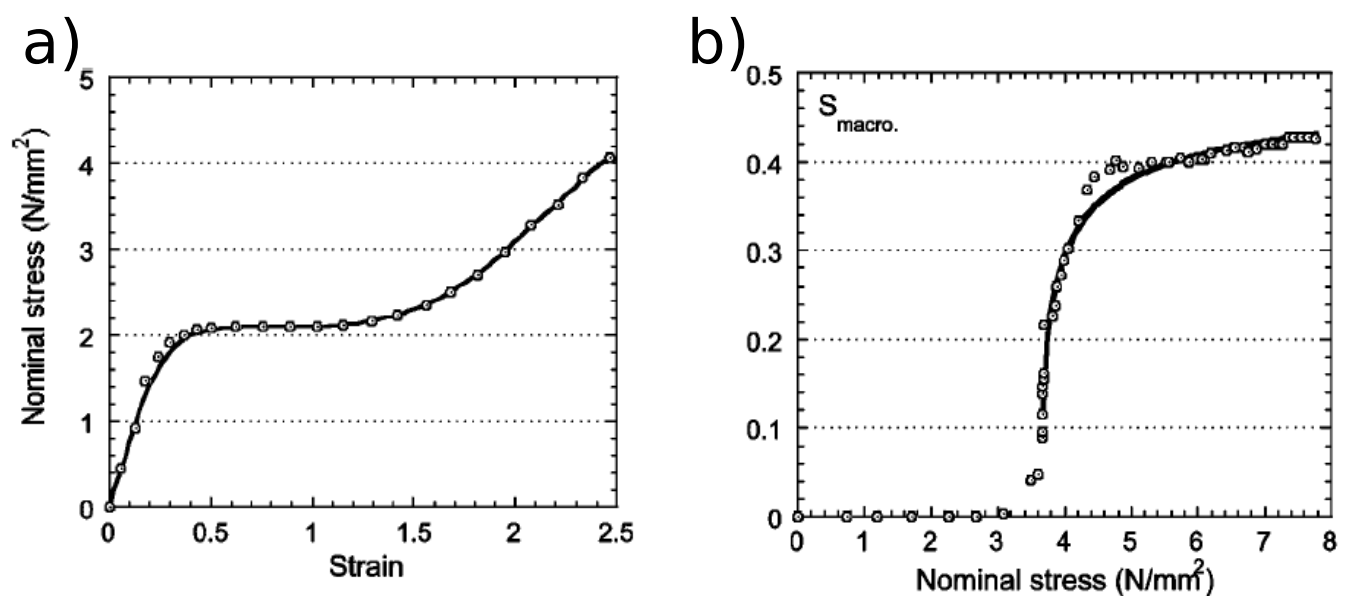

Figura 5.6: Curvas experimentais para elastômeros nemáticos. (a) Tensão nominal como função da deformação. (b) Parâmetro de ordem nemático como função da tensão nominal. Fonte: (Fridrikh e Terentjev, 1999).
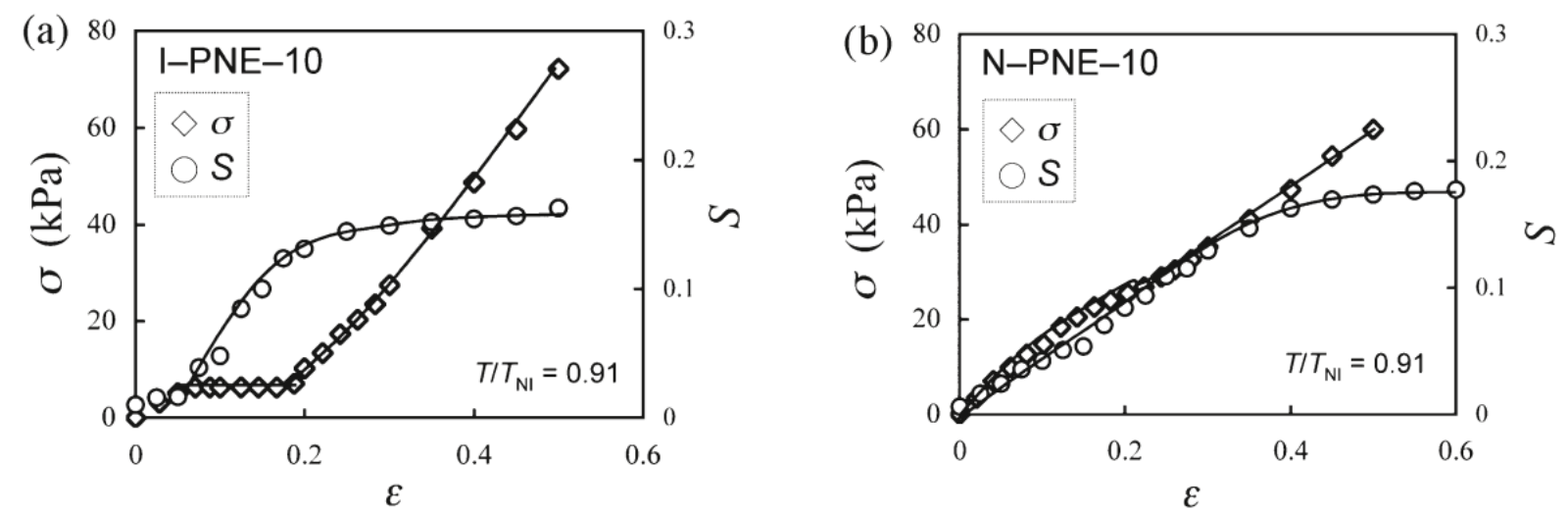

Figura 5.7: Resultados experimentais para a tensão $\sigma$ e o parâmetro de ordem nemático $S$ como função da deformação $\epsilon$ para uma amostra formada no estado isotrópico (a) e nemático (b). Fonte: (Urayama et al., 2009).

qualitativa com trabalhos anteriores.

\subsection{Modelo de rede para um elastômero nemático}

Como vimos no capítulo 4, os elementos básicos do modelo de Maier-Saupe são vetores unitários $\boldsymbol{n}_{i}$, representando um conjunto de $N$ mesógenos, que interagem via a energia de 
quadrupolo

$$
E_{\mathrm{MS}}=-\frac{A}{N} \sum_{1 \leq i<j \leq N} \sum_{\mu, \nu=x, y, z} S_{i}^{\mu \nu} S_{j}^{\mu \nu}
$$

onde $A>0$ é uma constante de energia,

$$
S_{i}^{\mu \nu}=\frac{1}{2}\left(3 n_{i}^{\mu} n_{i}^{\nu}-\delta^{\mu \nu}\right)
$$

e $\left|\boldsymbol{n}_{i}\right|=1$ para $i=1,2, \cdots, N$. Os cálculos se tornam ainda mais simples se adotarmos a sugestão de Zwanzig (Zwanzig, 1963) e restringirmos o número de orientacões permitidas de $\boldsymbol{n}_{i}$ aos seis valores sobre os eixos cartesianos,

$$
\boldsymbol{n}_{i} \in\{( \pm 1,0,0),(0, \pm 1,0),(0,0, \pm 1)\}
$$

Generalizações deste modelo, que chamamos modelo de Maier-Saupe-Zwanzig (MSZ), foram aplicadas ao estudo dos ordenamentos nemáticos uniaxiais e biaxiais de cristais líquidos (Henriques e Henriques, 1997; Carmo et al., 2010). Como previsto pela teoria de Landau e de Gennes (Gennes e Prost, 1993), a transição entre as fases nemática e isotrópica é de primeira ordem, com uma descontinuidade no parâmetro de ordem nemático. No caso de ENs, Xing e colaboradores consideraram interações semelhantes, do tipo Maier-Saupe, num modelo microscópico que tenta estabelecer contato com a teoria macroscópica de Landau (Xing, Pfahl, Mukhopadhyay, Goldbart, e Zippelius, 2008).

Várias abordagens tem sido usadas para modelar o caráter mecânico e orientacional de elastômeros nemáticos (Golubović e Lubensky, 1989; Uchida, 2000; Warner e Terentjev, 2003; Selinger e Ratna, 2004; Pasini, Skačej, e Zannoni, 2005; Ennis, Malacarne, PalffyMuhoray, e Shelley, 2006; Jayasri et al., 2009). Vamos admitir que as propriedades elásticas são geradas por contribuições puramente entrópicas (Warner e Terentjev, 2003). Consideramos então a função canônica de partição

$$
Z=\sum_{\left\{\boldsymbol{n}_{i}\right\}} \Omega\left(\left\{\boldsymbol{n}_{i}\right\}, \Lambda\right) \exp \left(-\beta E_{\mathrm{MS}}\right)
$$

onde $\beta=1 / k T, E_{\mathrm{MS}}$ é a energia de interação do modelo MSZ, dada pela equação (5.1), e a soma é sobre as configurações (5.3) dos diretores nemáticos $\boldsymbol{n}_{i}$. O termo entrópico $\Omega$ depende das orientações dos diretores locais e de um tensor de distorção global da rede $\Lambda$ (Warner e Terentjev, 2003). Para deformações uniformes ao longo da direção de um 
vetor unitário $\boldsymbol{m}$, as componentes cartesianas de $\boldsymbol{\Lambda}$ podem ser escritas na forma

$$
\Lambda_{\alpha \beta}=\lambda^{-1 / 2} \delta_{\alpha \beta}+\left(\lambda-\lambda^{-1 / 2}\right) m_{\alpha} m_{\beta}, \quad \alpha, \beta=x, y, z,
$$

onde $\lambda$ é o fator de distorção e admitidos incompressibilidade da amostra. De acordo com uma extensão da teoria neoclássica da elasticidade (Warner e Terentjev, 1996, 2003) para hamiltonianas de rede, proposta por Selinger e Ratna (Selinger e Ratna, 2004), escrevemos o fator de degenerescência $\Omega$ como

$$
\Omega=\exp \left(-\beta F_{\mathrm{el}}\right)
$$

com a energia livre elástica

$$
F_{\mathrm{el}}=\frac{\mu}{2} \sum_{i} \operatorname{Tr}\left(\boldsymbol{l}_{0, i} \cdot \boldsymbol{\Lambda}^{T} \cdot \boldsymbol{l}_{i}^{-1} \cdot \boldsymbol{\Lambda}\right),
$$

onde $\mu$ é o módulo linear de cisalhamento, $\boldsymbol{l}_{i}$ é o tensor de forma local, e $\boldsymbol{l}_{0, i}$ é o tensor de forma local no instante de formação das conexões cruzadas. As componentes dos tensores de forma são obtidas da equação

$$
l_{i, \alpha \beta}^{-1}=l_{\perp}^{-1} \delta_{\alpha \beta}+\left(l_{\|}^{-1}-l_{\perp}^{-1}\right) n_{i, \alpha} n_{i, \beta},
$$

onde $l_{\perp}$ e $l_{\|}$são os comprimentos de passos efetivos dos polímeros nemáticos nas direções perpendicular e paralela com relação aos vetores nemáticos. Se a rede de conexões cruzadas é formada com a amostra num estado completamente isotrópico ${ }^{8}$, admitimos que o tensor de forma $\boldsymbol{l}_{0, i}^{-1}$ é dado por um média isotrópica de $\boldsymbol{l}_{i}^{-1}$,

$$
l_{0, \alpha \beta}^{-1}=\frac{1}{3}\left(2 l_{\perp}^{-1}+l_{\|}^{-1}\right) \delta_{\alpha \beta} .
$$

Assim

$$
\begin{aligned}
F_{\mathrm{el}} & =\frac{\mu}{2} \sum_{i=1}^{N}\left[\left(\lambda^{2}+2 \lambda^{-1}\right)-\delta\left(\lambda^{2}-\lambda^{-1}\right)\left(\frac{3}{2}\left(\boldsymbol{m} \cdot \boldsymbol{n}_{i}\right)^{2}-\frac{1}{2}\right)\right] \\
& =\frac{\mu N}{2}\left(\lambda^{2}+\frac{2}{\lambda}\right)-\frac{\mu \delta}{3}\left(\lambda^{2}-\frac{1}{\lambda}\right) \sum_{i=1}^{N} \sum_{\mu, \nu} M_{\mu \nu} S_{i}^{\mu \nu}
\end{aligned}
$$

onde introduzimos o tensor

$$
M_{\mu \nu}=\frac{1}{2}\left(3 m^{\mu} m^{\nu}-\delta^{\mu \nu}\right),
$$

\footnotetext{
${ }^{8}$ Podemos incorporar o caso de amostras formadas no caso nemático considerando interações com campos aleatórios.
} 
e o parâmetro

$$
\delta=\frac{2 l_{\perp}^{-1}-2 l_{\|}^{-1}}{2 l_{\perp}^{-1}+l_{\|}^{-1}}, \quad 0 \leq \delta \leq 1,
$$

com $\delta=0$ no caso isotrópico e $\delta=1$ no limite de anisotropia máxima. O primeiro termo no lado direito da equação (5.10) é a energia livre clássica da borracha,

$$
f_{\mathrm{rub}}=\frac{\mu}{2}\left(\lambda^{2}+\frac{2}{\lambda}\right)
$$

A função de partição pode ser escrita então como

$$
Z=\sum_{\left\{\boldsymbol{n}_{i}\right\}} \exp \left(-\beta E_{\mathrm{eff}}\right)
$$

com a energia efetiva

$$
E_{\mathrm{eff}}=-\frac{A}{N} \sum_{1 \leq i<j \leq N} \sum_{\mu, \nu=x, y, z} S_{i}^{\mu \nu} S_{j}^{\mu \nu}-\frac{4}{9} B \sum_{i=1}^{N} \sum_{\mu, \nu=x, y, z} M_{\mu \nu} S_{i}^{\mu \nu}+N f_{\mathrm{rub}}
$$

onde definimos

$$
B=\frac{3}{4} \mu \delta\left(\lambda^{2}-\frac{1}{\lambda}\right)
$$

Dada a origem entrópica da contribuição elástica à energia livre, o módulo linear de cisalhamento deve depender linearmente da temperatura,

$$
\mu=n_{\mathrm{s}} k_{\mathrm{B}} T
$$

onde $n_{\mathrm{s}}$ é o número de filamentos na rede de polímeros por unidade de volume (Warner e Terentjev, 2003).

Admitimos ainda que os diretores nemáticos interagem com campos aleatórios, originados da distribuição de eixos de anisotropia gerados pela formação de conexões cruzadas (Figura 5.8). Essas interações podem ser representadas pelo termo de energia (Fridrikh e Terentjev, 1999; Golubović e Lubensky, 1989; Fridrikh e Terentjev, 1997; Yu et al., 1998)

$$
E_{\mathrm{rf}}=-\frac{\gamma}{2} \sum_{i=1}^{N} \sum_{\mu, \nu=x, y, z} H_{i}^{\mu \nu} S_{i}^{\mu \nu}
$$

com

$$
H_{i}^{\mu \nu}=\frac{1}{2}\left(3 h_{i}^{\mu} h_{i}^{\nu}-\delta^{\mu \nu}\right)
$$


onde $\gamma$ é um parâmetro de energia, e $h_{i}^{\mu}$ é a $\mu$-ésima componente do vetor unitário $\boldsymbol{h}_{i}$. Admitimos que $\left\{\boldsymbol{h}_{i}\right\}$ é um conjunto de variáveis aleatórias temperadas, independentes e identicamente distribuídas com distribuição de probabilidade

$$
P(\boldsymbol{h})= \begin{cases}c / 2, & \text { para } \boldsymbol{h}=(0,0, \pm 1), \\ (1-c) / 4, & \text { para } \boldsymbol{h}=( \pm 1,0,0),(0, \pm 1,0),\end{cases}
$$

com $0 \leq c \leq 1$. Escolhemos o eixo de simetria dos mesógenos ao longo da direção z. Assim o parâmetro $c$ está relacionado ao grau de anisotropia dos mesógenos anexados às conexões cruzadas (ou às próprias conexões cruzadas), tal que $c=1 / 3$ para amostras formadas na fase isotrópica, e $c>1 / 3$ para amostras formadas na fase nemática. Note que admitimos o tensor de forma $\boldsymbol{l}_{0, i}^{-1}$ fixo como uma média isotrópica de $\boldsymbol{l}_{i}^{-1}$, e escolhemos incluir efeitos de memória da rede apenas nas interações com os campos aleatórios.

a)

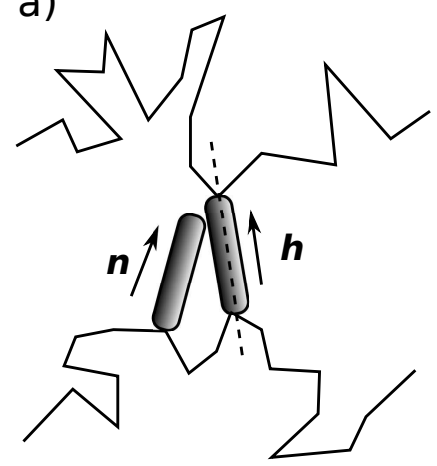

b)

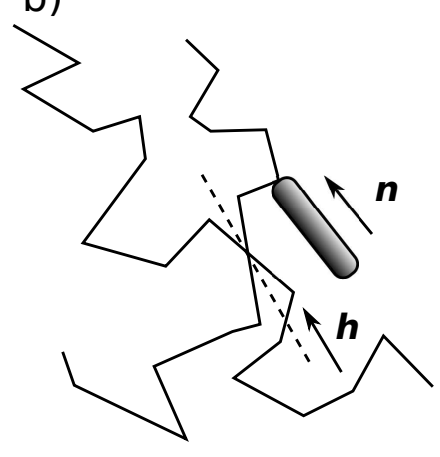

Figura 5.8: Conexões cruzadas geram eixos de anisotropia (linhas tracejadas) que forçam um alinhamento dos diretores nemáticos locais em ambos os casos: (a) com um mesógeno fazendo a ponte entre duas cadeias poliméricas; (b) sem a presença de mesógenos no ponto de junção.

\subsection{Cálculo da energia livre}

Efeitos de tensões externas fixas $\sigma$ podem ser incorporados por meio da função de partição

$$
Y\left(\left\{\boldsymbol{h}_{i}\right\}\right)=\int_{0}^{\infty} d \lambda e^{N \beta \sigma \lambda} \sum_{\left\{\boldsymbol{n}_{i}\right\}} \exp \left[-\beta\left(E_{\mathrm{eff}}+E_{\mathrm{rf}}\right)\right],
$$


para uma dada configuração de campos aleatórios. A densidade de energia livre é dada por

$$
f=-\frac{1}{\beta} \lim _{N \rightarrow \infty} \frac{1}{N} \ln Y=f_{\text {rub }}-\sigma \lambda-\frac{1}{\beta} \lim _{N \rightarrow \infty} \frac{1}{N} \ln \sum_{\left\{\boldsymbol{n}_{i}\right\}} \exp (-\beta E),
$$

onde $f$ deve ser minimizada com relação a $\lambda$ e

$$
E=-\frac{A}{2 N} \sum_{\mu, \nu}\left(\sum_{i=1}^{N} S_{i}^{\mu \nu}\right)^{2}-\frac{4}{9} B \sum_{i=1}^{N} \sum_{\mu, \nu} M_{\mu \nu} S_{i}^{\mu \nu}-\frac{\gamma}{2} \sum_{\mu, \nu} \sum_{i=1}^{N} H_{i}^{\mu \nu} S_{i}^{\mu \nu}
$$

onde descartamos termos de ordem menor que $N$.

Com o auxílio de identidades gaussianas podemos desacoplar as interações entre as partículas,

$$
\begin{aligned}
\sum_{\left\{\boldsymbol{n}_{i}\right\}} \exp (-\beta E)= & \int[d Q] \exp \left(-\frac{N \beta A}{2} \sum_{\mu, \nu} Q_{\mu \nu}^{2}\right) \\
& \times \prod_{i}\left\{\sum_{\{\boldsymbol{n}\}} \exp \left[\beta \sum_{\mu, \nu} S_{i}^{\mu \nu}\left(A Q_{\mu \nu}+\frac{4}{9} B M_{\mu \nu}+\frac{\gamma}{2} H_{i}^{\mu \nu}\right)\right]\right\}
\end{aligned}
$$

onde $[d Q]=\prod_{\mu \nu} \sqrt{\beta A N / 2 \pi} d Q_{\mu \nu}$. Após calcular a soma sobre as orientações $\boldsymbol{n}$ de uma única partícula, obtemos

$$
\begin{aligned}
f= & f_{\text {rub }}+\frac{B}{3}-\sigma \lambda-\frac{1}{\beta} \ln 2-\beta^{-1} \lim _{N \rightarrow \infty} \frac{1}{N} \ln \int[d Q] \exp \left[-\frac{N \beta A}{2}\left(\sum_{\mu, \nu} Q_{\mu \nu}^{2}+\operatorname{Tr} \mathbb{Q}\right)\right] \\
& \times \exp \left\{\sum_{i=1}^{N}\left[-\frac{\beta \gamma}{4} \operatorname{Tr} \mathbb{H}_{i}+\ln \left(\sum_{\mu} e_{\mu}\left(\boldsymbol{h}_{i}\right)\right)\right]\right\}
\end{aligned}
$$

onde

$$
e_{\mu}\left(\boldsymbol{h}_{i}\right)=\exp \left[\beta\left(\frac{3 A}{2} Q_{\mu \mu}+\frac{3 \gamma}{4} H_{i}^{\mu \mu}+B m_{\mu}^{2}\right)\right] .
$$

De acordo com a lei dos grandes números,

$$
\lim _{N \rightarrow \infty} \frac{1}{N} \sum_{i=1}^{N}\left\{-\frac{\beta \gamma}{4} \operatorname{Tr} \mathbb{H}_{i}+\ln \left(\sum_{\mu} e_{\mu}\left(\boldsymbol{h}_{i}\right)\right)\right\}=-\frac{\beta \gamma}{4}\langle\operatorname{Tr} \mathbb{H}\rangle_{h}+\left\langle\ln \left(\sum_{\mu} e_{\mu}(\boldsymbol{h})\right)\right\rangle_{h}
$$

onde $\langle\ldots\rangle_{h}$ denota o valor esperado com relação às variáveis de campo aleatório, de onde vemos que a energia livre é auto-mediante. Utilizamos então o método de Laplace, de forma que

$$
f=f_{\mathrm{rub}}+\frac{B}{3}-\sigma \lambda-\frac{1}{\beta} \ln 2-\beta^{-1} \max L\left(Q_{\mu \nu}\right),
$$


onde $Q_{\mu \nu}$ maximiza o funcional

$$
L=-\frac{\beta A}{2}\left(\operatorname{Tr} \mathbb{Q}^{2}+\operatorname{Tr} \mathbb{Q}\right)+\left\langle\ln \left(\sum_{\mu} e_{\mu}\right)\right\rangle_{h} .
$$

A condição de $L$ ser um ponto estacionário com relação a $Q_{\mu \nu}$ leva às equações de estado para os parâmetros de ordem,

$$
Q_{\mu \nu}=\frac{1}{2}\left(3\left\langle\frac{e_{\mu}}{\sum_{\alpha} e_{\alpha}}\right\rangle_{h}-1\right) \delta_{\mu \nu}
$$

Note que $\operatorname{Tr} \mathbb{Q}=0$. A condição para a energia livre ser estacionária com relação a $\lambda$ leva à equação de estado para a distorção,

$$
\lambda=\frac{1}{\lambda^{2}}+\frac{\sigma}{\mu}+\frac{\delta}{2}\left(2 \lambda+\frac{1}{\lambda^{2}}\right) \sum_{\mu} m_{\mu}^{2} Q_{\mu \mu},
$$

onde usamos o resultado (5.30). Essas equações podem ser usadas para reescrever a densidade de energia livre como

$$
f=f_{\mathrm{rub}}+\frac{B}{3}-\sigma \lambda-\frac{1}{\beta} \ln 2+\frac{A}{2} \operatorname{Tr} \mathbb{Q}^{2}-\frac{1}{\beta}\left\langle\ln \left(\sum_{\mu} e_{\mu}\right)\right\rangle_{h} .
$$

Para estabelecer contato mais próximo com experimentos em cristais líquidos, usamos a representação paramétrica diagonal padrão da matriz de traço nulo $\mathbb{Q}$ apropriada para ordenamento nemático ao longo do eixo $z$,

$$
\mathbb{Q}=\left(\begin{array}{ccc}
-\frac{S+\eta}{2} & 0 & 0 \\
0 & -\frac{S-\eta}{2} & 0 \\
0 & 0 & S
\end{array}\right)
$$

Os parâmetros de ordem $S$ e $\eta$ caracterizam a fase isotrópica $(S=\eta=0)$, nemática uniaxial $(S \neq 0, \eta=0)$ e nemática biaxial $(S \neq 0, \eta \neq 0)$. Das equações de estado (5.30) obtemos

$$
S=\frac{3}{2}\left\langle\frac{e_{z}}{\sum_{\mu} e_{\mu}}\right\rangle_{h}-\frac{1}{2}
$$

$\mathrm{e}$

$$
\eta=\frac{3}{2}\left\langle\frac{e_{y}-e_{x}}{\sum_{\mu} e_{\mu}}\right\rangle_{h}
$$

A versão mais simples do modelo MSZ definido pelas equações (5.1-5.3) não possui a simetria necessária para descrever uma fase nemática biaxial estável. Para verificar 
se este comportamento é robusto após a inclusão de elasticidade e campos aleatórios, consideramos o caso onde a deformação é perpendicular ao eixo de simetria escolhido do parâmetro de ordem. De fato, tem havido vários debates na literatura sobre a origem de um comportamento suave (ou semisuave) estar relacionada ao início de um ordenamento biaxial (Warner e Terentjev, 2003; Verwey, Warner, e Terentjev, 1996; Warner e Kutter, 2002; Ye e Lubensky, 2009). Enquanto a descrição deste fenômeno está além dos objetivos deste trabalho, enfatizamos que uma inspeção numérica das equações de estado nos leva a concluir que a simetria biaxial permanece ausente para um amplo intervalo de parâmetros. Assim tomamos $\eta=0$ e consideramos a direção da deformação paralela ao eixo $z, \boldsymbol{m}=$ $(0,0,1)$. Essa escolha é razoável desde que o acoplamento entre graus de liberdade elásticos e orientacionais fornecem um eixo preferencial de simetria para esse sistema. A densidade de energia livre é dada então por

$$
f=f_{\mathrm{rub}}+\frac{1}{3} B-\sigma \lambda-\frac{1}{\beta} \ln 2+\frac{3 A}{4} S^{2}-\frac{1}{\beta}\left\langle\ln \left(\sum_{\mu} e_{\mu}\right)\right\rangle_{h},
$$

onde

$$
\begin{aligned}
& e_{\mu}(\boldsymbol{h})=\exp \left\{\beta\left[-\frac{3}{4} A S+\frac{3}{8} \gamma\left(3 h_{\mu}^{2}-1\right)\right]\right\}, \quad \mu=x, y, \\
& e_{z}(\boldsymbol{h})=\exp \left\{\beta\left[\frac{3}{2} A S+\frac{3}{8} \gamma\left(3 h_{z}^{2}-1\right)+B\right]\right\},
\end{aligned}
$$

e a equação de estado para a distorção (5.31) tem a forma

$$
\lambda=\frac{1}{\lambda^{2}}+\frac{\sigma}{\mu}+\frac{\delta}{2}\left(2 \lambda+\frac{1}{\lambda^{2}}\right) S
$$

Até agora os resultados obtidos são válidos para qualquer distribuição de campos aleatórios. Vamos nos limitar daqui por diante à distribuição discreta (5.20). Neste caso a densidade de energia livre (5.36) é dada por

$$
\begin{aligned}
f= & f_{\text {rub }}+\frac{1}{3} B-\sigma \lambda-\frac{1}{\beta} \ln 2+\frac{3}{4} A S(S+1) \\
& -\frac{1}{\beta}\left\{c \ln \left[2+e^{\beta(9 A S / 4+9 \gamma / 8+B)}\right]+(1-c) \ln \left[1+e^{9 \beta \gamma / 8}+e^{\beta(9 A S / 4+B)}\right]\right\},
\end{aligned}
$$

e a equação de estado para o parâmetro de ordem (5.34) resulta

$$
S=\frac{3}{2}\left[\frac{c e^{\beta(9 A S / 4+9 \gamma / 8+B)}}{2+e^{\beta(9 A S / 4+9 \gamma / 8+B)}}+\frac{(1-c) e^{\beta(9 A S / 4+B)}}{1+e^{9 \beta \gamma / 8}+e^{\beta(9 A S / 4+B)}}\right]-\frac{1}{2} .
$$




\subsection{Resultados termodinâmicos}

Sem perda de generalidade, admitimos que $n_{\mathrm{s}}=1$ e $\delta=0.5$, uma vez que outras escolhas levam a resultados qualitativamente semelhantes. Vamos escrever agora as equações que utilizamos nos cálculos numéricos. Expressamos as energias em unidades de $A$ e a temperatura em unidades de $k / A$. A densidade de energia livre (5.41) é dada explicitamente por

$$
\begin{aligned}
f= & \frac{3}{4} S(S+1)+T\left\{\frac{1}{8}\left(5 \lambda^{2}-7 \lambda^{-1}\right)-\ln 2-c \ln \left[2+e^{9(2 S+\gamma) / 8 T+3\left(\lambda^{2}-\lambda^{-1}\right) / 8}\right]\right. \\
& \left.-(1-c) \ln \left[1+e^{9 \gamma / 8 T}+e^{9 S / 4 T+3\left(\lambda^{2}-\lambda^{-1}\right) / 8}\right]\right\} .
\end{aligned}
$$

A equação de estado para o parâmetro de ordem (5.41) toma a forma

$$
S=\frac{3}{2}\left[\frac{c e^{9(2 S+\gamma) / 8 T+3\left(\lambda^{2}-\lambda^{-1}\right) / 8}}{2+e^{9(2 S+\gamma) / 8 T+3\left(\lambda^{2}-\lambda^{-1}\right) / 8}}+\frac{(1-c) e^{9 S / 4 T+3\left(\lambda^{2}-\lambda^{-1}\right) / 8}}{1+e^{9 \gamma / 8 T}+e^{9 S / 4 T+3\left(\lambda^{2}-\lambda^{-1}\right) / 8}}\right]-\frac{1}{2},
$$

e a equação de estado para a distorção (5.39) resulta

$$
\lambda=\frac{1}{\lambda^{2}}+\frac{\sigma}{T}+\frac{1}{4}\left(2 \lambda+\frac{1}{\lambda^{2}}\right) S
$$

Na ausência de tensões aplicadas $(\sigma=0)$, a equação de estado (5.44) pode ser facilmente resolvida para a distorção, com o resultado

$$
\lambda=\left(\frac{1+S / 4}{1-S / 2}\right)^{\frac{1}{3}} .
$$

A distorção aumenta monotonicamente $\operatorname{com} S$, para $0<S<1$. Além disso, $S=0$ implica $\lambda=1$, indicando que a deformação é induzida pelo ordenamento orientacional apenas. Para uma tensão aplicada arbitrária $(\sigma \geq 0)$, a equação de estado (5.44) é uma equação cúbica em $\lambda$ com apenas uma raiz real e positiva dada pela fórmula ${ }^{9}$

$$
\lambda=\frac{2 \sigma}{3(2-S) T}\left\{1+2 \cosh \left[\frac{1}{3} \cosh ^{-1}\left(1+\frac{27(2-S)^{2}(4+S) T^{3}}{32 \sigma^{3}}\right)\right]\right\},
$$

que é uma função monótona crescente de $S$. Em nossos cálculos numéricos, resolvemos a equação (5.43) para o parâmetro de ordem $S$ usando para $\lambda$ o resultado dado pela equação (5.46).

\footnotetext{
${ }^{9}$ Veja o apêndice B para uma resolução sucinta da equação cúbica.
} 


\subsubsection{Resultados na ausência de desordem $(\gamma=0)$}

Na Figura 5.9 traçamos o parâmetro de ordem $S$ e a densidade de energia livre $f$ (curva superior) como uma função da temperatura para tensões aplicadas $\sigma=0.02$, no intervalo de temperaturas onde ocorre uma transição de primeira ordem. A solução estável de baixas temperaturas abc, com valores mais altos do parâmetro de ordem $S$, será chamada solução nemática, enquanto a solução estável de altas temperaturas dbe, com valor mais baixo do parâmetro de ordem, será chamada solução isotrópica, mesmo quando $S$ não é necessariamente nulo, como no caso onde há tensões aplicadas. O ramo cd corresponde a uma solução instável. No intervalo de temperaturas $T_{d}<T<T_{c}$ ambas as soluções nemática e isotrópica são estáveis, e temos que escolher aquela com o menor valor da energia livre. A transição de primeira ordem ocorre na temperatura $T_{\mathrm{NI}}=T_{b}$ onde as energias se tornam iguais. Observamos que, em concordância com a teoria elástica clássica, a energia livre varia linearmente com a temperatura na fase isotrópica (Warner e Terentjev, 2003).

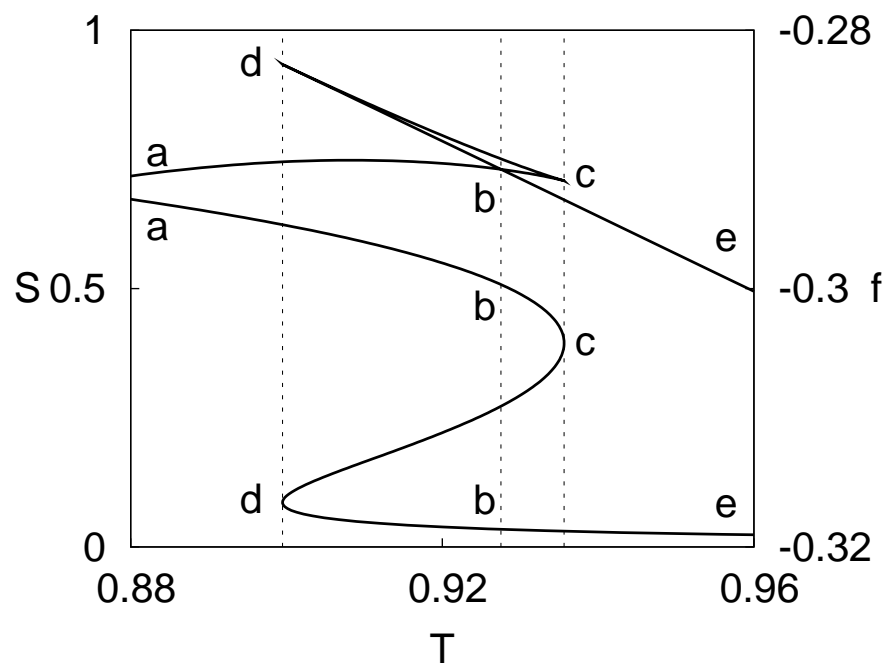

Figura 5.9: Parâmetro de ordem $S$ (curva inferior) e densidade de energia livre $f$ (curva superior) como funções da temperatura para $\gamma=0$ e tensão aplicada $\sigma=0.02$.

Na Figura 5.10 apresentamos o parâmetro de ordem nemático $S$ e o fator de distorção $\lambda$ como funções da temperatura para vários valores da tensão aplicada $\sigma$. Como se espera 
da equação (5.46), o gráfico de $\lambda$ é semelhante ao de $S$. Para valores pequenos da tensão aplicada $\sigma$, o sistema sofre uma transição de primeira ordem com uma separação entre as soluções nemática e isotrópica. À medida que a tensão $\sigma$ aumenta, essa separação diminui até um ponto crítico ser atingido, a partir do qual não existe transição de fase. Este comportamento foi predito por De Gennes em meados da década de 70 (Gennes, 1975), antes mesmo da consistência química de elastômeros nemáticos ser demonstrada. Experimentalmente, entretanto, não há transições de fase de primeira ordem associadas mesmo na ausência de tensões externas. O salto na transição de primeira ordem é suavizado, sendo substituído por uma variação contínua, embora rápida, do parâmetro de ordem. Este fato tem sido interpretado teoricamente com o auxílio de distribuições anisotrópicas de campos aleatórios (Petridis e Terentjev, 2006; Selinger e Ratna, 2004).
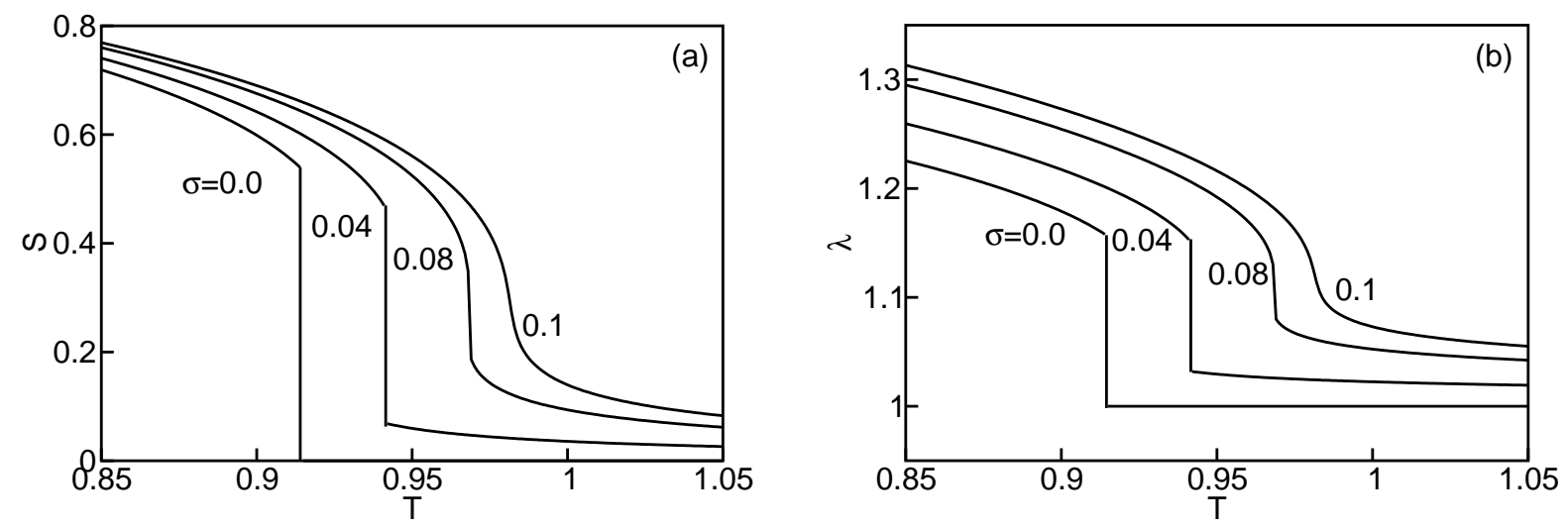

Figura 5.10: Parâmetro de ordem nemático (a) e fator de distorção (b) como função da temperatura, para $\gamma=0$ e vários valores da tensão aplicada $\sigma$.

\subsubsection{Efeitos da desordem $(\gamma>0)$}

Examinaremos agora como a interação com campos aleatórios afeta a transição entre as fases nemática e isotrópica. Na Figura 5.11a mostramos o parâmetro de ordem nemático como uma função da temperatura para $\sigma=0, \gamma=0.2$ e vários valores de $c$. Observamos que a separação entre as soluções nemática e isotrópica decresce com o aumento de $c$ de $c=1 / 3$ a $c=0.422$, desaparecendo acima deste valor. Na Figura 5.11b o parâmetro de ordem nemático é apresentado como uma função da temperatura para $c=0.4$ e vários 
valores de $\gamma$. Novamente, a separação entre as soluções nemática e isotrópica diminui à medida que $\gamma$ aumenta de $\gamma=0$ a $\gamma=0.263$, e a transição desaparece acima deste valor de $\gamma$.
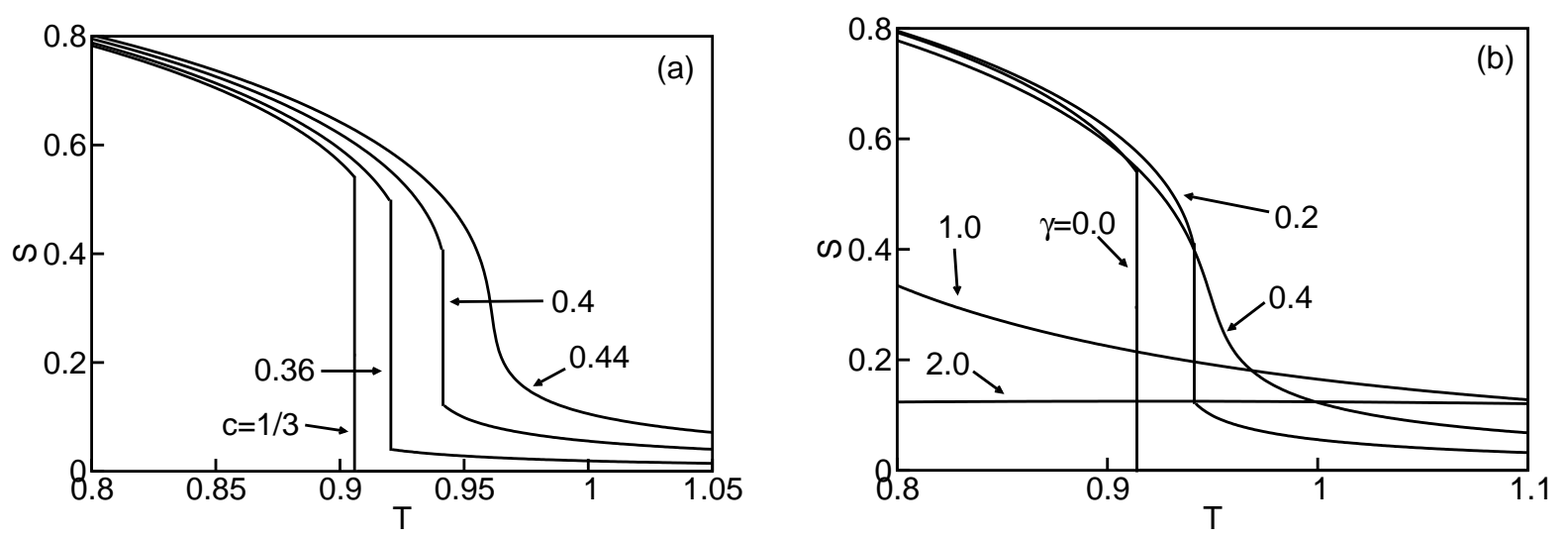

Figura 5.11: Parâmetro de ordem nemático como uma função da temperatura para $\sigma=0$. (a) $\gamma=0.2 \mathrm{e}$ vários valores de $c$. (b) $c=0.4$ e vários valores de $\gamma$.

Esses resultados mostram que uma distribuição anisotrópica $(c>1 / 3)$ de campos aleatórios de intensidade suficiente $(\gamma>0)$ é necessária para suavizar a transição entre as fases nemática e isotrópica, em concordância com as simulações numéricas de Selinger e Ratna (Selinger e Ratna, 2004). A Figura 5.12 mostra, para tensões aplicadas nulas $(\sigma=0)$, a curva no plano $\gamma-c$ a direita da qual não existe transição de primeira ordem.

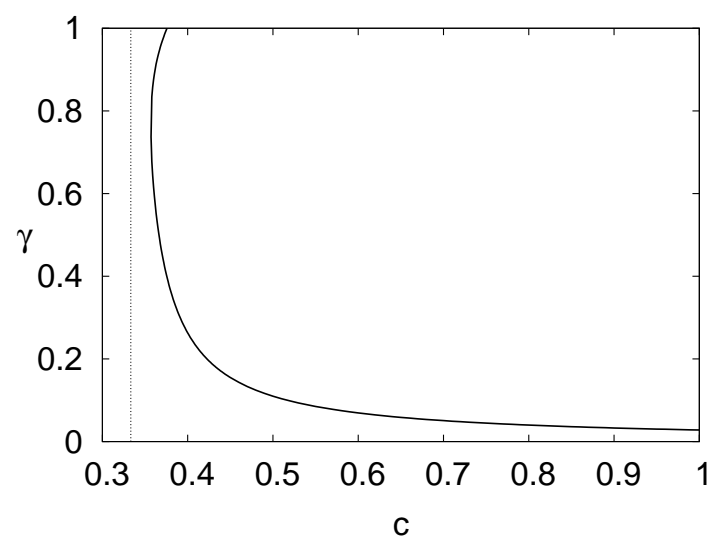

Figura 5.12: A curva para $\sigma=0$ a direita da qual não existe transição de primeira ordem. A linha pontilhada vertical corresponde a $c=1 / 3$. 


\subsubsection{Resultados para desordem isotrópica $(c=1 / 3, \gamma>0)$}

Lembramos que uma distribuição isotrópica de campos aleatórios $(c=1 / 3)$ representa amostras formadas no estado isotrópico, onde as tensões aleatórias originadas da rede com conexões cruzadas não possuem direção preferencial. De acordo com a Figura 5.12, uma transição de primeira ordem ocorre na ausência de tensões aplicadas $(\sigma=0)$ para qualquer valor da intensidade do campo aleatório $\gamma$. Para ilustrar este fato, traçamos na Figura 5.13 o fator de distorção $\lambda$ como uma função da temperatura na ausência de tensões externas $(\sigma=0)$ para vários valores do parâmetro de desordem $\gamma$. A fase nemática decresce com o aumento de $\gamma$, mas a transição de primeira ordem permanece sem mostrar evidência da transição suave entre as fases nemática e isotrópica observada experimentalmente em amostras formadas no estado nemático.

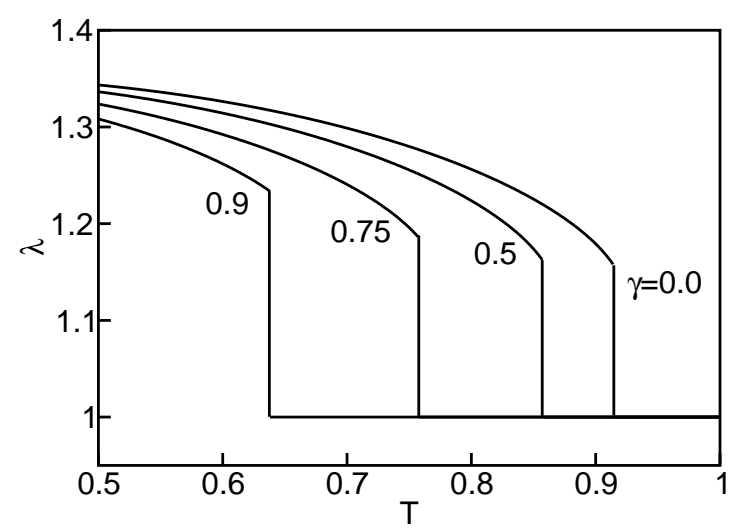

Figura 5.13: Fator de distorção $\lambda$ como uma função da temperatura para $\sigma=0, c=1 / 3$ e $\gamma$ entre 0 e $\approx 1$

Na presença de tensões aplicadas $(\sigma>0)$, a transição de primeira ordem é suavizada para temperaturas $T$ ou parâmetros de desordem $\gamma$ suficientemente altos. Este comportamento pode ser observado nas curvas tensão versus deformação, onde a deformação $e$ está relacionada ao fator de distorção pela equação $\lambda=1+e$. Na Figura 5.14a traçamos isotermas para $\gamma=0.6$ e na Figura 5.14b traçamos curvas iso- $\gamma$ para $T=0.8$. Fica evidente dessas figuras que a temperatura e o parâmetro de desordem tem um efeito similar sobre o sistema. Para temperaturas $\left(T<T_{c}\right)$ ou parâmetro de desordem $\left(\gamma<\gamma_{c}\right)$ suficientemente baixos, a tensão cresce monotonicamente com a deformação na fase isotrópica até um 
ponto onde ocorre uma transição de primeira ordem para a fase nemática. No ponto de transição as duas fases coexistem e a deformação é independente da tensão. O plateau da curva tensão versus deformação é seguido por um aumento da tensão para valores mais altos da deformação. Esse comportamento concorda com o cenário experimental de uma transição típica entre polidomínios e monodomínio em ENs, de acordo com a Figura 5.6a. À medida que o parâmetro de desordem ou a temperatura aumenta, a curva de coexistência se comprime até um ponto crítico ser alcançado em $\gamma=\gamma_{c}$ ou $T=T_{c}$. O diagrama de fases completo lembra um diagrama típico $P-V$ de fluidos simples.
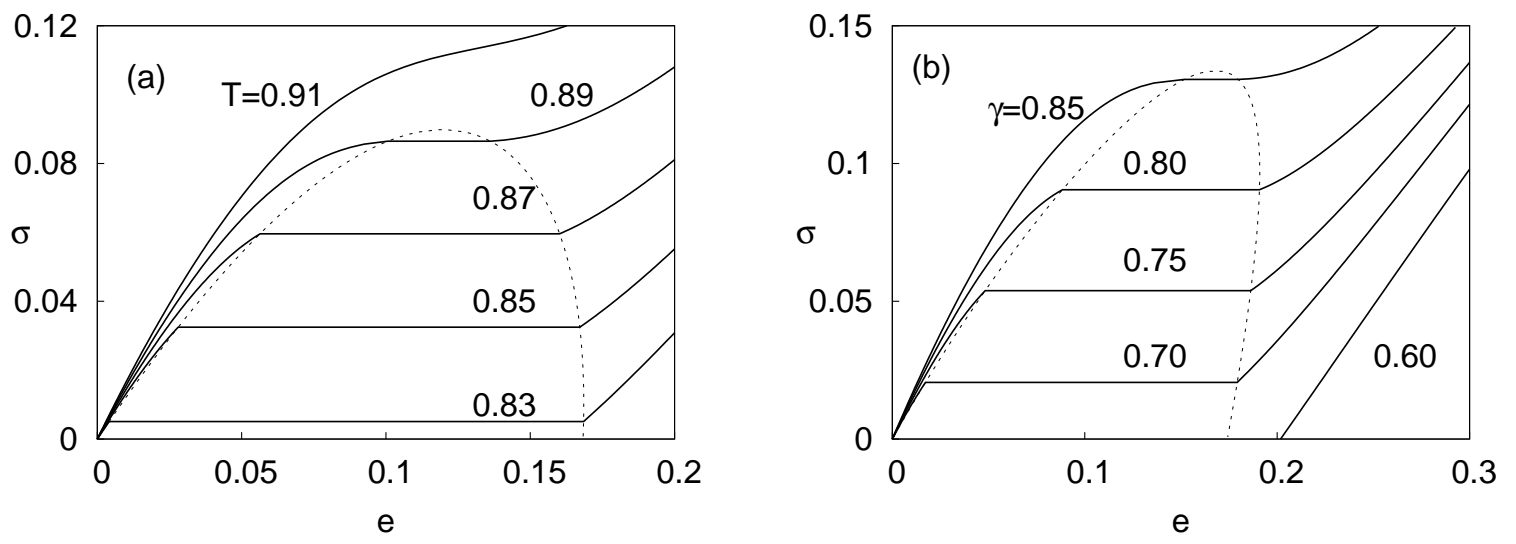

Figura 5.14: Curvas tensão versus deformação para $c=1 / 3$. (a) Isotermas para $\gamma=0.6$. (b) Curvas iso- $\gamma$ para $T=0.8$.

\subsection{Considerações finais}

Vimos na seção 5.2 que experimentos recentes mostram que ENs com conexões cruzadas formadas no estado isotrópico apresentam um plateau bem-definido na curva tensão versus deformação, para valores de tensão crítica muito menores, em comparação com aqueles formados no estado nemático (Urayama et al., 2009). Observações de microscopia óptica sugerem que esse comportamento está associado a efeitos de memória mais fortes para ENs formados no estado nemático. Esperamos então que $\gamma$ não seja independente, mas que aumente $\operatorname{com} c$, de acordo com a linguagem do nosso modelo. Não é difícil agora achar valores apropriados de $\gamma$ e $c$ que satisfazem essa restrição, e em concordância qualitativa 
com os resultados experimentais. Mostramos na Figura 5.15 duas curvas tensão versus deformação para $T=0.95$. Para a curva (b), consideramos amostras formadas no estado isotrópico $(c=1 / 3)$, que implica ausência de efeitos de memória $(\gamma=0)$. Nesse caso, a curva tensão versus deformação apresenta um plateau bem definido para um valor baixo da tensão crítica. Para a curva (a), consideramos amostras formadas no estado nemático $(c=0.35)$, que deve implicar efeitos de memória mais fortes $(\gamma=0.45)$. Note que a curva tensão versus deformação caracteriza uma transição larga de polidomínios a monodomínio orientacional para valores mais altos da tensão crítica, em boa concordância com os resultados experimentais de Urayama e colaboradores (Urayama et al., 2009), (Figura $5.7)$.

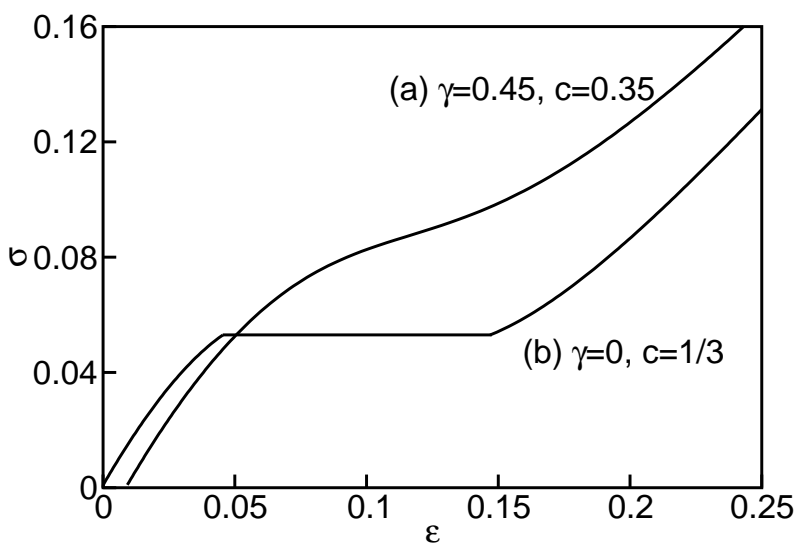

Figura 5.15: Curvas tensão versus deformação para $T=0.95$. (a) $\gamma=0.45$ e $c=0.35$. (b) $\gamma=0$ e $c=1 / 3$.

Para concluir, introduzimos um modelo de rede simples, a nível de campo médio, para descrever o comportamento de elastômeros nemáticos. Este modelo combina a teoria de Maier-Saupe-Zwanzig para cristais líquidos (Maier e Saupe, 1958; Henriques e Henriques, 1997; Carmo et al., 2010) e a versão de rede, devida a Selinger e Ratna (Selinger e Ratna, 2004), da teoria da elasticidade de Warner-Terentjev (Warner e Terentjev, 2003). Realizamos cálculos detalhados para um amplo intervalo de parâmetros, com a inclusão de efeitos de uma distribuição temperada de campos aleatórios. Uma curva de coexistência no gráfico tensão versus deformação pode ser obtida para sistemas resfriados abaixo de uma temperatura de congelamento, que é análoga ao diagrama $P-V$ de um fluido simples, 
com o parâmetro de desordem fazendo o papel da temperatura. Abaixo de uma tensão crítica, as linhas de coexistência lembram o plateau experimental da curva tensão versus deformação, e podem ser interpretadas como assinaturas de uma transição de polidomínios a um monodomínio. No caso de monodomínio, mostramos que campos aleatórios podem suavizar a transição de primeira ordem entre as fases nemática e isotrópica, desde que as amostras tenham sido formadas no estado nemático. Além de concordância geral com resultados anteriores, esperamos que nossos resultados possam motivar trabalhos experimentais adicionais sobre a curva tensão versus deformação de nemáticos elastômeros. 



\section{Apêndice A}

\section{Estabilidade da solução com simetria}

\section{de réplicas}

A matriz hessiana associada ao funcional $-G$, definido em (3.24), possui um total de treze elementos distintos, a saber,

$$
\begin{aligned}
A^{\prime} & =\frac{\partial^{2}(-G)}{\partial x^{\alpha} \partial x^{\alpha}}=2 \beta J b(1-\beta J b)+8 \beta^{2} J^{2} b^{2} \beta J_{0}(a+2 b x) m^{2} q(1-q) \\
& +4 \beta^{4} J^{4} b^{2}(a+2 b x)^{2} q^{2}(1-q)^{2}+4 \beta^{2} J_{0}^{2} b^{2} m^{2}\left(m^{2}-1\right), \\
B^{\prime} & =\frac{\partial^{2}(-G)}{\partial x^{\beta} \partial x^{\alpha}}=-2 \beta^{2} J^{2} b^{2} q^{2}+4 \beta^{2} J_{0}^{2} b^{2} m^{2}\left(m^{2}-q\right)+4 \beta^{4} J^{4} b^{2}(a+2 b x)^{2} q^{2} \\
& \times\left(q^{2}+2 q-3 r\right)+8 \beta^{2} J^{2} b^{2} \beta J_{0}(a+2 b x) m q[2 t-m(1+q)], \\
C^{\prime} & =\frac{\partial^{2}(-G)}{\partial q^{\alpha \beta} \partial x^{\alpha}}=-4 \beta^{2} J^{2} b^{2} \beta J_{0}(a+2 b x) m^{2}(1-q)-4 \beta^{4} J^{4} b^{2}(a+2 b x)^{2} q(1-q)^{2}, \\
D^{\prime} & =\frac{\partial^{2}(-G)}{\partial q^{\beta \gamma} \partial x^{\alpha}}=0, \\
E^{\prime} & =\frac{\partial^{2}(-G)}{\partial m^{\alpha} \partial x^{\alpha}}=2 \beta^{2} J_{0}^{2} b(a+2 b x) m\left(m^{2}-1\right)+2 \beta^{2} J^{2} b \beta J_{0}(a+2 b x)^{2} m q(1-q), \\
F^{\prime} & =\frac{\partial^{2}(-G)}{\partial m^{\beta} \partial x^{\alpha}}=2 \beta^{2} J_{0}^{2} b(a+2 b x) m\left(m^{2}-q\right)+2 \beta^{2} J^{2} b \beta J_{0}(a+2 b x)^{2} q[2 t-m(1+q)], \\
A & =\frac{\partial^{2}(-G)}{\partial m^{\alpha} \partial m^{\alpha}}=\beta J_{0}(a+2 b x)\left[1+\beta J_{0}(a+2 b x)\left(m^{2}-1\right)\right], \\
B & =\frac{\partial^{2}(-G)}{\partial m^{\beta} \partial m^{\alpha}}=\beta^{2} J_{0}^{2}(a+2 b x)^{2}\left(m^{2}-q\right), \\
C & =\frac{\partial^{2}(-G)}{\partial q^{\alpha \beta} \partial m^{\alpha}}=-\beta^{2} J^{2} \beta J_{0}(a+2 b x)^{3} m(1-q), \\
D & =\frac{\partial^{2}(-G)}{\partial q^{\beta \gamma} \partial m^{\alpha}}=-\beta^{2} J^{2} \beta J_{0}(a+2 b x)^{3}(t-m q),
\end{aligned}
$$




$$
\begin{aligned}
P & =\frac{\partial^{2}(-G)}{\partial q^{\alpha \beta} \partial q^{\alpha \beta}}=\beta^{2} J^{2}(a+2 b x)^{2}\left[1-\beta^{2} J^{2}(a+2 b x)^{2}\left(1-q^{2}\right)\right], \\
Q & =\frac{\partial^{2}(-G)}{\partial q^{\alpha \gamma} \partial q^{\alpha \beta}}=-\beta^{4} J^{4}(a+2 b x)^{4} q(1-q) \\
R & =\frac{\partial^{2}(-G)}{\partial q^{\gamma \delta} \partial q^{\alpha \beta}}=-\beta^{4} J^{4}(a+2 b x)^{4}\left(r-q^{2}\right) .
\end{aligned}
$$

onde, no limite $n \rightarrow 0$,

$$
\begin{gathered}
t=\left\langle S^{\alpha} S^{\beta} S^{\gamma}\right\rangle_{L}=\int_{-\infty}^{\infty} \frac{d z}{\sqrt{2 \pi}} \exp \left(-\frac{z^{2}}{2}\right) \tanh ^{3}\left[\beta\left(J \xi q^{1 / 2} z+J_{0} \xi m+H\right)\right], \\
r=\left\langle S^{\alpha} S^{\beta} S^{\gamma} S^{\delta}\right\rangle_{L}=\int_{-\infty}^{\infty} \frac{d z}{\sqrt{2 \pi}} \exp \left(-\frac{z^{2}}{2}\right) \tanh ^{4}\left[\beta\left(J \xi q^{1 / 2} z+J_{0} \xi m+H\right)\right] .
\end{gathered}
$$

Na fase paramagnética

$$
m=q=t=r=0,
$$

de forma que a matriz hessiana é diagonal, com

$$
B^{\prime}=C^{\prime}=D^{\prime}=E^{\prime}=F^{\prime}=B=C=D=Q=R=0
$$

e ainda,

$$
\begin{aligned}
A^{\prime} & =2 \beta J B(1-\beta J b), \\
A & =\beta J_{0}(a+2 b x)\left[1-\beta J_{0}(a+2 b x)\right], \\
P & =\beta^{2} J^{2}(a+2 b x)\left[1-\beta^{2} J^{2}(a+2 b x)^{2}\right],
\end{aligned}
$$

e portanto, a condição de positividade dos autovalores é satisfeita se

$$
\begin{aligned}
& t>b, \\
& t>j_{0} a+b, \\
& t>a+b .
\end{aligned}
$$

As duas últimas condições delimitam a fase paramagnética, que deve ser portanto estável.

Os autovetores da hessiana podem ser escritos na forma

$$
|\mu\rangle=\left(\begin{array}{c}
\left\{\phi^{\alpha}\right\} \\
\left\{\epsilon^{\alpha}\right\} \\
\left\{\eta^{(\alpha \beta)}\right\}
\end{array}\right),
$$


onde os vetores $\phi, \epsilon$ e $\eta$ estão associados às variáveis $x, m$ e $q$, respectivamente. Como no modelo SK, todo o espaço de autovetores linearmente independentes é gerado por três classes de simetria. A primeira, correspondente a autovetores com simetria entre todos os índices,

$$
\phi^{\alpha}=a^{\prime}, \epsilon^{\alpha}=a, \forall \alpha ; \quad \eta^{(\alpha \beta)}=b, \forall \alpha \mathrm{e} \beta,
$$

gera um subespaço com dimensão $3 n$. Na segunda classe,

$$
\begin{aligned}
& \phi^{\alpha}=a^{\prime}, \epsilon^{\alpha}=a \quad \text { para } \quad \alpha=\theta ; \quad \phi^{\alpha}=b^{\prime}, \epsilon^{\alpha}=b, \quad \text { para } \quad \alpha \neq \theta \\
& \eta^{(\alpha \beta)}=c, \quad \text { para } \quad \alpha \quad \text { ou } \quad \beta=\theta ; \quad \eta^{(\alpha \beta)}=d, \quad \text { para } \quad \alpha, \beta \neq \theta .
\end{aligned}
$$

o subespaço gerado também tem dimensão $3 n$. A condição de ortogonalidade com os vetores da primeira classe implica: $a^{\prime}=(1-n) b^{\prime} ; a=(1-n) b ; c=(1-n / 2) d$. A terceira classe de autovetores,

$$
\begin{aligned}
& \phi^{\alpha}=a^{\prime}, \epsilon^{\alpha}=a \quad \text { para } \alpha=\theta \text { ou } \nu ; \quad \phi^{\alpha}=b^{\prime}, \epsilon^{\alpha}=b, \quad \text { para } \alpha \neq \theta, \nu \\
& \eta^{(\theta \nu)}=c ; \quad \eta^{(\theta \alpha)}=\eta^{(\nu \alpha)}=d, \text { para } \alpha \neq \theta, \nu ; \quad \eta^{(\alpha \beta)}=e, \text { para } \alpha, \beta \neq \theta, \nu
\end{aligned}
$$

gera todo o espaço. Ortogonalidade com os vetores das demais classes implica: $a^{\prime}=b^{\prime}=$ $a=b=0 ; c=(2-n) d ; d=(3-n) e / 2$. O autovalor associado ao autovetor transversal acima é dado por:

$$
\lambda=P-2 Q+R
$$

de forma que a linha de estabilidade de Almeida e Thouless, caracterizada por $\lambda=0$, é obtida da equação:

$$
\left(\frac{k_{B} T}{J}\right)^{2}=(a+2 b x)^{2} \int_{-\infty}^{\infty} \frac{d z}{\sqrt{2 \pi}} \exp \left(-\frac{z^{2}}{2}\right) \operatorname{sech}^{4}\left[\beta(a+2 b x)\left(J q^{1 / 2} z+J_{0} m\right)\right]
$$

onde $q, m$ e $x$ são obtidos das condições de extremo (3.28), (3.29) e (3.30). 



\section{Apêndice B}

\section{Solução trigonométrica da equação}

\section{cúbica}

A equação (5.44) pode ser escrita na forma

$$
\lambda^{3}-\alpha \lambda^{2}-\beta=0,
$$

onde

$$
\alpha=2 \frac{\sigma}{T} \frac{1}{2-S}, \quad \beta=\frac{4+S}{2(2-S)}
$$

Podemos eliminar o termo quadrático por meio da substituição $\lambda=x-c$, escolhendo $c=-\alpha / 3$. Assim

$$
x^{3}+p x+q=0
$$

onde

$$
p=-\frac{\alpha^{2}}{3}, \quad q=-\left(\frac{2}{27} \alpha^{3}+\beta\right) .
$$

A solução trigonométrica explora a identidade

$$
\cosh 3 \theta=4 \cosh ^{3} \theta-3 \cosh \theta
$$

Se existir apenas uma raiz real, dado que $p<0$ e $q<0$, a substituição $x=u \cosh \theta$ em (B.3) resulta

$$
4 \cosh ^{3} \theta+4 \frac{p}{u^{2}} \cosh \theta+4 \frac{q}{u^{3}}=0
$$


ou ainda, escolhendo $u^{2}=-(4 / 3) p$,

$$
4 \cosh ^{3} \theta-3 \cosh \theta+\frac{4 q}{(-(4 / 3) p)^{3 / 2}}=0 .
$$

Usamos agora a identidade (B.5) para escrever

$$
\cosh 3 \theta=\frac{3}{2} \frac{q}{p} \sqrt{-\frac{3}{p}}
$$

ou seja

$$
\theta=\frac{1}{3} \operatorname{arccosh}\left(\frac{3}{2} \frac{q}{p} \sqrt{-\frac{3}{p}}\right)
$$

Logo

$$
x=u \cosh \theta=2 \sqrt{-\frac{p}{3}} \cosh \left[\frac{1}{3} \operatorname{arccosh}\left(\frac{3}{2} \frac{q}{p} \sqrt{-\frac{3}{p}}\right)\right],
$$

ou ainda, em termos das grandezas iniciais

$$
\lambda=\frac{2 \sigma}{3(2-S) T}\left\{1+2 \cosh \left[\frac{1}{3} \cosh ^{-1}\left(1+\frac{27(2-S)^{2}(4+S) T^{3}}{32 \sigma^{3}}\right)\right]\right\},
$$

o que demonstra a equação (5.46). 


\section{Referências Bibliográficas}

Acharya, B. R., Primak, A., e Kumar, S. (2004). Phys. Rev. Lett., 24, 145506.

Alben, R. (1973). J. Chem. Phys., 59, 4299.

Allahverdyan, A. E., e Nieuwenhuizen, T. M. (2000). Phys. Rev. E, 62, 845.

Allahverdyan, A. E., Nieuwenhuizen, T. M., e Saakian, D. B. (2000). Eur. Phys. J. B, 16,317 .

Allahverdyan, A. E., e Petrosyan, K. G. (2006). Phys. Rev. Lett., 96, 065701.

Allender, D., e Longa, L. (2008). Phys. Rev. E, 78, 011704.

Almeida, J. R. L. de, e Thouless, D. J. (1978). J. Phys. A: Math. Gen., 11, 983.

Apreutesei, D., e Mehl, G. H. (2006). Chem. Commun., 609.

Ashcroft, N. W., e Mermin, N. D. (1976). Solid state physics. Hold, Rinehart, and Winston, New York.

Barra, A., Guerra, F., e Mingione, E. (2011). arXiv:1104.2080v1.

Baxter, R. J. (1989). Exactly solved models in statistical mechanics. Academic Press, London.

Berardi, R., Muccioli, L., Orlandi, S., Ricci, M., e Zannoni, C. (2008). J. Phys.: Condens. Matter, 20, 463101.

Binder, K., e Reger, J. D. (1992). Adv. Phys., 41, 547.

Binder, K., e Young, A. P. (1986). Rev. Mod. Phys., 58, 801. 
Bouchaud, J., e Mézard, M. (1994). J. Phys. I (France), 4, 1109.

Brout, R. (1959). Phys. Rev., 115, 824.

Bruijn, N. G. (1981). Asymptotic methods in analysis. Dover, New York.

Brush, S. G. (1967). Rev. Mod. Phys., 39, 883.

Cannavacciuolo, L., e Landau, D. P. (2005). Phys. Rev. B, 71, 134104.

Cannella, V., e Mydosh, J. A. (1972). Phys. Rev. B, 6, 4220.

Cardy, J. (1996). Scaling and renormalization in statistical physics. Cambridge University Press, Cambridge.

Carmo, E. do, Liarte, D. B., e Salinas, S. R. (2010). Phys. Rev. E, 81, 062701.

Caticha, N. (1994). J. Phys. A: Math. Gen., 27, 5501.

Chandrasekhar, S. (1992). Liquid crystals (Second ed.). Cambridge University Press, Cambridge.

Chen, Z.-W., e Deutch, J. M. (1984). J. Chem. Phys., 80, 2151.

Chen, Z. Y., e Kardar, M. (1986). J. Phys. C: Solid State Phys., 19, 6825.

Clarke, S. M., Tajbakhsh, A. R., Terentjev, E. M., e Warner, M. (2001). Phys. Rev. Lett., 86, 4044.

Clarke, S. M., Terentjev, E. M., Kundler, I., e Finkelmann, H. (1998). Macromolecules, 31, 4862 .

Coolen, A. C. C., Penney, R. W., e Sherrington, D. (1993). J. Phys. A: Math. Gen., 26, 3681.

Courtens, E. (1983). Helv. Phys. Acta, 56, 705.

Cugliandolo, L. F., Kurchan, J., e Peliti, L. (1997). Phys. Rev. E, 55, 3898.

Date, R. W., e Bruce, D. W. (2003). J. Am. Chem. Soc., 125, 9012.

Domb, C. (1956). J. Chem. Phys., 25, 783. 
Dominicis, C. de, e Giardina, I. (2006). Random fields and spin glasses. Cambridge University Press, Cambridge.

Dorlas, T. C. (1999). Statistical mechanics: Fundamentals and model solutions. IOP Publishing, London.

Edwards, S. F., e Anderson, P. W. (1975). J. Phys. F-Metal Physics, 5, 965.

Ennis, R., Malacarne, L. C., Palffy-Muhoray, P., e Shelley, M. (2006). Phys. Rev. E, 74, 061802.

Feynman, R. P., Leighton, R. B., e Sands, M. (1963). The feynman lectures on physics (Vol. 1). Addison-Wesley Publishing Company, Massachusetts.

Figueiredo-Neto, A. M., e Salinas, S. R. A. (2005). The physics of lyotropic liquid crystals: Phase transitions and structural properties. Oxford University Press, Oxford.

Fischer, K. H., e Hertz, J. A. (1991). Spin glasses. Cambridge University Press, Cambridge.

Fisher, D. S., Grinstein, D. M., e Khurana, A. (1988). Physics Today, 41, 56.

Flory, P. (1956). Proc. R. Soc., A234, 73.

Flory, P. J. (1953). Principles of polymer chemistry. Cornell University Press, Ithaca.

Freiser, M. J. (1970). Phys. Rev. Lett., 24, 1041.

Fridrikh, S. V., e Terentjev, E. M. (1997). Phys. Rev. Lett., 79, 4661.

Fridrikh, S. V., e Terentjev, E. M. (1999). Phys. Rev. E, 60, 1847.

Fyodorov, Y. V., Korenblit, I. Y., e Shender, E. F. (1987). Europhys. Lett., 4, 827.

Gennes, P. G. de. (1975). C. R. Seances Acad. Sci. Ser. B, 281, 101.

Gennes, P. G. de, e Prost, J. (1993). The physics of liquid crystals. Claredon Press, Oxford.

Golubović, L., e Lubensky, T. C. (1989). Phys. Rev. Lett., 63, 1082. 
Gramsbergen, E. F., Longa, L., e Jeu, W. H. de. (1986). Phys. Rep., 135, 195.

Greinert, N., Wood, T., e Barlett, P. (2006). Phys. Rev. Lett., 97, 265702.

Guerra, F., e Toninelli, F. L. (2002). Commun. Math. Phys., 230, 71.

He, H., e Thorpe, M. F. (1985). Phys. Rev. Lett., 54, 2107.

Hemmen, J. L. van, e Palmer, R. G. (1979). J. Phys. A: Math. Gen., 12, 563.

Henriques, E. F., e Henriques, V. B. (1997). J. Chem. Phys., 107, 8036.

Henriques, V. B., e Salinas, S. R. (1987). J. Phys. C: Solid State Phys., 20, 2415.

Jayasri, D., Satyavathi, N., Sastry, V. S. S., e Murthy, K. P. N. (2009). Physica A, 388, 385.

Kadanoff, L. P. (2009). "More is the same; phase transitions and mean field theories". J. Stat. Phys.

Kirkpatrick, S., e Sherrington, D. (1978). Phys. Rev. B, 17, 4384.

Kittel, C. (1996). An introduction to solid state physics (Seventh ed.). John Willey \& Sons Inc., New York.

Korenblit, I. Y., e Shender, E. F. (1985). Sov. Phys.-JETP, 62, 1030.

Kouwer, P. H. J., e Mehl, G. H. (2003). J. Am. Chem. Soc., 125, 11172.

Kubo, R. (1988). Statistical mechanics: An advanced course with problems and solutions (Second ed.). Elsevier, Amsterdam.

Küpfer, J., e Finkelmann, H. (1991). Macromol. Chem. Rap. Commun., 12, 717.

Kurchan, J. (2005). Nature, 433, 222.

Landau, D. P. (2006). Braz. J. Phys., 36, 640.

Landau, L. D., Lifshitz, E. M., e Pitaevskii, L. P. (1980). Statistical physics (Third ed.). Pergamon Press, Oxford.

Lee, T. D., e Yang, C. N. (1952). Phys. Rev., 87, 410. 
Leuzzi, L. (2009). J. Non-Cryst. Solids, 355, 686.

Liarte, D. B., Salinas, S. R., e Yokoi, C. S. O. (2009). J. Phys. A: Math. Theor., 42, 205002.

Liarte, D. B., Salinas, S. R., e Yokoi, C. S. O. (2011). Phys. Rev. E, 84, 011124.

Liarte, D. B., e Yokoi, C. S. O. (2008). J. Phys. A: Math. Theor., 41, 324010.

Lu, B.-S., Ye, F., Xing, X., e Goldbart, P. M. (2011). e-print arXiv:1101.1323v1.

Luckhurst, G. R. (2004). Nature, 430, 413.

Madsen, L. A., Dingemans, T. J., Nakata, M., e Samulski, E. T. (2004). Phys. Rev. Lett., 92, 145505.

Maier, W., e Saupe, A. (1958). Z. Naturforsch. A: Phys. Sci., 13, 564.

Mamasakhlisov, Y. S., Naji, A., e Podgornik, R. (2008). J. Stat. Phys., 133, 659.

Marshall, A. H. (2007). Phys. Rev. B, 75, 054414.

Marshall, A. H., Chacraborty, B., e Nagel, S. (2006). Europhys. Lett., 74, 699.

Matsushita, E., e Matsubara, T. (1985). J. Phys. Soc. Japan, 54, 1161.

Mezard, M., Parisi, G., e Virasoro, M. (1987). Spin glass theory and beyond. World Scientific, Singapure.

Mitchell, S. J., Pereira, L. F. C., e Landau, D. P. (2008). Braz. J. Phys., 38, 1.

Nishimori, H. (2001). Statistical physics of spin glasses and information processing. Oxford University Press, Oxford.

Nogueira, E., Nobre, F. D., Costa, F. A. da, e Coutinho, S. (1998). Phys. Rev. E, 57, 5079.

Oliveira, M. J. de, e Neto, A. M. F. (1986). Phys. Rev. A, 34, 3481.

Onsager, L. (1944). Phys. Rev., 65, 117.

Onsager, L. (1949). Ann. N. Y. Acad. Sci., 51, 627. 
Ortiz, C., Ober, C. K., e Kramer, J. (1998). Polymer, 39, 3713.

Ortiz, C., Wagner, M., Bhargava, N., Ober, C. K., e Kramer, J. (1998). Macromolecules, $31,8531$.

Palffy-Muhoray, P., Bruyn, J. R. de, e Dunmur, D. A. (1985a). Mol. Cryst. Liq. Cryst., $127,301$.

Palffy-Muhoray, P., Bruyn, J. R. de, e Dunmur, D. A. (1985b). J. Chem. Phys., 82, 5294.

Papantopoulos, G., Papavassiliou, G., Milia, F., Schmidt, V. H., Drumheller, J. E., Pinto, N. J., Blinc, R., e Zalar, B. (1994). Phys. Rev. Lett., 73, 276.

Parisi, G. (1979). Physics Letters, 73A, 203.

Parisi, G. (1980a). J. Phys. A: Math. Gen., 13, L115.

Parisi, G. (1980b). J. Phys. A: Math. Gen., 13, 1101.

Parisi, G. (1980c). J. Phys. A: Math. Gen., 13, 1887.

Pasini, P., Skačej, G., e Zannoni, C. (2005). Chem. Phys. Lett., 413, 463.

Patel, D. M., e Fredrickson, G. H. (2003). Phys. Rev. E, 68, 051802.

Petridis, L., e Terentjev, E. M. (2006). Phys. Rev. E, 74, 051707.

Poderoso, F. C. (2007). Ecossistemas de replicadores: uma abordagem via mecânica estatística de sistemas desordenados. Tese de doutorado - IFSC-USP.

Poderoso, F. C., e Fontanari, J. F. (2007). J. Phys. A: Math. Theor., 40, 8723.

Reis, R. G. (2011). Transição de fase ordem-desordem em membranas na presença de dissociação: modelos estatísticos. Tese de doutorado de Renato Germano Reis Nunes - IFUSP.

Ritter, O. M., D'Ajello, P. C. T., e Figueiredo, W. (2004). Phys. Rev. E, 69, 016119.

Salinas, S. R. (1974). J. Phys. C: Solid State Phys., 7, 241. 
Salinas, S. R. (1997). Introdução à física estatística. Edusp, São Paulo.

Salinas, S. R., e Wreszinski, W. F. (1993). Disorder and competition in soluble lattice models. World Scientific, Singapore.

Selinger, J. V., Jeon, H. G., e Ratna, B. R. (2002). Phys. Rev. Lett., 89, 225701.

Selinger, J. V., e Ratna, B. R. (2004). Phys. Rev. E, 70, 041707.

Sharma, S. R., Palffy-Muhoray, P., Bergesersen, B., e Dunmur, D. A. (1985). Phys. Rev. A, 32, 3752 .

Sherrington, D. (2008). arXiv:cond-mat/0512425v2 [cond-mat.dis-nn].

Sherrington, D., e Kirkpatrick, S. (1975). Phys. Rev. Lett., 35, 1792.

Soares, R. F., Nobre, F. D., e Almeida, J. R. L. de. (1994). Phys. Rev. B, 50, 6151.

Tadic, B., Pirc, R., Blinc, R., Petersson, J., e Wiotte, W. (1994). Phys. Rev. B, 50, 9824 .

Talagrand, M. (2003). Spin glasses: A challenge for mathematicians. Springer, Berlin.

Thorpe, M. (1978). J. Phys. C: Solid State Phys., 11, 2983.

Uchida, N. (2000). Phys. Rev. E, 62, 5119.

Urayama, K., Kohmon, E., Kojima, M., e Takigawa, T. (2009). Macromolecules, 42, 4084 .

Verwey, G. C., Warner, M., e Terentjev, E. M. (1996). J. Phys. II France, 6, 1273.

Vieira, S. R., Nobre, F. D., e Yokoi, C. S. O. (2000). Phys. Rev. E, 61, 4760.

Warner, M., e Kutter, S. (2002). Phys. Rev. E, 65, 051707.

Warner, M., e Terentjev, E. M. (1996). Prog. Polym. Sci., 21, 853.

Warner, M., e Terentjev, E. M. (2003). Liquid crystal elastomers. Oxford University Press, Oxford.

Wu, F. Y., McCoy, B. M., Fisher, M., e Chayes, L. (2008). Phil. Mag., 88, 3093. 
Xing, X., Pfahl, S., Mukhopadhyay, S., Goldbart, P. M., e Zippelius, A. (2008). Phys. Rev. E, 77, 051802 .

Ye, F., e Lubensky, T. C. (2009). J. Phys. Chem. B, 113, 3853.

Yu, L. J., e Saupe, A. (1980). Phys. Rev. Lett., 45, 1000.

Yu, Y.-K., Taylor, P. L., e Terentjev, E. M. (1998). Phys. Rev. Lett., 81, 128.

Zhang, Z.-. D. (2007). Phil. Mag., 87, 5309.

Zwanzig, R. (1963). J. Chem. Phys., 39, 1714. 